\title{
Arithmetically Cohen-Macaulay bundles on cubic threefolds
}

\author{
Martí Lahoz, Emanuele Macrì and Paolo Stellari
}

\begin{abstract}
We study arithmetically Cohen-Macaulay bundles on cubic threefolds by using derived category techniques. We prove that the moduli space of stable Ulrich bundles of any rank is always non-empty by showing that it is birational to a moduli space of semistable torsion sheaves on the projective plane endowed with the action of a Clifford algebra. We describe this birational isomorphism via wall-crossing in the space of Bridgeland stability conditions, in the example of instanton sheaves of minimal charge.
\end{abstract}

\section{Introduction}

Fourier-Mukai techniques to study stable vector bundles on surfaces have been an extremely useful tool for more than 30 years. In this paper, we use a construction by Kuznetsov to generalize such a circle of ideas and study arithmetically Cohen-Macaulay (ACM) stable vector bundles on smooth projective cubic hypersurfaces. The basic idea is to use a semiorthogonal decomposition of the derived category of coherent sheaves to "reduce dimension". The disadvantage of this approach is that we have to consider complexes and a notion of stability for them; this forces us to restrict to the cubic threefold case (and to special examples in the fourfold case, treated in a forthcoming paper). The advantage is that this may lead to a general approach to study ACM stable bundles in higher dimensions.

\section{ACM bundles and semiorthogonal decompositions}

Let $Y \subset \mathbb{P}^{n+1}$ be a smooth complex cubic $n$-fold, and let $\mathcal{O}_{Y}(H)$ denote the corresponding very ample line bundle. A vector bundle $F$ on $Y$ is called arithmetically Cohen-Macaulay if $\operatorname{dim} H^{i}(Y, F(j H))=0$ for all $i=1, \ldots, n-1$ and all $j \in \mathbb{Z}$. In algebraic geometry, the interest in studying stable ACM bundles (and their moduli spaces) on projective varieties arose from the papers [Beau02, Dru00, Ili99, IM00, MT01]. In fact, in [Dru00] it is proved that the moduli space of rank two instanton sheaves on a cubic threefold is isomorphic to the blow-up of the intermediate Jacobian in (minus) the Fano surface of lines. The intermediate Jacobian can be used both to

Received 15 April 2013, accepted in final form 5 October 2014.

2010 Mathematics Subject Classification 18E30, 14E05

Keywords: arithmetically Cohen-Macaulay vector bundles, cubic threefolds

This journal is (C) Foundation Compositio Mathematica 2015. This article is distributed with Open Access under the terms of the Creative Commons Attribution Non-Commercial License, which permits non-commercial reuse, distribution, and reproduction in any medium, provided that the original work is properly cited. For commercial re-use, please contact the Foundation Compositio Mathematica.

M. L. is partially supported by SFB/TR 45, Fondation Mathématique Jacques Hadamard (FMJH) and MTM2012-38122-C03-02. E. M. is partially supported by the NSF grants DMS-1001482/DMS-1160466 and DMS1302730, the Hausdorff Center for Mathematics, Universität Bonn, and SFB/TR 45. P. S. is partially supported by the grants FIRB 2012 "Moduli Spaces and Their Applications" and the national research project "Geometria delle Varietà Proiettive" (PRIN 2010-11). 


\section{Martí Lahoz, Emanuele Macrì and PaOlo Stellari}

control the isomorphism type of the cubic, via the Clemens-Griffiths/Tyurin Torelli theorem, and to prove the non-rationality of the cubic (see [CG72]). From a more algebraic viewpoint, ACM bundles correspond to maximal Cohen-Macaulay (MCM) modules over the graded ring associated with the projectively embedded variety, and as such they have been extensively studied in the past years (see, for example, [Yos90]).

In a different direction, Kuznetsov studied in [Kuz04] semiorthogonal decompositions of the derived category of a cubic hypersurface. In fact, as we review in Section 1.1, there exists a nontrivial triangulated subcategory $\mathbf{T}_{Y} \subset \mathrm{D}^{\mathrm{b}}(Y)$ which might encode the birational information of the cubic. For example, in the case of a cubic threefold $Y$, it is proven in [BMMS12] that the isomorphism class of $Y$ can be recovered directly from $\mathbf{T}_{Y}$ as a sort of "categorical version" of the Clemens-Griffiths/Tyurin Torelli Theorem. In [Kuz10] it is conjectured that a cubic fourfold is rational if and only if the category $\mathbf{T}_{Y}$ is equivalent to the derived category of a K3 surface. For the interpretation of $\mathbf{T}_{Y}$ as a category of matrix factorization, we refer to [Orl09], while [BB13] deals with the interpretation as a summand of the Chow motive of Y.

For cubic threefolds, a different description of $\mathbf{T}_{Y}$ is available, via Kuznetsov's semiorthogonal decomposition of the derived category of a quadric fibration (see [Kuz08]). Indeed, as we review in Section 1.3, $\mathbf{T}_{Y}$ is equivalent to a full subcategory of the derived category of sheaves on $\mathbb{P}^{2}$ with the action of a sheaf of Clifford algebras $\mathcal{B}_{0}$ (determined by fixing a structure of quadric fibration on the cubic). We denote by $\Xi: \mathbf{T}_{Y} \hookrightarrow \mathrm{D}^{\mathrm{b}}\left(\mathbb{P}^{2}, \mathcal{B}_{0}\right)$ the induced fully faithful functor. The key observation (which is not surprising if we think of ACM bundles as MCM modules, see [CH11, Section 2] and [Orl09]) is the following: given a stable ACM bundle $F$ on $Y$, a certain twist of $F$ by the very ample line bundle $\mathcal{O}_{Y}(H)$ belongs to $\mathbf{T}_{Y}$ (this is Lemma 1.6). Hence, the idea is to study basic properties of ACM bundles on $Y$ (for example, existence and irreducibility of the moduli spaces) by using the functor $\Xi$, and so by considering them as complexes of $\mathcal{B}_{0}$-modules on $\mathbb{P}^{2}$. The principle is that, since $\mathrm{D}^{\mathrm{b}}\left(\mathbb{P}^{2}, \mathcal{B}_{0}\right)$ has dimension two, although it is not intrinsic to the cubic, it should still lead to several simplifications. The main question now becomes whether there exists a notion of stability for objects in $\mathrm{D}^{\mathrm{b}}\left(\mathbb{P}^{2}, \mathcal{B}_{0}\right)$ which corresponds to the usual stability for ACM bundles. In this paper we suggest that, for cubic threefolds, such a notion of stability in $\mathrm{D}^{\mathrm{b}}\left(\mathbb{P}^{2}, \mathcal{B}_{0}\right)$ should be Bridgeland stability [Bri07].

\section{Results}

Let $Y$ be a cubic threefold. By fixing a line $l_{0}$ in $Y$, the projection from $l_{0}$ to $\mathbb{P}^{2}$ gives a structure of a conic fibration on (a blow-up of) $Y$. The sheaf of algebras $\mathcal{B}_{0}$ on $\mathbb{P}^{2}$ mentioned before is nothing but the sheaf of even parts of the Clifford algebras associated with this conic fibration (see $\left[\right.$ Kuz10]). Denote by $\mathbf{C o h}\left(\mathbb{P}^{2}, \mathcal{B}_{0}\right)$ the abelian category of coherent $\mathcal{B}_{0}$-modules and by $\mathrm{D}^{\mathrm{b}}\left(\mathbb{P}^{2}, \mathcal{B}_{0}\right)$ the corresponding bounded derived category.

As a first step in the study of ACM bundles on $Y$, we consider the moduli spaces $\mathfrak{M}_{d}$ of Gieseker stable $\mathcal{B}_{0}$-modules in $\mathbf{C o h}\left(\mathbb{P}^{2}, \mathcal{B}_{0}\right)$ with Chern character $(0,2 d,-2 d)$, for any $d \geqslant 1$. These moduli spaces are tightly related to the geometry of $Y$ and the first general result we can prove is the following (see Theorem 2.12).

Theorem A. The moduli space $\mathfrak{M}_{d}$ is irreducible with a morphism $\Upsilon: \mathfrak{M}_{d} \rightarrow\left|\mathcal{O}_{\mathbb{P}^{2}}(d)\right|$ whose fiber over a general smooth curve $C$ in $\left|\mathcal{O}_{\mathbb{P}^{2}}(d)\right|$ is the disjoint union of $2^{5 d-1}$ copies of the Jacobian of $C$. Moreover, the stable locus $\mathfrak{M}_{d}^{s}$ is smooth of dimension $d^{2}+1$.

The geometry of $\mathfrak{M}_{1}$ and $\mathfrak{M}_{2}$ can be understood more explicitly. Indeed, it turns out that $\mathfrak{M}_{1}$ is the Fano variety of lines in $Y$ blown up at the line $l_{0}$ (see Proposition 2.13). On the 


\section{ACM BUNDLES ON CUBIC THREEFOLDS}

other hand, $\mathfrak{M}_{2}$ is a birational model of the intermediate Jacobian of $Y$ (see Theorem 3.10 for a more detailed statement). Both results are obtained via wall-crossing in the space of Bridgeland stability conditions on the triangulated category $\mathrm{D}^{\mathrm{b}}\left(\mathbb{P}^{2}, \mathcal{B}_{0}\right)$. Notice that such a wall-crossing depends on the choice of a line inside the cubic threefold. As a corollary, one gets that the moduli space of instanton sheaves on $Y$ (of charge two) is isomorphic to a moduli space of Bridgeland stable objects in $\mathrm{D}^{\mathrm{b}}\left(\mathbb{P}^{2}, \mathcal{B}_{0}\right)$ with prescribed Chern character (see Theorem 3.10).

As $\mathbf{T}_{Y}$ can be naturally identified with a full subcategory of $\mathrm{D}^{\mathrm{b}}\left(\mathbb{P}^{2}, \mathcal{B}_{0}\right)$, via the functor $\Xi$, one may want to consider objects of $\mathfrak{M}_{d}$ which are contained in $\mathbf{T}_{Y}$. These generically correspond to ACM bundles on $Y$. This way we can achieve the following theorem, which generalizes one of the main results in [CHGS12].

Theorem B. Let $Y$ be a cubic threefold. Then, for any $r \geqslant 2$, the moduli space of stable rank $r$ Ulrich bundles is non-empty and smooth of dimension $r^{2}+1$.

Recall that an Ulrich bundle $E$ is an ACM bundle whose graded module $\bigoplus_{m \in \mathbb{Z}} H^{0}(Y, E(m))$ has $3 \operatorname{rk}(E)$ generators in degree one (see Section 2.5 for a discussion about the chosen normalization). Compared to the first part of [CHGS12, Theorem 1.2], our result removes the genericity assumption.

We believe that Theorem A will also be useful in studying the irreducibility of the moduli space of stable Ulrich bundles. In fact, we expect the functor $\Xi$ to map all stable Ulrich bundles on $Y$ to Bridgeland stable objects in $\mathrm{D}^{\mathrm{b}}\left(\mathbb{P}^{2}, \mathcal{B}_{0}\right)$, thus generalizing Theorem 3.10 to the case $r>2$.

It is maybe worth pointing out that the proof of Theorem B, which is contained in Section 2.5, is based upon the same deformation argument as in [CHGS12]. The main difference is that, by using our categorical approach and the moduli spaces $\mathfrak{M}_{d}$, we can make it work also for small rank $(r=2,3)$. Indeed, the argument in [CHGS12] relies on the existence of an ACM curve on $Y$ of degree twelve and genus ten, proved by Geiß and Schreyer in the appendix to [CHGS12], only for a generic cubic threefold, using Macaulay2. Moreover, although we have focused on cubic threefolds, we believe that our approach might work for any quadric fibration. In particular, other interesting Fano threefolds of Picard rank one are the intersection of three quadrics in $\mathbb{P}^{6}$, the quartic hypersurface containing a double line, and the double covering of $\mathbb{P}^{3}$ ramified along a quartic with an ordinary double point (see [Beau77]).

\section{Related work}

The idea of using semiorthogonal decompositions to study ACM bundles by reducing dimension is influenced by [Kuz12]. More precisely, in loc. cit., Kuznetsov proposes to understand the geometry of moduli spaces of instanton bundles (of any charge) on cubic threefolds via the category $\mathrm{D}^{\mathrm{b}}\left(\mathbb{P}^{2}, \mathcal{B}_{0}\right)$ and the functor $\Xi$.

There have been many studies about ACM bundles of rank two in dimensions two and three. Besides the already mentioned results on instanton bundles on cubic threefolds, some papers in this direction are [AM09, BF09, CF09, CM05, Mad00]. The higher rank case has been investigated in [AG99, AM09, Mad05]. The papers [Mir10] and [PLT09] give a few examples of indecomposable ACM bundles of arbitrarily high rank. The already mentioned papers [CH11, CHGS12] contain a systematic study of stable ACM bundles in higher rank on cubic surfaces and threefolds. A general existence result for Ulrich bundles on hypersurfaces is given in [HUB91].

Regarding preservation of stability via the functor $\Xi$, the papers [BMMS12, MS12] study the case of ideal sheaves of lines on a cubic threefold. 


\section{Martí Lahoz, Emanuele Macrì and PaOlo Stellari}

\section{Plan of the paper}

This paper is organized as follows. Section 1 collects basic facts about semiorthogonal decompositions and general results about ACM bundles on cubic hypersurfaces. In particular, we show that stable ACM bundles are objects of $\mathbf{T}_{Y}$ (up to twists) and state a simple cohomological criterion for a coherent sheaf in $\mathbf{T}_{Y}$ to be ACM (see Lemmas 1.6 and 1.9). In Section 1.3 we review Kuznetsov's work on quadric fibrations.

Section 2 concerns the case of cubic threefolds where the first two results mentioned above are proved. The argument is based on a detailed description of the easiest case of $\mathfrak{M}_{1}$, which involves Bridgeland stability conditions (see Section 2.2). Some background on the latter subject is provided in the same section. In Sections 2.4 and 2.5 we prove Theorems A and B, respectively. The geometric applications to some simple wall-crossing phenomena are described in detail in Section 3, where we study the geometry of $\mathfrak{M}_{2}$ and its relation to instanton bundles.

\section{Notation}

Throughout this paper we work over the complex numbers. For a smooth projective variety $X$, we denote by $\mathrm{D}^{\mathrm{b}}(X)$ the bounded derived category of coherent sheaves on $X$. We refer to [Huy06] for basics on derived categories. If $X$ is not smooth, we denote by $X_{\text {reg }}$ the regular part of $X$. We set $\operatorname{hom}^{i}(-,-):=\operatorname{dim} \operatorname{Hom}^{i}(-,-)$, where $\operatorname{Hom}^{i}(-,-)$ is computed in an abelian or triangulated category which will be specified each time. This paper assumes some familiarity with basic constructions and definitions about moduli spaces of stable bundles. For example, we do not define explicitly the notion of slope and Gieseker stability, of Harder-Narasimhan (HN) and Jordan-Hölder (JH) factors of a (semistable) vector bundle. For this, we refer to [HL10]. The same book is our main reference for the standard construction of moduli spaces of stable sheaves. For the twisted versions of them we refer directly to [Sim94, Lie07].

In the following, we will use the short-hand notation (semi) stable to refer to stable (respectively, semistable). Gieseker stability will be simply called stability, while slope stability will be called $\mu$-stability.

\section{The derived category of a cubic hypersurface}

In this section we show that, on a smooth cubic hypersurface $Y$, all stable ACM bundles are well behaved with respect to Kuznetsov's semiorthogonal decomposition of the derived category. In particular, after recalling the notion of semiorthogonal decomposition of a derived category, we show that stable ACM bundles on $Y$ belong to the non-trivial component $\mathbf{T}_{Y}$ of $\mathrm{D}^{\mathrm{b}}(Y)$, up to twists by line bundles. We also introduce one of the basic tools for studying the derived category of cubic threefolds: Kuznetsov's description of the derived category of a quadric fibration.

\subsection{Semiorthogonal decompositions}

Let $X$ be a smooth projective variety, and let $\mathrm{D}^{\mathrm{b}}(X)$ be its bounded derived category of coherent sheaves.

Definition 1.1. A semiorthogonal decomposition of $\mathrm{D}^{\mathrm{b}}(X)$ is a sequence of full triangulated subcategories $\mathbf{T}_{1}, \ldots, \mathbf{T}_{m} \subseteq \mathrm{D}^{\mathrm{b}}(X)$ such that $\operatorname{Hom}_{\mathrm{D}^{\mathrm{b}}(X)}\left(\mathbf{T}_{i}, \mathbf{T}_{j}\right)=0$ for $i>j$, and for all $G \in \mathrm{D}^{\mathrm{b}}(X)$, there exists a chain of morphisms in $\mathrm{D}^{\mathrm{b}}(X)$

$$
0=G_{m} \rightarrow G_{m-1} \rightarrow \ldots \rightarrow G_{1} \rightarrow G_{0}=G
$$




\section{ACM BUNDLES ON CUBIC THREEFOLDS}

with cone $\left(G_{i} \rightarrow G_{i-1}\right) \in \mathbf{T}_{i}$ for all $i=1, \ldots, m$. We will denote such a decomposition by $\mathrm{D}^{\mathrm{b}}(X)=\left\langle\mathbf{T}_{1}, \ldots, \mathbf{T}_{m}\right\rangle$.

Definition 1.2. An object $F \in \mathrm{D}^{\mathrm{b}}(X)$ is exceptional if $\operatorname{Hom}_{\mathrm{D}^{\mathrm{b}}(X)}^{p}(F, F)=0$ for all $p \neq 0$, and $\operatorname{Hom}_{\mathrm{D}^{\mathrm{b}}(X)}(F, F) \cong \mathbb{C}$. A collection $\left\{F_{1}, \ldots, F_{m}\right\}$ of objects in $\mathrm{D}^{\mathrm{b}}(X)$ is called an exceptional collection if $F_{i}$ is an exceptional object for all $i$, and $\operatorname{Hom}_{\mathrm{D}^{\mathrm{b}}(X)}^{p}\left(F_{i}, F_{j}\right)=0$ for all $p$ and all $i>j$.

Remark 1.3. An exceptional collection $\left\{F_{1}, \ldots, F_{m}\right\}$ in $\mathrm{D}^{\mathrm{b}}(X)$ provides a semiorthogonal decomposition

$$
\mathrm{D}^{\mathrm{b}}(X)=\left\langle\mathbf{T}, F_{1}, \ldots, F_{m}\right\rangle,
$$

where, by abuse of notation, we denoted by $F_{i}$ the triangulated subcategory generated by $F_{i}$ (equivalent to the bounded derived category of finite dimensional vector spaces). Moreover,

$$
\mathbf{T}:=\left\langle F_{1}, \ldots, F_{m}\right\rangle^{\perp}=\left\{G \in \mathrm{D}^{\mathrm{b}}(X): \operatorname{Hom}^{p}\left(F_{i}, G\right)=0 \text { for all } p \text { and } i\right\} .
$$

Similarly, one can define ${ }^{\perp}\left\langle F_{1}, \ldots, F_{m}\right\rangle=\left\{G \in \mathbf{T}: \operatorname{Hom}^{p}\left(G, F_{i}\right)=0\right.$ for all $p$ and $\left.i\right\}$.

Let $F \in \mathrm{D}^{\mathrm{b}}(X)$ be an exceptional object. Consider the two functors, respectively left and right mutation, $\mathbf{L}_{F}, \mathbf{R}_{F}: \mathrm{D}^{\mathrm{b}}(X) \rightarrow \mathrm{D}^{\mathrm{b}}(X)$ defined by

$$
\begin{aligned}
\mathbf{L}_{F}(G) & :=\text { cone }(\mathrm{ev}: \operatorname{RHom}(F, G) \otimes F \rightarrow G) \\
\mathbf{R}_{F}(G) & :=\text { cone }\left(\mathrm{ev}^{\vee}: G \rightarrow \operatorname{RHom}(G, F)^{\vee} \otimes F\right)[-1],
\end{aligned}
$$

where $\operatorname{RHom}(-,-):=\oplus_{p} \operatorname{Hom}_{\mathrm{D}^{\mathrm{b}}(X)}^{p}(-,-)[-p]$. More intrinsically, let $\iota_{\perp}$ and $\iota_{F^{\perp}}$ be the full embeddings of ${ }^{\perp} F$ and $F^{\perp}$, respectively, into $\mathrm{D}^{\mathrm{b}}(X)$. Denote by $\iota_{\perp_{F}}^{*}$ and $\iota_{\perp_{F}}$ the left and right adjoints of $\iota_{\perp_{F}}$ and by $\iota_{F^{\perp}}^{*}$ and $\iota_{F^{\perp}}^{!}$the left and right adjoints of $\iota_{F^{\perp}}$, respectively. Then $\mathbf{L}_{F}=$ $\iota_{F^{\perp}} \circ \iota_{F \perp}^{*}$, while $\mathbf{R}_{F}=\iota_{\perp F} \circ \iota_{\perp_{F}}$ (see, for example, [Kuz07, Section 2]).

The main property of mutations is that, given a semiorthogonal decomposition of $\mathrm{D}^{\mathrm{b}}(X)$

$$
\left\langle\mathbf{T}_{1}, \ldots, \mathbf{T}_{k}, F, \mathbf{T}_{k+1}, \ldots, \mathbf{T}_{n}\right\rangle,
$$

we can produce two new semiorthogonal decompositions

$$
\left\langle\mathbf{T}_{1}, \ldots, \mathbf{T}_{k}, \mathbf{L}_{F}\left(\mathbf{T}_{k+1}\right), F, \mathbf{T}_{k+2}, \ldots, \mathbf{T}_{n}\right\rangle
$$

and

$$
\left\langle\mathbf{T}_{1}, \ldots, \mathbf{T}_{k-1}, F, \mathbf{R}_{F}\left(\mathbf{T}_{k}\right), \mathbf{T}_{k+1}, \ldots, \mathbf{T}_{n}\right\rangle .
$$

Let us specify the relation between left and right mutations that will be used throughout this paper. Denote by $S_{X}=(-) \otimes \omega_{X}[\operatorname{dim}(X)]$ the Serre functor of $X$. We have the following lemma (which actually works more generally for any admissible subcategory in $\mathrm{D}^{\mathrm{b}}(X)$ ).

Lemma 1.4. If $F$ is an exceptional object, then $\mathbf{R}_{S_{X}(F)}$ is right adjoint to $\mathbf{L}_{F}$ while $\mathbf{R}_{F}$ is left adjoint to $\mathbf{L}_{F}$.

Proof. This follows from the remark that ${ }^{\perp}\left(S_{X}(F)\right)=F^{\perp}$ and by using adjunction between the functors $\iota_{\mathbf{D}}^{*}, \iota_{\mathbf{D}}$, and $\iota_{\mathbf{D}}^{!}$for $\mathbf{D}$ equal to ${ }^{\perp} F$ or to $F^{\perp}$.

\subsection{ACM bundles on cubics}

Let $Y$ be a smooth cubic $n$-fold, namely a smooth projective hypersurface of degree three in $\mathbb{P}^{n+1}$. We set $\mathcal{O}_{Y}(H):=\left.\mathcal{O}_{\mathbb{P}^{n+1}}(H)\right|_{Y}$. According to Remark 1.3, as observed by Kuznetsov, the 


\section{Martí Lahoz, Emanuele Macrì and PaOlo Stellari}

derived category $\mathrm{D}^{\mathrm{b}}(Y)$ of coherent sheaves on $Y$ has a semiorthogonal decomposition

$$
\mathrm{D}^{\mathrm{b}}(Y)=\left\langle\mathbf{T}_{Y}, \mathcal{O}_{Y}, \mathcal{O}_{Y}(H), \ldots, \mathcal{O}_{Y}((n-2) H)\right\rangle,
$$

where, by definition,

$$
\begin{aligned}
\mathbf{T}_{Y} & :=\left\langle\mathcal{O}_{Y}, \ldots, \mathcal{O}_{Y}(n-2)\right\rangle^{\perp} \\
& =\left\{G \in \mathrm{D}^{\mathrm{b}}(Y): \operatorname{Hom}_{\mathrm{D}^{\mathrm{b}}(Y)}^{p}\left(\mathcal{O}_{Y}(i H), G\right)=0 \text { for all } p \text { and } i=0, \ldots, n-2\right\} .
\end{aligned}
$$

Let us first recall the following definition.

Definition 1.5. (i) A vector bundle $F$ on a smooth projective variety $X$ of dimension $n$ is arithmetically Cohen-Macaulay (ACM) if $\operatorname{dim} H^{i}(X, F(j H))=0$ for all $i=1, \ldots, n-1$ and all $j \in \mathbb{Z}$.

(ii) An ACM bundle $F$ is called balanced if $\mu(F) \in[-1,0)$.

The following lemmas show that the category $\mathbf{T}_{Y}$ and stable ACM bundles are closely related.

LEMmA 1.6. Let $Y \subset \mathbb{P}^{n+1}$ be a smooth cubic $n$-fold. Let $F$ be a balanced $\mu$-stable ACM bundle with $\operatorname{rk}(F)>1$. Then $F \in \mathbf{T}_{Y}$.

Proof. We want to show that $h^{i}(Y, F(-j H))=0$ for all $i \in \mathbb{Z}$ and $j \in\{0, \ldots, n-2\}$. Since $F$ is ACM, we already have $h^{i}(Y, F(-j H))=0$ for $i \in\{1, \ldots, n-1\}$ and any $j$. Hence, we only need to prove that $h^{0}(Y, F(-j H))=h^{n}(Y, F(-j H))=0$ for $j \in\{0, \ldots, n-2\}$. But, on the one hand, we have

$$
h^{0}(Y, F(-j H))=\operatorname{hom}\left(\mathcal{O}_{Y}(j H), F\right)=0
$$

for $j \geqslant 0$, since $F$ is $\mu$-semistable with $\mu(F)<0$. On the other hand,

$$
h^{n}(Y, F(-j H))=\operatorname{ext}^{n}\left(\mathcal{O}_{Y}(j H), F\right)=\operatorname{hom}\left(F, \mathcal{O}_{Y}((-n+1+j) H)\right)=0
$$

for $-n+1+j<-1$, because $F$ is $\mu$-semistable with $-1 \leqslant \mu(F)$. It remains to prove that the vector space $\operatorname{Hom}\left(F, \mathcal{O}_{Y}((-n+1+j) H)\right)$ is trivial for $j=n-2$. But this is immediate, since $F$ is a $\mu$-stable sheaf of rank greater than one.

Remark 1.7. The previous lemma can be generalized slightly. Indeed, the same proof works for a balanced ACM bundle of rank greater than one, if it is $\mu$-semistable and $\operatorname{Hom}\left(F, \mathcal{O}_{Y}(-H)\right)=0$.

Remark 1.8. When $n=4$, the Serre functor of the subcategory $\mathbf{T}_{Y}$ is isomorphic to the shift by two (see [Kuz10, Theorem 4.3]). Thus, as an application of the result above and [KM09, Theorem 4.3], one gets that the smooth locus of any moduli space of $\mu$-stable ACM vector bundles on $Y$ carries a closed symplectic form.

Lemma 1.9. Let $Y \subset \mathbb{P}^{n+1}$ be a smooth cubic $n$-fold and let $F \in \mathbf{C o h}(Y) \cap \mathbf{T}_{Y}$. Assume

$$
\begin{aligned}
& H^{1}(Y, F(H))=0, \\
& H^{1}(Y, F((1-n) H))=\ldots=H^{n-1}(Y, F((1-n) H))=0 .
\end{aligned}
$$

Then $F$ is an ACM bundle.

Proof. We start by proving that $H^{i}(Y, F(j H))=0$ for all $i=1, \ldots, n-1$ and all $j \in \mathbb{Z}$. Denote by $i: Y \hookrightarrow \mathbb{P}^{n+1}$ the embedding of $Y$. For $m \in \mathbb{Z}$, we recall the Beilinson spectral sequence from [Huy06, Proposition 8.28]:

$$
E_{1}^{p, q}:=H^{q}\left(\mathbb{P}^{n+1}, i_{*} F(p+m)\right) \otimes \Omega_{\mathbb{P}^{n+1}}^{-p}(-p) \Rightarrow E^{p+q}= \begin{cases}F(m) & \text { if } p+q=0 \\ 0 & \text { otherwise }\end{cases}
$$




\section{ACM BUNDLES ON CUBIC THREEFOLDS}

We first consider the case when $m=1$. Since $F \in \mathbf{T}_{Y}$, we have $E_{1}^{p, q}=0$ for $p=-n+1, \ldots,-1$ and all $q$. By assumption, $E_{1}^{p, q}=0$, also for $p=-n$ and $q=1, \ldots, n-1$, and $E_{1}^{0,1}=0$. As a consequence, all the differentials $d_{r}^{-n-1, q}$ are 0 for all $q=1, \ldots, n-1$ and all $r>0$. Hence, $E_{1}^{-n-1, q}=0$ for all $q=1, \ldots, n-1$. A similar argument shows that $E_{1}^{0, q}=0$ for all $q=1, \ldots, n$. Summing up, we have the following vanishing:

$$
\begin{array}{ll}
H^{i}(Y, F(j H))=0 & \text { for all } i=1, \ldots, n-1 \text { and all } j=-n, \ldots, 0,1, \\
H^{0}(Y, F(j H))=0 & \text { for all } j \leqslant 0 \\
H^{n}(Y, F(j H))=0 & \text { for all } j \geqslant-n+2 .
\end{array}
$$

Now, on the one hand, by using the Beilinson spectral sequence for $m>1$ and the vanishing (1.2.3) and (1.2.4), we can prove by induction on $m$ that

$$
H^{i}(Y, F(j H))=0 \quad \text { for all } i=1, \ldots, n-1 \text { and all } j \leqslant 0 .
$$

On the other hand, for $m<1$, the vanishing (1.2.3) and (1.2.5) show

$$
H^{i}(Y, F(j H))=0 \quad \text { for all } i=1, \ldots, n-1 \text { and all } j>0 .
$$

To finish the proof of the lemma, we only need to show that $F$ is locally-free. Hence, it is enough to prove that $\mathcal{E} x t^{i}\left(F, \mathcal{O}_{Y}\right)=0$ for all $i>0$. For $k \in \mathbb{Z}$, consider the local-to-global spectral sequence

$$
E_{2}^{p, q}(k)=H^{p}\left(Y, \mathcal{E} x t^{q}\left(F(k), \mathcal{O}_{Y}\right)\right) \Rightarrow \operatorname{Ext}^{p+q}\left(F(k), \mathcal{O}_{Y}\right) .
$$

Assume, for a contradiction, that $\mathcal{E} x t^{i}\left(F, \mathcal{O}_{Y}\right) \neq 0$ for some $i>0$. Then, for $k \ll 0, E_{2}^{0, i}(k) \neq 0$, while $E_{2}^{p, i}(k)=0$ for $p>0$. From the spectral sequence and Serre duality, we deduce that $H^{n-i}(Y, F((k-n+1) H)) \neq 0$ for $i=1, \ldots, n$ and for $k \ll 0$, contradicting (1.2.6) and (1.2.4).

Finally, for later use, we recall how to construct autoequivalences of $\mathbf{T}_{Y}$ (not fixing the intersection $\left.\operatorname{Coh}(Y) \cap \mathbf{T}_{Y}\right)$.

Lemma 1.10. Let $Y \subset \mathbb{P}^{n+1}$ be a smooth cubic $n$-fold. Then, the functor

$$
\Theta: \mathbf{T}_{Y} \rightarrow \mathbf{T}_{Y}, \quad F \mapsto \mathbf{L}_{\mathcal{O}_{Y}}\left(F \otimes \mathcal{O}_{Y}(H)\right)
$$

is an autoequivalence of $\mathbf{T}_{Y}$.

Proof. Clearly $\mathbf{L}_{\mathcal{O}_{Y}}\left(F \otimes \mathcal{O}_{Y}(H)\right)$ belongs to $\mathbf{T}_{Y}$, and the inverse of $\Theta$ is given by the exact functor $\Theta^{-1}(-):=\mathcal{O}_{Y}(-H) \otimes \mathbf{R}_{\mathcal{O}_{Y}}(-)$.

Let us revise a classical example under a slightly different perspective.

EXAmple 1.11. Denote by $\mathfrak{M}_{Y}(v)$ the moduli space of $\mu$-stable torsion-free sheaves $E$ on $Y$ with $v:=\operatorname{ch}(E)=(2,-H,-l / 2,1 / 2)$, and let $F(Y)$ be the Fano surface of lines contained in a cubic threefold $Y$. In [BBR08, Theorem 1] it is proven that there exists a connected component $\mathfrak{M}_{Y}^{\prime}(v) \subset \mathfrak{M}_{Y}(v)$ consisting of $\mu$-stable ACM bundles such that $\mathfrak{M}_{Y}^{\prime}(v) \cong F(Y)$.

This can be obtained by using a slightly different approach. First of all, observe that the ideal sheaves of lines $\mathcal{I}_{l}$ is an element of $\mathbf{T}_{Y}$ for all $l \subset Y$ and that $F(Y)$ is the moduli space of these sheaves. By applying $\Theta[-1]$ (see Lemma 1.10), we get an exact sequence in $\operatorname{Coh}(Y)$

$$
0 \rightarrow F_{l}:=\Theta\left(\mathcal{I}_{l}\right)[-1] \rightarrow \mathcal{O}_{Y} \otimes_{\mathbb{C}} H^{0}\left(Y, \mathcal{I}_{l}(H)\right) \rightarrow \mathcal{I}_{l}(H) \rightarrow 0 .
$$

In particular, all $F_{l}$ are torsion-free sheaves with Chern character $v$. By (1.2.8) and Lemma 1.9, we deduce that $F_{l}$ are all ACM bundles. Since they belong to $\mathbf{T}_{Y}$, we have $H^{0}\left(Y, F_{l}\right)=0$ and as 


\section{Martí Lahoz, Emanuele Macrì and PaOlo Stellari}

they have rank two, this shows that they are $\mu$-stable. By construction, the Fano variety of lines is then a connected component of $\mathfrak{M}_{Y}(v)$.

By [BBR08, Lemma 1], the connected component $\mathfrak{M}_{Y}^{\prime}(v)$ can also be characterized as the component of $\mathfrak{M}_{Y}(v)$ consisting of $\mu$-stable torsion-free sheaves $G$ satisfying $H^{0}(Y, G(H)) \neq 0$ and $H^{0}(Y, G)=0$. Also, by Lemma 1.6 and by [BMMS12, Theorem 4.1 and Proposition 4.2], all balanced $\mu$-stable ACM bundles $G$ with $\operatorname{Ext}^{1}(G, G) \cong \mathbb{C}^{2}$ are in $\mathfrak{M}_{Y}^{\prime}(v)$.

Remark 1.12. The original proof in [BBR08] of the result in Example 1.11 relies on the so called Serre's construction which we briefly recall in a more general form (see, for example, [Arr07]). Let $X$ be a smooth projective manifold of dimension at least three and let $E$ be a rank $r$ vector bundle on $X$ which is spanned by its global sections. The dependency locus of $r-1$ general sections $s_{1}, \ldots, s_{r-1}$ of $E$ is a locally complete intersection subscheme $V$ of codimension two in $X$. If $L=\operatorname{det}(E)$, then the twisted canonical bundle $K_{V} \otimes L^{-1}$ is generated by $r-1$ sections.

Conversely, let $V$ a codimension two locally complete intersection subscheme of $X$ and let $L$ be a line bundle on $X$ such that $H^{2}\left(X, L^{-1}\right)=0$. If $K_{V} \otimes L^{-1}$ is generated by $r-1$ global sections, then $V$ can be obtained as the dependency locus of $r-1$ sections of $E$.

This construction is ubiquitous in the literature and it has been extensively used in various papers to produce examples of stable ACM bundles.

\subsection{Quadric fibrations}

The results of [Kuz08] on the structure of the derived category of coherent sheaves on a fibration in quadrics will be the basic tools to study the derived category of cubic threefolds. We briefly summarize them here.

Consider a smooth algebraic variety $S$ and a vector bundle $E$ of rank $n$ on $S$. We consider the projectivization $q: \mathbb{P}_{S}(E) \rightarrow S$ of $E$ on $S$ endowed with the line bundle $\mathcal{O}_{\mathbb{P}_{S}(E) / S}(1)$. Given a line bundle $L$ on $S$ and an inclusion of vector bundles $\sigma: L \rightarrow \operatorname{Sym}^{2} E^{\vee}$, we denote by $\alpha: X \hookrightarrow \mathbb{P}_{S}(E)$ the zero locus of $\sigma$ and by $\pi: X \rightarrow S$ the restriction of $q$ to $X$. It is not difficult to prove that $\pi$ is a flat quadric fibration of relative dimension $n-2$. The geometric picture can be summarized by the following diagram:

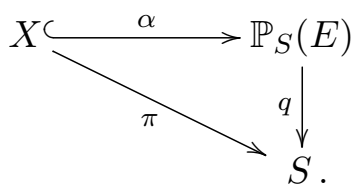

The quadric fibration $\pi: X \rightarrow S$ carries a sheaf $\mathcal{B}_{\sigma}$ of Clifford algebras. In fact, $\mathcal{B}_{\sigma}$ is the relative sheafified version of the classical Clifford algebra associated with a quadric on a vector space (more details can be found in [Kuz08, Section 3]). As in the absolute case, $\mathcal{B}_{\sigma}$ has an even part $\mathcal{B}_{0}$ whose description as an $\mathcal{O}_{S}$-module is as follows:

$$
\mathcal{B}_{0} \cong \mathcal{O}_{S} \oplus\left(\wedge^{2} E \otimes L\right) \oplus\left(\wedge^{4} E \otimes L^{2}\right) \oplus \ldots
$$

The odd part $\mathcal{B}_{1}$ of $\mathcal{B}_{\sigma}$ is such that

$$
\mathcal{B}_{1} \cong E \oplus\left(\wedge^{3} E \otimes L\right) \oplus\left(\wedge^{5} E \otimes L^{2}\right) \oplus \ldots
$$

We also write $\mathcal{B}_{2 i}=\mathcal{B}_{0} \otimes L^{-i}$ and $\mathcal{B}_{2 i+1}=\mathcal{B}_{1} \otimes L^{-i}$.

We write $\operatorname{Coh}\left(S, \mathcal{B}_{0}\right)$ for the abelian category of coherent $\mathcal{B}_{0}$-modules on $S$ and $\mathrm{D}^{\mathrm{b}}\left(S, \mathcal{B}_{0}\right)$ for its derived category. 


\section{ACM BUNDLES ON CUBIC THREEFOLDS}

Theorem 1.13 ([Kuz08, Theorem 4.2]). If $\pi: X \rightarrow S$ is a quadric fibration as above, then there exists a semiorthogonal decomposition

$\mathrm{D}^{\mathrm{b}}(X)=\left\langle\mathrm{D}^{\mathrm{b}}\left(S, \mathcal{B}_{0}\right), \pi^{*}\left(\mathrm{D}^{\mathrm{b}}(S)\right) \otimes \mathcal{O}_{X / S}(1), \pi^{*}\left(\mathrm{D}^{\mathrm{b}}(S)\right) \otimes \mathcal{O}_{X / S}(2), \ldots, \pi^{*}\left(\mathrm{D}^{\mathrm{b}}(S)\right) \otimes \mathcal{O}_{X / S}(n-2)\right\rangle$, where $\mathrm{D}^{\mathrm{b}}\left(S, \mathcal{B}_{0}\right)$ is the derived category of coherent sheaves of $\mathcal{B}_{0}$-modules on $S$.

In order to make this result precise, we need to give the definition of the fully faithful functor $\mathrm{D}^{\mathrm{b}}\left(S, \mathcal{B}_{0}\right) \rightarrow \mathrm{D}^{\mathrm{b}}(X)$ providing the embedding in the semiorthogonal decomposition above. The exact functor $\Phi:=\Phi_{\mathcal{E}^{\prime}}: \mathrm{D}^{\mathrm{b}}\left(S, \mathcal{B}_{0}\right) \rightarrow \mathrm{D}^{\mathrm{b}}(X)$ is defined as the Fourier-Mukai transform

$$
\Phi_{\mathcal{E}^{\prime}}(-):=\pi^{*}(-) \otimes_{\pi^{*} \mathcal{B}_{0}} \mathcal{E}^{\prime},
$$

where $\mathcal{E}^{\prime} \in \mathbf{C o h}(X)$ is a rank $2^{n-2}$ vector bundle on $X$ with a natural structure of flat left $\pi^{*} \mathcal{B}_{0}$-module defined by the short exact sequence

$$
0 \longrightarrow q^{*} \mathcal{B}_{0} \otimes \mathcal{O}_{\mathbb{P}_{S}(E) / S}(-2) \longrightarrow q^{*} \mathcal{B}_{1} \otimes \mathcal{O}_{\mathbb{P}_{S}(E) / S}(-1) \longrightarrow \alpha_{*} \mathcal{E}^{\prime} \longrightarrow 0 .
$$

In the notation of [Kuz08, Lemma 4.5], $\mathcal{E}^{\prime}=\mathcal{E}_{-1,1}^{\prime}$. The left adjoint functor of $\Phi$ is

$$
\Psi(-):=\pi_{*}\left((-) \otimes_{\mathcal{O}_{X}} \mathcal{E} \otimes_{\mathcal{O}_{X}} \operatorname{det} E^{\vee}[n-2]\right),
$$

where $\mathcal{E} \in \mathbf{C o h}(X)$ is another rank $2^{n-2}$ vector bundle with a natural structure of right $\pi^{*} \mathcal{B}_{0^{-}}$ module (see again [Kuz08, Section 4]). The analogous presentation of $\mathcal{E}$ is

$$
0 \longrightarrow q^{*} \mathcal{B}_{-1} \otimes \mathcal{O}_{\mathbb{P}_{S}(E) / S}(-2) \longrightarrow q^{*} \mathcal{B}_{0} \otimes \mathcal{O}_{\mathbb{P}_{S}(E) / S}(-1) \longrightarrow \alpha_{*} \mathcal{E} \longrightarrow 0
$$

In the notation of [Kuz08, Lemma 4.5$], \mathcal{E}=\mathcal{E}_{-1,0}$.

The category of $\mathcal{B}_{0}$-modules may be hard to work with directly. In some cases, we can reduce to a category of modules over a sheaf of Azumaya algebras, which is easier to deal with. We conclude this section by recalling this interpretation (see [Kuz08, Sections 3.5 and 3.6]). We define $S_{1} \subset S$ to be the degeneracy locus of $\pi$, namely the subscheme parametrizing singular quadrics, and $S_{2} \subset S_{1}$ to be the locus of singular quadrics of corank at least two. There are two separate cases to consider, according to parity of $n$.

In this paper we just need to study the case when $n$ is odd. To this end, let $f: \widehat{S} \rightarrow S$ be the stack of second roots of $\mathcal{O}_{S}\left(S_{1}\right)$ along the section $S_{1}$. An object of this stack over $T \rightarrow S$ is a triple $(L, \phi, \delta)$, where $L$ is a line bundle over $T, \phi$ is an isomorphism of $L^{2}$ with the pullback of $\mathcal{O}_{S}\left(S_{1}\right)$ to $T$, and $\delta$ is a section of $L$ such that $\phi\left(\delta^{2}\right)=S_{1}$ (see [AGV08, Cad07]). Locally over $S$, the category of coherent sheaves on $\widehat{S}$ can be identified with the category of coherent sheaves on the double covering of $S$ ramified along $S_{1}$ which are $\mathbb{Z} / 2 \mathbb{Z}$-equivariant with respect to the involution of the double covering (which only exists locally), that is, the category of coherent sheaves on the quotient stack of the double cover by the involution. Kuznestov calls the noncommutative variety $\widehat{S}$, "S with a $\mathbb{Z} / 2 \mathbb{Z}$-stack structure along $S_{1}$ " (see [Kuz08, Example 2.2]).

Proposition 1.14 ([Kuz08, Proposition 3.15]). There exists a sheaf of algebras $\mathcal{A}_{0}$ on $\widehat{S}$ such that $f_{*} \mathcal{A}_{0}=\mathcal{B}_{0}$ and that

$$
f_{*}: \operatorname{Coh}\left(\widehat{S}, \mathcal{A}_{0}\right) \stackrel{\sim}{\rightarrow} \operatorname{Coh}\left(S, \mathcal{B}_{0}\right)
$$

is an equivalence of categories. Moreover, the restriction of $\mathcal{A}_{0}$ to the complement of $\widehat{S}_{2}=f^{-1}\left(S_{2}\right)$ in $\widehat{S}$ is a sheaf of Azumaya algebras.

This will be the case for any cubic threefold. In fact, since we have assumed from the beginning that a cubic threefold is smooth and that the projection line is generic, $S_{1}$ is smooth and $S_{2}$ is empty. 


\section{Martí Lahoz, Emanuele Macrì and PaOlo Stellari}

\section{Cubic threefolds}

This section contains the proofs of our main results on ACM bundles on cubic threefolds. The goal is to generalize a result of Casanellas-Hartshorne on Ulrich bundles.

As explained in the introduction, the idea is to use Kuznetsov's results on quadric fibrations to reduce the problem of studying ACM bundles on a cubic threefold to the study of complexes of sheaves on $\mathbb{P}^{2}$ with the action of a sheaf of Clifford algebras $\mathcal{B}_{0}$.

The main technical parts are Sections 2.2 and 2.3; there we prove some results on moduli spaces of objects in $\mathrm{D}^{\mathrm{b}}\left(\mathbb{P}^{2}, \mathcal{B}_{0}\right)$ which are stable with respect to a Bridgeland stability condition. We come back to Ulrich bundles on cubic threefolds in Section 2.5.

\subsection{The setting}

Let $Y \subset \mathbb{P}^{4}$ be a cubic threefold. Let $l_{0} \subseteq Y$ be a general line and consider the blow-up $\widetilde{\mathbb{P}}$ of $\mathbb{P}^{4}$ along $l_{0}$. By "general" we mean that, if $l$ is any other line meeting $l_{0}$, then the plane containing them intersects the cubic in three distinct lines (we just avoid the lines of second type, see [CG72, Definition 6.6]). We set $q: \widetilde{\mathbb{P}} \rightarrow \mathbb{P}^{2}$ to be the $\mathbb{P}^{2}$-bundle induced by the projection from $l_{0}$ onto a plane and we denote by $\widetilde{Y}$ the strict transform of $Y$ via this blow-up. The restriction of $q$ to $\widetilde{Y}$ induces a conic fibration $\pi: \widetilde{Y} \rightarrow \mathbb{P}^{2}$. The geometric picture can be summarized by the following diagram:

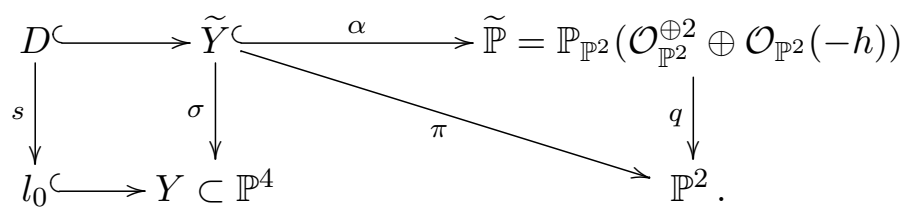

In particular, the vector bundle $E$ on $S=\mathbb{P}^{2}$ introduced in Section 1.3 is now $\mathcal{O}_{\mathbb{P}^{2}}^{\oplus 2} \oplus \mathcal{O}_{\mathbb{P}^{2}}(-h)$. Set $D \subset \widetilde{Y}$ to be the exceptional divisor of the blow-up $\sigma: \widetilde{Y} \rightarrow Y$. We denote by $h$ both the class of a line in $\mathbb{P}^{2}$ and its pull-backs to $\widetilde{\mathbb{P}}$ and $\widetilde{Y}$. We call $H$ both the class of a hyperplane in $\mathbb{P}^{4}$ and its pull-backs to $Y, \widetilde{\mathbb{P}}$, and $\widetilde{Y}$. We recall that $\mathcal{O}_{\widetilde{Y}}(D) \cong \mathcal{O}_{\widetilde{Y}}(H-h)$, the relative ample line bundle is $\mathcal{O}_{\widetilde{\mathbb{P}}}(H)$, the relative canonical bundle is $\mathcal{O}_{\widetilde{\mathbb{P}}}(h-3 H)$, and the dualizing line bundle $\omega_{\widetilde{Y}}$ is isomorphic to $\mathcal{O}_{\widetilde{Y}}(-h-H)$ (see, for example, [Kuz10, Lemma 4.1]).

The sheaf of even (respectively, odd) parts of the Clifford algebra corresponding to $\pi$, from Section 1.3, specializes in the case of cubic threefolds to

$$
\begin{aligned}
& \mathcal{B}_{0} \cong \mathcal{O}_{\mathbb{P}^{2}} \oplus \mathcal{O}_{\mathbb{P}^{2}}(-h) \oplus \mathcal{O}_{\mathbb{P}^{2}}(-2 h)^{\oplus 2}, \\
& \mathcal{B}_{1} \cong \mathcal{O}_{\mathbb{P}^{2}}^{\oplus 2} \oplus \mathcal{O}_{\mathbb{P}^{2}}(-h) \oplus \mathcal{O}_{\mathbb{P}^{2}}(-2 h),
\end{aligned}
$$

as sheaves of $\mathcal{O}_{\mathbb{P}^{2}}$-modules. The rank two vector bundles $\mathcal{E}^{\prime}$ and $\mathcal{E}$ sit in the short exact sequences provided by (1.3.1) and (1.3.3), respectively, where $L=\mathcal{O}_{\mathbb{P}^{2}}(-h)$.

Following [Kuz10] and [BMMS12, Section 2.1], one can give a description of the full subcategory $\mathbf{T}_{Y}$ in the semiorthogonal decomposition (1.2.1) of $\mathrm{D}^{\mathrm{b}}(Y)$. Indeed, first consider the semiorthogonal decomposition of $\mathrm{D}^{\mathrm{b}}(\widetilde{Y})$ in Theorem 1.13 and the one

$$
\mathrm{D}^{\mathrm{b}}(\tilde{Y})=\left\langle\sigma^{*}\left(\mathbf{T}_{Y}\right), \mathcal{O}_{\tilde{Y}}, \mathcal{O}_{\widetilde{Y}}(H), i_{*} \mathcal{O}_{D}, i_{*} \mathcal{O}_{D}(H)\right\rangle
$$

obtained by thinking of $\widetilde{Y}$ as the blow-up of $Y$ along $l_{0}$ and using the main result in [Or193]. Then one shows that

$$
\mathbf{R}_{\mathcal{O}_{\tilde{Y}}(-h)} \circ \Phi\left(\mathrm{D}^{\mathrm{b}}\left(\mathbb{P}^{2}, \mathcal{B}_{0}\right)\right)=\left\langle\sigma^{*} \mathbf{T}_{Y}, \mathcal{O}_{\widetilde{Y}}(h-H)\right\rangle
$$




\section{ACM BUNDLES ON CUBIC THREEFOLDS}

and thus we get a fully faithful embedding

$$
\Xi_{3}:=\left(\sigma_{*} \circ \mathbf{R}_{\mathcal{O}_{\tilde{Y}}(-h)} \circ \Phi\right)^{-1}: \mathbf{T}_{Y} \rightarrow \mathrm{D}^{\mathrm{b}}\left(\mathbb{P}^{2}, \mathcal{B}_{0}\right) .
$$

Note that, in view of [BMMS12, Proposition 2.9(i)], $\Xi_{3}\left(\mathbf{T}_{Y}\right)$ is the right orthogonal of the category generated by $\mathcal{B}_{1}$ in $\mathrm{D}^{\mathrm{b}}\left(\mathbb{P}^{2}, \mathcal{B}_{0}\right)$.

Remark 2.1. For all $m \in \mathbb{Z}$, we have $\Psi\left(\mathcal{O}_{\widetilde{Y}}(m h)\right)=0$ (see, for example, [BMMS12, Example 2.4]). Thus, if $F \in \mathbf{T}_{Y}$, then $\Xi_{3}(F)=\Psi(F)$.

\section{$2.2 \mathcal{B}_{0}$-modules and stability}

Our first goal is to study moduli spaces of stable $\mathcal{B}_{0}$-modules. In this section we present how the usual notion of stability extends to our more general situation.

Let $K\left(\mathbb{P}^{2}, \mathcal{B}_{0}\right):=K\left(\mathrm{D}^{\mathrm{b}}\left(\mathbb{P}^{2}, \mathcal{B}_{0}\right)\right)$ denote the Grothendieck group. For objects in $\mathrm{D}^{\mathrm{b}}\left(\mathbb{P}^{2}, \mathcal{B}_{0}\right)$ we can consider the Euler characteristic

$$
\chi(-,-):=\sum_{i}(-1)^{i} \operatorname{hom}_{\mathrm{D}^{\mathrm{b}\left(\mathbb{P}^{2}, \mathcal{B}_{0}\right)}}^{i}(-,-) .
$$

A class $[A] \in K\left(\mathbb{P}^{2}, \mathcal{B}_{0}\right)$ in the Grothendieck group is numerically trivial if $\chi([M],[A])=0$ for all $[M] \in K\left(\mathbb{P}^{2}, \mathcal{B}_{0}\right)$. We define the numerical Grothendieck group $\mathcal{N}\left(\mathbb{P}^{2}, \mathcal{B}_{0}\right)$ as the quotient of $K\left(\mathbb{P}^{2}, \mathcal{B}_{0}\right)$ by numerically trivial classes.

Given $K \in \mathrm{D}^{\mathrm{b}}\left(\mathbb{P}^{2}, \mathcal{B}_{0}\right)$, we define its Chern character as

$$
\operatorname{ch}(K):=\operatorname{ch}(\operatorname{Forg}(K)) \in K\left(\mathbb{P}^{2}\right) \otimes \mathbb{Q}=H^{*}\left(\mathbb{P}^{2}, \mathbb{Q}\right) \cong \mathbb{Q}^{\oplus 3},
$$

where Forg : $\mathrm{D}^{\mathrm{b}}\left(\mathbb{P}^{2}, \mathcal{B}_{0}\right) \rightarrow \mathrm{D}^{\mathrm{b}}\left(\mathbb{P}^{2}\right)$ is the functor forgetting the $\mathcal{B}_{0}$-action. By linearity the Chern character extends to $K\left(\mathbb{P}^{2}, \mathcal{B}_{0}\right)$; it factors through $\mathcal{N}\left(\mathbb{P}^{2}, \mathcal{B}_{0}\right)$.

Remark 2.2. (i) By [BMMS12, Proposition 2.12] we have $\mathcal{N}\left(\mathbb{P}^{2}, \mathcal{B}_{0}\right)=\mathbb{Z}\left[\mathcal{B}_{1}\right] \oplus \mathbb{Z}\left[\mathcal{B}_{0}\right] \oplus \mathbb{Z}\left[\mathcal{B}_{-1}\right]$. The Chern characters $\operatorname{ch}\left(\mathcal{B}_{-1}\right)=\left(4,-7, \frac{15}{2}\right), \operatorname{ch}\left(\mathcal{B}_{0}\right)=\left(4,-5, \frac{9}{2}\right)$, and $\operatorname{ch}\left(\mathcal{B}_{1}\right)=\left(4,-3, \frac{5}{2}\right)$ are linearly independent. Hence, the Chern character induces a group homomorphism $\mathcal{N}\left(\mathbb{P}^{2}, \mathcal{B}_{0}\right) \rightarrow K\left(\mathbb{P}^{2}\right)$ that is an isomorphism over $\mathbb{Q}$.

(ii) If $l \subseteq Y$ is a line and $\mathcal{I}_{l}$ is its ideal sheaf, by [BMMS12, Example 2.11], we have

$$
\begin{aligned}
{\left[\Xi_{3}\left(\mathcal{I}_{l}\right)\right] } & =\left[\mathcal{B}_{1}\right]-\left[\mathcal{B}_{0}\right] \in \mathcal{N}\left(\mathbb{P}^{2}, \mathcal{B}_{0}\right) \\
\operatorname{ch}\left(\Xi_{3}\left(\mathcal{I}_{l}\right)\right) & =(0,2,-2) .
\end{aligned}
$$

(iii) Note that $\left[\mathcal{B}_{2}\right]=\left[\mathcal{B}_{-1}\right]-3\left[\mathcal{B}_{0}\right]+3\left[\mathcal{B}_{1}\right]$ and $\left[\mathcal{B}_{-2}\right]=3\left[\mathcal{B}_{-1}\right]-3\left[\mathcal{B}_{0}\right]+\left[\mathcal{B}_{1}\right]$.

(iv) Given $[F]=x\left[\mathcal{B}_{-1}\right]+y\left[\mathcal{B}_{0}\right]+z\left[\mathcal{B}_{1}\right]$ or $\operatorname{ch}([F])=\left(r, c_{1}, \mathrm{ch}_{2}\right)$, we can compute the Euler characteristic as a $\mathcal{B}_{0}$-module with the following formulas:

$$
\begin{aligned}
\chi(F, F) & =x^{2}+y^{2}+z^{2}+3 x y+3 y z+6 x z \\
& =-\frac{7}{64} r^{2}-\frac{1}{4} c_{1}^{2}+\frac{1}{2} r \mathrm{ch}_{2} .
\end{aligned}
$$

(v) Let $F \in K\left(\mathbb{P}^{2}, \mathcal{B}_{0}\right)$ be such that $\operatorname{ch}(F)=(0,2 d, z)$. Then

$$
\chi\left(\mathcal{B}_{1}, F\right)=z+2 d \quad \text { and } \quad \chi\left(\mathcal{B}_{0}, F\right)=z+3 d .
$$

(vi) The Serre functor in $\mathrm{D}^{\mathrm{b}}\left(\mathbb{P}^{2}, \mathcal{B}_{0}\right)$ is given by $-\otimes_{\mathcal{B}_{0}} \mathcal{B}_{-1}[2]$ (see, for example, [BMMS12, Proposition 2.9]). 


\section{Martí Lahoz, Emanuele Macrì and PaOlo Stellari}

We define the Hilbert polynomial of a $\mathcal{B}_{0}$-module $G$ as the Hilbert polynomial of $\operatorname{Forg}(G)$ with respect to $\mathcal{O}_{\mathbb{P}^{2}}(h)$. Then, the notion of Gieseker (semi)stability is defined in the usual way. Moduli spaces of semistable $\mathcal{B}_{0}$-modules have been constructed by Simpson in [Sim94, Theorem 4.7].

We can also consider the slope stability for torsion-free sheaves in $\mathbf{C o h}\left(\mathbb{P}^{2}, \mathcal{B}_{0}\right)$. Indeed, we have two natural functions rank and degree on $\mathcal{N}\left(\mathbb{P}^{2}, \mathcal{B}_{0}\right)$ :

$$
\begin{aligned}
\operatorname{rk}: \mathcal{N}\left(\mathbb{P}^{2}, \mathcal{B}_{0}\right) \rightarrow \mathbb{Z}, & \operatorname{rk}(K):=\operatorname{rk}(\operatorname{Forg}(K)), \\
\operatorname{deg}: \mathcal{N}\left(\mathbb{P}^{2}, \mathcal{B}_{0}\right) \rightarrow \mathbb{Z}, & \operatorname{deg}(K):=\mathrm{c}_{1}(\operatorname{Forg}(K)) \cdot \mathrm{c}_{1}\left(\mathcal{O}_{\mathbb{P}^{2}}(h)\right) .
\end{aligned}
$$

Given $K \in \operatorname{Coh}\left(\mathbb{P}^{2}, \mathcal{B}_{0}\right)$ with $\operatorname{rk}(K) \neq 0$, we can define the slope $\mu(K):=\operatorname{deg}(K) / \operatorname{rk}(K)$ and the notion of $\mu$-(semi)stability in the usual way. When we say that $K$ is either torsion-free or torsion of dimension $d$, we always mean that $\operatorname{Forg}(K)$ has this property.

Remark 2.3. As the rank of $\mathcal{B}_{0}$ and that of $\mathcal{B}_{1}$ are both four, a consequence of [BMMS12, Lemma $2.13(\mathrm{i})]$ is that these two objects are $\mu$-stable. Moreover, all morphisms $\mathcal{B}_{0} \rightarrow \mathcal{B}_{1}$ are injective.

Lemma 2.4. Let $A, B \in \mathbf{C o h}\left(\mathbb{P}^{2}, \mathcal{B}_{0}\right)$ be such that $\operatorname{ch}(A)=\operatorname{ch}(B)$. Assume that one of the following two conditions is satisfied:

- either $A$ and $B$ are torsion-free sheaves and $\mu$-semistable, or

- $A$ and $B$ are torsion sheaves pure of dimension one and semistable.

Then $\operatorname{Ext}^{2}(A, B)=0$. If $A=B$ is actually stable, then $\chi(A, A) \leqslant 1$.

Proof. The first claim follows directly from Serre duality. Indeed, by Remark 2.2(vi), we have

$$
\operatorname{Ext}^{2}(A, B)=\operatorname{Hom}\left(B, A \otimes_{\mathcal{B}_{0}} \mathcal{B}_{-1}\right)^{\vee}=0,
$$

since $-\otimes_{\mathcal{B}_{0}} \mathcal{B}_{-1}$ preserves stability. For the second, simply observe that

$$
\chi(A, A)=\operatorname{hom}(A, A)-\operatorname{ext}^{1}(A, A)=1-\operatorname{ext}^{1}(A, A) \leqslant 1 .
$$

Bridgeland stability. We will need to study stability for objects in $\mathrm{D}^{\mathrm{b}}\left(\mathbb{P}^{2}, \mathcal{B}_{0}\right)$ which are not necessarily sheaves. To this end, we briefly recall the concept of Bridgeland stability condition. For all details we refer to [Bri07, KS08].

Definition 2.5. A (numerical, full) Bridgeland stability condition on $\mathrm{D}^{\mathrm{b}}\left(\mathbb{P}^{2}, \mathcal{B}_{0}\right)$ consists of a pair $\sigma=(Z, \mathbf{A})$, where

$-Z: \mathcal{N}\left(\mathbb{P}^{2}, \mathcal{B}_{0}\right) \rightarrow \mathbb{C}$

- A is the heart of a bounded $t$-structure on $\mathrm{D}^{\mathrm{b}}\left(\mathbb{P}^{2}, \mathcal{B}_{0}\right)$,

satisfying the following compatibilities:

(a) For all $0 \neq G \in \mathbf{A}$,

$$
Z(G) \in\left\{z \in \mathbb{C}^{*}: z=|z| \exp (i \pi \phi), 0<\phi \leqslant 1\right\} .
$$

(b) Harder-Narasimhan filtrations exist with respect to $\sigma$-stability, namely for any $0 \neq G \in \mathbf{A}$, there is a filtration in $\mathbf{A}$

$$
0=G_{0} \subset G_{1} \subset \ldots \subset G_{N}=G
$$

such that $F_{i}:=G_{i} / G_{i-1}$ is $\sigma$-semistable and $\phi\left(F_{1}\right)>\ldots>\phi\left(F_{N}\right)$.

(c) The support property holds, namely there exists a constant $C>0$ such that, for all $\sigma$ semistable $F \in \mathbf{A}$,

$$
\|F\| \leqslant C \cdot|Z(F)| .
$$




\section{ACM BUNDLES ON CUBIC THREEFOLDS}

The notion of $\sigma$-semistability in part (b) of Definition 2.5 can be made precise as follows. By part (a), any $0 \neq G \in \mathbf{A}$ has a phase $\phi(G):=\frac{1}{\pi} \arg (Z(G)) \in(0,1]$. The notion of $\sigma$-stability in part (b) is then given with respect to the phase: $G \in \mathbf{A}$ is $\sigma$-(semi) stable if, for all subobjects $G^{\prime} \subset G$ in $\mathbf{A}, \phi\left(G^{\prime}\right)<\phi(G)$ (respectively, $\phi\left(G^{\prime}\right) \leqslant \phi(G)$ ). Finally, we denoted by $\|-\|$ any norm in $\mathcal{N}\left(\mathbb{P}^{2}, \mathcal{B}_{0}\right) \otimes \mathbb{R}$. The support property is necessary for the deformation of stability conditions and for the existence of a well-behaved wall and chamber structure (this is [Bri08, Section 9]; the general statement we need is [BM11, Proposition 3.3]).

We will only need a special family of stability conditions on $\mathrm{D}^{\mathrm{b}}\left(\mathbb{P}^{2}, \mathcal{B}_{0}\right)$.

Definition 2.6. For $m \in \mathbb{R}_{>0}$, we define

$$
\begin{aligned}
Z_{m}: \mathcal{N}\left(\mathbb{P}^{2}, \mathcal{B}_{0}\right) & \longrightarrow \mathbb{C} \\
{[F] } & \longmapsto r m^{2}-\frac{9 r}{64}-\frac{c_{1}}{2}-\frac{\mathrm{ch}_{2}}{2}+m \sqrt{-1}\left(r+c_{1}\right),
\end{aligned}
$$

where $\operatorname{ch}([F])=\left(r, c_{1}, \operatorname{ch}_{2}\right)$.

By the explicit computations in Remark 2.2,

$$
\begin{aligned}
& Z_{m}\left(\left[\mathcal{B}_{0}\right]\right)=4 m^{2}-\frac{5}{16}-m \sqrt{-1}, \\
& Z_{m}\left(\left[\mathcal{B}_{1}\right]\right)=4 m^{2}-\frac{5}{16}+m \sqrt{-1}, \\
& Z_{m}\left(\left[\Xi_{3}\left(\mathcal{I}_{l}\right)\right]\right)=2 m \sqrt{-1}
\end{aligned}
$$

To define an abelian category which is the heart of a bounded $t$-structure on $\mathrm{D}^{\mathrm{b}}\left(\mathbb{P}^{2}, \mathcal{B}_{0}\right)$, let $\mathbf{T}, \mathbf{F} \subseteq \mathbf{C o h}(S, \beta)$ be the following two full additive subcategories: The non-trivial objects in $\mathbf{T}$ are the sheaves $A \in \mathbf{C o h}\left(\mathbb{P}^{2}, \mathcal{B}_{0}\right)$ such that their torsion-free part has Harder-Narasimhan factors (with respect to $\mu$-stability) of slope $\mu>-1$. A non-trivial twisted sheaf $A \in \mathbf{C o h}\left(\mathbb{P}^{2}, \mathcal{B}_{0}\right)$ is an object in $\mathbf{F}$ if $A$ is torsion-free and every $\mu$-semistable Harder-Narasimhan factor of $A$ has slope $\mu \leqslant-1$. It is easy to see that $(\mathbf{T}, \mathbf{F})$ is a torsion theory and following [Bri08], we define the heart of the induced $t$-structure as the abelian category

$$
\mathbf{A}:=\left\{A \in \mathrm{D}^{\mathrm{b}}\left(\mathbb{P}^{2}, \mathcal{B}_{0}\right): \mathcal{H}^{i}(A)=0 \text { for } i \notin\{-1,0\}, H^{-1}(A) \in \mathbf{F}, H^{0}(A) \in \mathbf{T}\right\} .
$$

By Remarks 2.2 and $2.3, \mathcal{B}_{0}[1], \mathcal{B}_{1}, \Xi_{3}\left(\mathcal{I}_{l}\right) \in \mathbf{A}$.

Lemma 2.7. The pair $\sigma_{m}:=\left(Z_{m}, \mathbf{A}\right)$ defines a stability condition in $\mathrm{D}^{\mathrm{b}}\left(\mathbb{P}^{2}, \mathcal{B}_{0}\right)$ for all $m>\frac{1}{4}$.

Proof. This follows exactly in the same way as in [Bri08, Proposition 7.1 and Section 11] and [Tod13, Proposition 3.13]. The only non-standard fact that we need is a Bogomolov-Gieseker inequality for torsion-free $\mu$-stable sheaves. This is precisely Lemma 2.4: for $A \in \mathbf{C o h}\left(\mathbb{P}^{2}, \mathcal{B}_{0}\right)$ torsion-free and $\mu$-stable, $\chi(A, A) \leqslant 1$ gives us the desired inequality.

By proceeding as in [Tod13, Section 3], to prove the lemma we only have to show property (a) in the definition of the stability condition. Let $A$ be a torsion-free $\mu$-stable sheaf. Assume further $\mu(A)=-1$, and so $\operatorname{Im}\left(Z_{m}([A])\right)=0$. By (2.2.2) and the fact that $r>0$, we have

$$
\operatorname{Re}\left(Z_{m}([A])\right)=r m^{2}-\frac{9 r}{64}-\frac{c_{1}}{2}-\frac{\mathrm{ch}_{2}}{2}=\frac{1}{r}\left(-\chi(A, A)+m^{2} r^{2}-\frac{1}{4}\left(r+c_{1}\right)^{2}\right) .
$$

We need to prove the inequality $\operatorname{Re}\left(Z_{m}([A])\right)>0$, namely $-\chi(A, A)+m^{2} r^{2}>0$. By $[\mathrm{BMMS} 12$, Lemma 2.13], $r \geqslant 4$, and so for all $m>\frac{1}{4}$, we have $\operatorname{Re}\left(Z_{m}([A])\right)>0$, as we wanted. 


\section{Martí Lahoz, Emanuele Macrì and PaOlo Stellari}

We also observe that all the arguments in [Tod08] generalize to the non-commutative setting (see also [Lie07, Lie06]). In particular, for all $m>\frac{1}{4}$, it makes sense to speak about moduli spaces of $\sigma_{m}$-semistable objects in $\mathbf{A}$ as Artin stacks (of finite-type over $\mathbb{C}$, if we fix the numerical class), and about moduli space of $\sigma_{m}$-stable objects as algebraic spaces.

Remark 2.8. As in [MS12, Lemma 5.5], the objects $\mathcal{B}_{0}[1]$ and $\mathcal{B}_{1}$ are $\sigma_{m}$-stable for all $m>\frac{1}{4}$.

\subsection{Moduli spaces of stable $\mathcal{B}_{0}$-modules: general results}

Keeping in mind (2.2.1), we are interested in the following moduli spaces of Gieseker semistable sheaves in $\left(\mathbb{P}^{2}, \mathcal{B}_{0}\right)$.

Definition 2.9. Let $d \geqslant 1$. We denote by $\mathfrak{M}_{d}$ the moduli space of semistable $\mathcal{B}_{0}$-modules with numerical class $d\left[\mathcal{B}_{1}\right]-d\left[\mathcal{B}_{0}\right]$, or equivalently, with Chern character $(0,2 d,-2 d)$. We denote by $\mathfrak{M}_{d}^{s} \subseteq \mathfrak{M}_{d}$ the open subset of stable $\mathcal{B}_{0}$-modules.

EXAmple 2.10. Let $C^{\prime} \subset Y$ be a rational curve of degree $d$. Note that, by using for example Theorem 62 of J. Starr's PhD thesis at Harvard University (2000), one can see that there exists a $2 d$-dimensional family of smooth rational curves of degree $d$ on $Y$. We can consider the following construction due to Kuznetsov [Kuz04, Lemma 4.6]. Set

$$
F_{d}:=\mathbf{L}_{\mathcal{O}_{Y}}\left(i_{*} \mathcal{O}_{C^{\prime}}(d-1)\right)[-1]=\operatorname{ker}\left(H^{0}\left(Y, i_{*} \mathcal{O}_{C^{\prime}}(d-1)\right) \otimes \mathcal{O}_{Y} \stackrel{\text { ev }}{\rightarrow} i_{*} \mathcal{O}_{C^{\prime}}(d-1)\right) \in \mathbf{T}_{Y},
$$

where $i: C^{\prime} \hookrightarrow Y$. Then $\Xi_{3}\left(F_{d}\right) \in\left\langle\mathcal{B}_{1}\right\rangle^{\perp}$. Suppose $C^{\prime} \cap l_{0}=\emptyset$. Denote by $j$ the composition $C^{\prime} \hookrightarrow \widetilde{Y} \stackrel{\pi}{\rightarrow} \mathbb{P}^{2}$ and suppose that, if we let $C:=j\left(C^{\prime}\right)$, the morphism $\left.j\right|_{C^{\prime}}$ is birational. As $C^{\prime}$ and $l_{0}$ do not intersect, we can argue exactly as in [BMMS12, Example 2.4]. In particular, using that $\Psi\left(\mathcal{O}_{\widetilde{Y}}(m h)\right)=0$ for all integers $m$, we conclude that

$$
\Xi_{3}\left(F_{d}\right) \cong j_{*}\left(\left.\mathcal{E}\right|_{C^{\prime}} \otimes \mathcal{O}_{C^{\prime}}(-1)\right) \otimes \mathcal{O}_{\mathbb{P}^{2}}(2 h) .
$$

So $\operatorname{Forg}\left(\Xi_{3}\left(F_{d}\right)\right)$ is a rank two torsion-free sheaf supported on $C$ and $\Xi_{3}\left(F_{d}\right) \in \mathfrak{M}_{d}^{s}$. The $d=1$ case is treated in Example 2.11 below. We will also use this example for $d=2$ and $d=3$. In such cases, there always exists a curve $C^{\prime} \subset Y$ with the properties above.

EXAMPLE 2.11. We can specialize the previous example to the case when $C^{\prime} \subset Y$ is a line $l$ which does not intersect $l_{0}$, namely $d=1$. In such a case, we have $F_{d} \cong I_{l}$ and

$$
\Xi_{3}\left(\mathcal{I}_{l}\right) \cong j_{*}\left(\left.\mathcal{E}\right|_{l}\right) \otimes \mathcal{O}_{\mathbb{P}^{2}}(h) \text {. }
$$

Moreover, we have an isomorphism as $\mathcal{O}_{\mathbb{P}^{2} \text {-modules }}$

$$
\Xi_{3}\left(\mathcal{I}_{l}\right) \cong \mathcal{O}_{l} \oplus \mathcal{O}_{l}(-h) \text {. }
$$

Indeed, by (2.2.1), the Chern character of $\Xi_{3}\left(\mathcal{I}_{l}\right)$ as an $\mathcal{O}_{\mathbb{P}^{2} \text {-module is }}$

$$
\operatorname{ch}\left(\Xi_{3}\left(\mathcal{I}_{l}\right)\right)=(0,2,-2) .
$$

Therefore, $\Xi_{3}\left(\mathcal{I}_{l}\right) \cong \mathcal{O}_{l}(a) \oplus \mathcal{O}_{l}(-1-a)$ for $a \in \mathbb{Z}_{\geqslant 0}$. Since, by [BMMS12, Lemma 4.8], we have

$$
0=\operatorname{Hom}_{\mathrm{D}^{\mathrm{b}}\left(\mathbb{P}^{2}, \mathcal{B}_{0}\right)}\left(\mathcal{B}_{0}(h), \Xi_{3}\left(\mathcal{I}_{l}\right)\right)=\operatorname{Hom}_{\mathbb{P}^{2}}\left(\mathcal{O}_{\mathbb{P}^{2}}, \mathcal{O}_{l}(a-1) \oplus \mathcal{O}_{l}(-2-a)\right),
$$

we deduce that $a=0$, as we wanted.

It is a standard fact (it follows, for example, as in [BM14, Example 9.5]) that the assignment

$$
\Upsilon: \mathfrak{M}_{d} \rightarrow\left|\mathcal{O}_{\mathbb{P}^{2}}(d)\right| \quad G \mapsto \operatorname{supp} \operatorname{Forg}(G) .
$$




\section{ACM BUNDLES ON CUBIC THREEFOLDS}

extends to a morphism which is well defined everywhere. Theorem A then becomes the following statement.

Theorem 2.12. The moduli space $\mathfrak{M}_{d}$ is irreducible, and for a general smooth curve $C \in\left|\mathcal{O}_{\mathbb{P}^{2}}(d)\right|$, we have

$$
\Upsilon^{-1}(C) \cong \bigsqcup_{2^{5 d-1}} J C
$$

where $J C=\left\{L \in \operatorname{Pic}(C) \mid L\right.$ is algebraically equivalent to $\left.\mathcal{O}_{C}\right\}$ is the Jacobian of $C$. Moreover, the stable locus $\mathfrak{M}_{d}^{s}$ is smooth of dimension $d^{2}+1$.

Before proceeding with the general proof which is carried out in the next section, we examine the easy case $d=1$.

Proposition 2.13. The moduli space $\mathfrak{M}_{1}=\mathfrak{M}_{1}^{s}$ is isomorphic to the Fano surface of lines $F(Y)$ blown up at the line $l_{0}$. In particular, $\mathfrak{M}_{1}$ is smooth and irreducible.

To prove Proposition 2.13, we use wall-crossing techniques from [BM14] for the family of Bridgeland stability conditions $\sigma_{m}$ of Lemma 2.7. The precise result we need is the following lemma, whose proof is exactly the same as that of [MS12, Lemma 5.7].

Lemma 2.14. Let $F \in \mathfrak{M}_{1}$.

(i) If $F \in\left\langle\mathcal{B}_{1}\right\rangle^{\perp}$, then $F$ is $\sigma_{m}$-stable for all $m>\frac{1}{4}$. Moreover, in this case $F \in \mathbf{A} \cap \Xi_{3}\left(\mathbf{T}_{Y}\right)$. By [BMMS12, Theorem 4.1], $F \cong \Xi_{3}\left(\mathcal{I}_{l}\right)$ for some line $l \neq l_{0}$ in $Y$.

(ii) If $F \notin\left\langle\mathcal{B}_{1}\right\rangle^{\perp}$, then $F$ sits in a short exact sequence

$$
0 \rightarrow \mathcal{B}_{0} \rightarrow \mathcal{B}_{1} \rightarrow F \rightarrow 0
$$

and $F$ becomes $\sigma_{m}$-semistable for $m=\frac{\sqrt{5}}{8}$ with Jordan-Hölder filtration

$$
\mathcal{B}_{1} \rightarrow F \rightarrow \mathcal{B}_{0}[1] \text {. }
$$

By [BMMS12, Example 2.11], the object $\Xi_{3}\left(\mathcal{I}_{l_{0}}\right)$ sits in the distinguished triangle

$$
\mathcal{B}_{0}[1] \rightarrow \Xi_{3}\left(\mathcal{I}_{l_{0}}\right) \rightarrow \mathcal{B}_{1}
$$

which is the Harder-Narasimhan filtration of $\Xi_{3}\left(\mathcal{I}_{l_{0}}\right)$ for $m>m_{0}:=\frac{\sqrt{5}}{8}$. Thus, all such extensions (2.3.3) get contracted to $\Xi_{3}\left(\mathcal{I}_{l_{0}}\right)$, which is indeed $\sigma_{m}$-stable for $m \in\left(m_{0}-\varepsilon, m_{0}\right)$. The wall-crossing phenomenon described in [MS12, Section 5.2] carries over and this proves Proposition 2.13 .

\subsection{Proof of Theorem 2.12}

The argument is divided into various steps.

Step 1: Deformation theory. For any $G \in \mathfrak{M}_{d}$, we have $\chi(G, G)=-d^{2}$. Hence, to prove that $\mathfrak{M}_{d}^{s}$ is smooth of dimension $d^{2}+1$, it is enough to show that it is non-empty and that

$$
\operatorname{Hom}_{\mathrm{D}^{\mathrm{b}}\left(\mathbb{P}^{2}, \mathcal{B}_{0}\right)}^{2}(G, G)=0
$$

for any $G \in \mathfrak{M}_{d}$. The fact that $\mathfrak{M}_{d}^{s}$ is non-empty is a consequence of the next step. The vanishing of $\operatorname{Hom}_{\mathrm{D}^{\mathrm{b}}\left(\mathbb{P}^{2}, \mathcal{B}_{0}\right)}^{2}(G, G)$ follows directly from Lemma 2.4.

Step 2: Fibers of $\Upsilon: \mathfrak{M}_{d} \rightarrow\left|\mathcal{O}_{\mathbb{P}^{2}}(d)\right|$. We claim that for a smooth curve $C \in\left|\mathcal{O}_{\mathbb{P}^{2}}(d)\right|$, we have

$$
\Upsilon^{-1}(C) \cong \bigsqcup_{2^{5 d-1}} \operatorname{Pic}^{0}(C)
$$




\section{Martí Lahoz, Emanuele Macrì and PaOlo Stellari}

Recall that the conic fibration $\pi$ degenerates along a smooth quintic $\Delta \subset \mathbb{P}^{2}$. We denote $\left.\Delta\right|_{C}$ by $\sum_{i=1}^{5 d} p_{i}$ (the points are possibly non-distinct) and, abusing notation, we set $\frac{1}{2} p_{i}$ to be the section in $\widehat{C}$ corresponding to the second root of $p_{i}$. As in Proposition 1.14, we can consider the stack $\widehat{\mathbb{P}^{2}}$ over $\mathbb{P}^{2}$ of second roots of $\mathcal{O}_{\mathbb{P}^{2}}(\Delta)$ along the section $\Delta$. We denote by $\psi: \widehat{\mathbb{P}^{2}} \rightarrow \mathbb{P}^{2}$ the natural projection. We then have an equivalence of abelian categories

$$
\psi_{*}: \operatorname{Coh}\left(\widehat{\mathbb{P}^{2}}, \mathcal{A}_{0}\right) \rightarrow \operatorname{Coh}\left(\mathbb{P}^{2}, \mathcal{B}_{0}\right) .
$$

Given a smooth curve $C \subset \mathbb{P}^{2}$ we can restrict this construction to $\psi: \widehat{C} \rightarrow C$, where $\widehat{C}$ is a twisted curve (stack of second roots of $\left(C,\left.\Delta\right|_{C}\right)$ ). The restriction $\left.\mathcal{A}_{0}\right|_{\widehat{C}}$ is a sheaf of (trivial) Azumaya algebras; that is, there exists a vector bundle of rank two, $E_{C, 0} \in \mathbf{C o h}(\widehat{C})$, such that $\left.\mathcal{A}_{0}\right|_{\widehat{C}}=\mathcal{E} n d\left(E_{C, 0}\right)$ (see, for example, [Kuz08, Corollary 3.16]) and

$$
\begin{aligned}
& \operatorname{Coh}(\widehat{C}) \stackrel{\sim}{\longrightarrow} \operatorname{Coh}\left(\widehat{C},\left.\mathcal{A}_{0}\right|_{\widehat{C}}\right) \stackrel{\sim}{\longrightarrow} \operatorname{Coh}\left(C,\left.\mathcal{B}_{0}\right|_{C}\right) \\
& G \longmapsto G \otimes E_{C, 0}^{\vee} \longmapsto \psi_{*}\left(G \otimes E_{C, 0}^{\vee}\right)
\end{aligned}
$$

is an equivalence of categories. In particular,

$$
\left.\mathcal{B}_{0}\right|_{C}=\psi_{*}\left(\mathcal{E} n d\left(E_{C, 0}\right)\right) .
$$

Moreover, there certainly exists an $M \in \operatorname{Pic}(\widehat{C})$ such that $\operatorname{ch}_{2}\left(\psi_{*}\left(E_{C, 0}^{\vee} \otimes M\right)\right)=-2 d$ as an

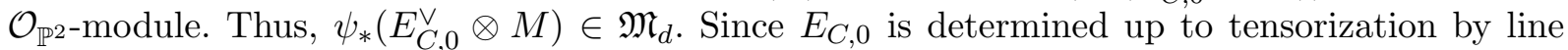
bundles, we can assume directly that $\psi_{*} E_{C, 0}^{\vee} \in \mathfrak{M}_{d}$.

As $E_{C, 0}$ is a rank two vector bundle on $\widehat{C}$, it is clear that the fiber of $\Upsilon$ over the smooth curve $C$ consists of line bundles on $\widehat{C}$. By [Cad07, Corollary 3.1.2], an invertible sheaf on $\widehat{C}$ is of the form $\psi^{*} L \otimes \mathcal{O}_{\widehat{C}}\left(\sum_{i=1}^{5 d} \frac{\lambda_{i}}{2} p_{i}\right)$, where $L \in \operatorname{Pic}(C)$ and $\lambda_{i} \in\{0,1\}$. On the other hand, as $E_{C, 0}^{\vee}$ has rank two, we have

$$
\operatorname{ch}_{2}\left(\psi_{*}\left(E_{C, 0}^{\vee} \otimes \psi^{*} L \otimes \mathcal{O}_{\widehat{C}}\left(\sum_{i=1}^{5 d} \frac{\lambda_{i}}{2} p_{i}\right)\right)\right)=\operatorname{ch}_{2}\left(\psi_{*}\left(E_{C, 0}^{\vee}\right)\right)
$$

as objects in $\mathrm{D}^{\mathrm{b}}\left(\mathbb{P}^{2}\right)$ with $L \in \operatorname{Pic}(C)$ and $\lambda_{i} \in\{0,1\}$, if and only if $2 \operatorname{deg} L+\sum_{i=1}^{5 d} \lambda_{i}=0$.

Let $J$ be the set of all subsets of $\{1, \ldots, 5 d\}$ of even cardinality and, for $I \in J$, set $\tau_{I}$ to be the cardinality of $I$. Then the discussion above can be rewritten as

$$
\Upsilon^{-1}(C)=\bigsqcup_{I \in J}\left\{\psi_{*}\left(E_{C, 0}^{\vee} \otimes \psi^{*} L \otimes \mathcal{O}_{\widehat{C}}\left(\sum_{i \in I} \frac{1}{2} p_{i}\right)\right): L \in \operatorname{Pic}^{-\tau_{I}}(C)\right\} .
$$

Hence

$$
\Upsilon^{-1}(C) \cong \bigsqcup_{I \in J} \operatorname{Pic}^{-\tau_{I}}(C)
$$

which is precisely (2.4.1), because $J$ has cardinality $2^{5 d-1}$.

Step 3: $\mathfrak{M}_{d}$ is irreducible. To prove the irreducibility of $\mathfrak{M}_{d}$, we follow the same strategy as in [KLS06]. We first prove that $\mathfrak{M}_{d}$ is connected by simply following the same argument as in the proof of [KLS06, Theorem 4.4]. Indeed, by Proposition 2.13, we know that $\mathfrak{M}_{1}$ is connected. Now if $1 \leqslant d_{1} \leqslant d_{2}<d$ and $d_{1}+d_{2}=d$, we have the natural maps $\varphi_{d_{1}, d_{2}}: \mathfrak{M}_{d_{1}} \times \mathfrak{M}_{d_{2}} \rightarrow \mathfrak{M}_{d}$ sending the pair $\left(E_{1}, E_{2}\right)$ to $E_{1} \oplus E_{2}$. Their images coincide with the semistable locus of $\mathfrak{M}_{d}$, which is then connected by induction. The existence of a connected component in $\mathfrak{M}_{d}$ consisting of 


\section{ACM BUNDLES ON CUBIC THREEFOLDS}

purely stable objects can be excluded by using an argument of Mukai as in the proof of [KLS06, Theorem 4.1]. For the convenience of the reader, let us outline here some details.

The aim is to show that, if there exists a connected component $X$ of the moduli space $\mathfrak{M}_{d}$ consisting only of stable sheaves, then $X \cong \mathfrak{M}_{d}$. Take a point $F \in X$ and assume that there is a $G \in \mathfrak{M}_{d} \backslash X$. The arguments in [KLS06, Lemma 4.2] and in [KLS06, $\S 4.3$ ] show that we can essentially assume that there is a universal family $\mathcal{F} \in \mathbf{C o h}\left(X \times \mathfrak{M}_{d}\right)$ with two projections $p: X \times \mathfrak{M}_{d} \rightarrow X$ and $q: X \times \mathfrak{M}_{d} \rightarrow \mathfrak{M}_{d}$. By Lemma 2.4, we have $\operatorname{Hom}_{\mathrm{D}^{\mathrm{b}}\left(\mathbb{P}^{2}, \mathcal{B}_{0}\right)}^{2}\left(G^{\prime}, F^{\prime}\right)=0$ for all $G^{\prime}, F^{\prime} \in \mathfrak{M}_{d}$. Hence a computation of local groups Ext shows that

$$
\operatorname{Ext}_{p}^{0}\left(q^{*} G, \mathcal{F}\right)=\operatorname{Ext}_{p}^{2}\left(q^{*} G, \mathcal{F}\right)=0,
$$

while $\operatorname{Ext}_{p}^{1}\left(q^{*} G, \mathcal{F}\right)$ is locally free of rank $d^{2}<1+d^{2}=\operatorname{dim}(X)$ (the last equality follows again from Step 1). If we replace $G$ by $F$, we get a complex of $\mathcal{O}_{X}$-modules

$$
0 \longrightarrow A_{0} \stackrel{\alpha}{\longrightarrow} A_{1} \stackrel{\beta}{\longrightarrow} A_{2} \longrightarrow 0
$$

such that $H^{i}\left(A^{\bullet}\right) \cong \operatorname{Ext}_{p}^{i}\left(q^{*} F, \mathcal{F}\right)$ (more generally, this holds for any base change $S \rightarrow X$ ).

It turns out that the point $F \in X$ is the degeneracy locus of the map $\alpha$ (see [KLS06, Lemma 4.3]). Thus, blowing up $X$ at $F$, we get $f: Z \rightarrow X$ providing, as in (2.4.2), a new complex of $\mathcal{O}_{Z}$-modules

$$
A_{0}^{\prime} \stackrel{\alpha^{\prime}}{\longrightarrow} A_{1}^{\prime} \stackrel{\beta^{\prime}}{\longrightarrow} A_{2}^{\prime}
$$

with an inclusion $f^{*} A_{0} \subseteq A_{0}^{\prime}$. Let $D$ be the exceptional divisor in $Z$ and let $W^{\prime}$ be the middle cohomology of

$$
0 \longrightarrow A_{0}^{\prime} \longrightarrow f^{*} A_{1} \longrightarrow A_{2}^{\prime} \longrightarrow 0
$$

If $M:=A_{0}^{\prime} / f^{*} A_{0}$, then, by the same computations of the Chern classes as in [KLS06, $\left.\S 4.4\right]$, we get $c\left(W^{\prime}\right)=f^{*} c(W) \cdot c(-M)$. As the ranks of $W$ and $W^{\prime}$ are smaller than $\operatorname{dim}(X)$, one gets a contradiction as $c_{\operatorname{dim}(X)}\left(W^{\prime}\right)=0$ while, using that $M \cong \mathcal{O}_{D}(D)$, one shows that the component in degree $\operatorname{dim}(X)$ of $f^{*} c(W) \cdot c(-M)$ is not trivial.

Hence, to conclude that $\mathfrak{M}_{d}$ is irreducible, it is enough to show that it is normal. Since, by Lemma 2.4, $\operatorname{Hom}_{\mathrm{D}^{\mathrm{b}}\left(\mathbb{P}^{2}, \mathcal{B}_{0}\right)}^{2}(G, G)=0$ for all $G \in \mathfrak{M}_{d}$, the Kuranishi map is trivial. Following then the notation of [KLS06, $\S 2.7$ ], the quadratic part $\mu$ of the Kuranishi map is also trivial and the null-fiber $F=\mu^{-1}(0)$ coincides with $\operatorname{Hom}_{\mathrm{D}^{\mathrm{b}}\left(\mathbb{P}^{2}, \mathcal{B}_{0}\right)}^{1}(G, G)$, which is obviously normal. Then we can apply [KLS06, Proposition 3.8]; that is, if we consider a slice $S$ of an orbit of a semistable point $[q]$ of the corresponding Quot-space, we have that $\mathcal{O}_{S,[q]}$ is a normal domain. Since being normal is an open property, we can use the arguments in the proof of [KLS06, Proposition 3.11] to prove that the locus $R^{s s}$ of semistable points of the Quot-space is normal. A GIT-quotient of a normal scheme is normal. Hence, $\mathfrak{M}_{d}$ is normal, since it is a GIT-quotient of $R^{s s}$.

This finally concludes the proof of Theorem 2.12 .

Remark 2.15. When $d=1$, the map $\Upsilon$ has a very natural and well-known geometrical interpretation. In fact, given a $\mathcal{B}_{0}$-module $F$ supported on a general line $l \subset \mathbb{P}^{2}$, we can consider all the lines $l^{\prime}$ in $Y$ such that $\Xi_{3}\left(\mathcal{I}_{l^{\prime}}\right) \cong F$. By Proposition 2.13, we have to count the number of lines $l^{\prime}$ that map to $l$ via the projection from $l_{0}$ (where the lines that intersect $l_{0}$ are mapped to the projection of the tangent space of the intersection point). The lines that intersect $l_{0}$ form an Abel-Prym curve in $F(Y)$, so they do not dominate $\left|\mathcal{O}_{\mathbb{P}^{2}}(1)\right|$. Hence, we need only to count the skew lines to $l_{0}$ that map to $l$. The preimage of $l$ via the projection is a cubic surface, so it 


\section{Martí Lahoz, Emanuele Macrì and PaOlo Stellari}

contains 27 lines. The line $l$ intersects the degeneration quintic $\Delta$ in five points, which give us five coplanar pairs of lines intersecting $l_{0}$. Hence we have $27-10-1=2^{4}$ lines skew to $l_{0}$ that project to $l$. Indeed, if $\mathrm{Bl}_{l_{0}} F(Y)$ is the blow-up of $F(Y)$ along $l_{0}$, we have a finite morphism $\mathrm{Bl}_{l_{0}} F(Y) \rightarrow\left|\mathcal{O}_{\mathbb{P}^{2}}(1)\right|$ which is $2^{4}: 1$ (see, for example, [Beau81, Proof of Theorem 4]).

For applications to stable sheaves on cubic threefolds, as in Lemma 2.14, we consider the subset

$$
\mathfrak{N}_{d}:=\left\langle\mathcal{B}_{1}\right\rangle^{\perp} \cap \mathfrak{M}_{d}
$$

Lemma 2.16. The subset $\mathfrak{N}_{d}$ is well defined; namely, it does not depend on the chosen representative in the $S$-equivalence class.

Proof. First of all, we observe that by Remark 2.2(v), for all $A \in \mathfrak{M}_{d}$, we have $\chi\left(\mathcal{B}_{1}, A\right)=0$. Moreover, by Serre duality, $\operatorname{Hom}^{2}\left(\mathcal{B}_{1}, A\right)=0$, since $A$ is torsion. Hence, we have $\operatorname{Hom}\left(\mathcal{B}_{1}, A\right)=0$ if and only if $A \in \mathfrak{N}_{d}$.

Let $A \in \mathfrak{M}_{d}$ and let $A_{1}, \ldots, A_{m}$ be its Jordan-Hölder factors. It is enough to show the following claim: $A \in \mathfrak{N}_{d}$ if and only if $A_{i} \in \mathfrak{N}_{d}$ for all $i$. As observed in Step 3 of the proof of Theorem 2.12, we have $A_{i} \in \mathfrak{M}_{d_{i}}$ for some $d_{i}>0$. The claim then directly follows from the long exact sequence in cohomology (by applying $\operatorname{Hom}\left(\mathcal{B}_{1},-\right)$ to the Jordan-Hölder filtration of $A$ ) and by the previous observation.

By semi-continuity, the condition of belonging to $\mathfrak{N}_{d}$ is open in families. We also have the following result.

Proposition 2.17. The subset $\mathfrak{N}_{d}$ is non-empty and dense in $\mathfrak{M}_{d}$.

Proof. This is a well-known general fact. The proof we give here mimics [BM13, Theorem 2.15]. We first recall that, as proved in Step 3 of the proof of Theorem 2.12, by considering the maps $\phi_{d_{1}, d_{2}}: \mathfrak{M}_{d_{1}} \times \mathfrak{M}_{d_{2}} \rightarrow \mathfrak{M}_{d}$, the subset $\mathfrak{M}_{d}^{s}$ consisting of stable sheaves in $\mathfrak{M}_{d}$ is open, non-empty, and dense.

We can now proceed by induction on $d$. The case $d=1$ is precisely Lemma 2.14.

Assume then $d>1$, and let $A_{d-1} \in \mathfrak{N}_{d-1}$. Since $\mathfrak{M}_{d}^{s}$ is non-empty, we can assume that $A_{d-1}$ is stable. Then we can find $A_{1} \in \mathfrak{N}_{1}$ such that $\operatorname{Hom}\left(A_{d-1}, A_{1}\right)=\operatorname{Hom}\left(A_{1}, A_{d-1}\right)=0$ (in fact, any $A_{1} \in \mathfrak{N}_{1}$ works, since $A_{d-1}$ is stable). By Remark 2.2(v), we have

$$
\chi\left(A_{1}, A_{d-1}\right)=(d-1) \chi\left(A_{1}, A_{1}\right)=-(d-1)<0 .
$$

Hence, $\operatorname{Ext}^{1}\left(A_{1}, A_{d-1}\right) \neq 0$. Consider a non-trivial extension

$$
0 \rightarrow A_{d-1} \rightarrow A_{d} \rightarrow A_{1} \rightarrow 0 .
$$

Then $A_{d} \in \mathfrak{N}_{d}$ and $\operatorname{Hom}\left(A_{d}, A_{d}\right) \cong \mathbb{C}$, namely $A_{d}$ is a simple sheaf. Since, by Lemma 2.4, $\operatorname{Ext}^{2}\left(A_{d}, A_{d}\right)=0$, we can consider a maximal-dimensional family of simple sheaves containing $A_{d}$. Hence, since both being stable and belonging to $\mathfrak{N}_{d}$ are open properties, we have that

$$
\emptyset \neq \mathfrak{N}_{d} \cap \mathfrak{M}_{d}^{s} \subset \mathfrak{M}_{d}^{s}
$$

is an open subset, and therefore dense. Since $\mathfrak{M}_{d}^{s}$ is dense in $\mathfrak{M}_{d}$, this concludes the proof.

\subsection{Ulrich bundles}

We now apply the results on $\mathcal{B}_{0}$-modules of the previous section to study Ulrich bundles on a cubic threefold $Y$. The goal is to prove Theorem B from the introduction. 


\section{ACM BUNDLES ON CUBIC THREEFOLDS}

Definition 2.18. An ACM bundle $F$ on $Y$ is called Ulrich if the graded module

$$
H_{*}^{0}(Y, F):=\bigoplus_{m \in \mathbb{Z}} H^{0}(Y, F(m H))
$$

has $3 \operatorname{rk}(F)$ generators in degree one.

We refer to [CHGS12, Section 1] for the basic properties of Ulrich bundles on projective varieties. In particular, we recall the following presentation of stable Ulrich bundles due to the Hartshorne-Serre construction.

Lemma 2.19. A stable Ulrich bundle $F$ of rank $r$ on a cubic threefold $Y$ admits the following presentation:

$$
0 \rightarrow \mathcal{O}_{Y}(-H)^{\oplus r-1} \rightarrow F \rightarrow \mathcal{I}_{C} \otimes \mathcal{O}_{Y}((r-1) H) \rightarrow 0
$$

where $C$ is a smooth connected curve of degree $\left(3 r^{2}-r\right) / 2$ and arithmetic genus $r^{3}-2 r^{2}+1$.

Proof. By definition, $F(H)$ is generated by global sections, so $G:=\operatorname{coker}\left(\mathcal{O}_{Y}(-H)^{\oplus r-1} \hookrightarrow F\right)$ is a torsion-free sheaf of rank one. By choosing the sections appropriately, we get $G=\mathcal{I}_{C} \otimes \mathcal{O}_{Y}(s H)$, where $C \subset Y$ is a smooth curve. By [CHGS12, Lemma 2.4(iii)], we have $c_{1}(F(H))=r$, so $s=r-1$. Since $h^{1}\left(Y, \mathcal{I}_{C}\right)=0$, we know that $C$ is connected. By [CH11, Proposition 3.7], we have $\operatorname{deg} C=\frac{3 r^{2}-r}{2}$ and by Riemann-Roch, we get $p_{a}(C)=r^{3}-2 r^{2}+1$.

From Lemma 2.19, it is standard to compute the Chern character of an Ulrich bundle $F$ of rank $r$ by using Hirzebruch-Riemann-Roch:

$$
\operatorname{ch}(F)=(r, 0,-r \cdot l, 0),
$$

where $l$ denotes the class of a line in $Y$.

Remark 2.20. Notice that, in their definition of Ulrich bundles [CHGS12, Definition 2.1], Casanellas and Hartshorne impose the generators to be in degree zero. Hence, their Ulrich bundles can be obtained from ours by twisting by $\mathcal{O}_{Y}(H)$ and vice versa. We prefer this normalization, since then Ulrich bundles are balanced ACM bundles (recall Definition 1.5). Moreover, with this normalization, instanton bundles of minimal charge (see Definition 3.7 and the subsequent comments) are also Ulrich bundles.

Denote by $\mathfrak{M}_{r}^{s U}$ the moduli space of stable Ulrich bundles of rank $r \geqslant 2$. It is smooth of dimension $r^{2}+1$ since for any such bundle $E$, we have $\operatorname{dim}_{\operatorname{Ext}^{1}}(E, E)=r^{2}+1$ while $\operatorname{dim} \operatorname{Ext}^{2}(E, E)=0$.

To prove that $\mathfrak{M}_{r}^{s U}$ is non-empty, the strategy is to show the existence of low rank Ulrich bundles $(r=2,3)$ and then use a "standard" deformation argument [CHGS12, Theorem 5.7]. The existence of rank two Ulrich bundles is well known [Dru00, MT01]. They usually appear in the literature as instanton bundles (see Section 3). In [CHGS12] the authors construct rank three Ulrich bundles, relying on the existence of an ACM curve on $Y$ of degree twelve and genus ten (see Lemma 2.19). The existence of such curves is proved, using Macaulay2, by Geiß and Schreyer in the appendix, only for a generic cubic threefold.

Our approach to construct Ulrich bundles of rank three is different (for completeness we also construct rank two Ulrich bundles). In particular, we do not use the Hartshorne-Serre construction (see Lemma 2.19), but the structure of conic fibration of a blow-up of $Y$. We have computed the image in $\mathrm{D}^{\mathrm{b}}\left(\mathbb{P}^{2}, \mathcal{B}_{0}\right)$ of the ideal sheaves of lines in $Y$ in Example 2.11. We can therefore consider extensions of them, and use deformation theory to cover the subset $\mathfrak{N}_{d} \subset \mathfrak{M}_{d}$ 


\section{Martí Lahoz, Emanuele Macrì and PaOlo Stellari}

(for $d=2,3$ ). If $G$ is a general sheaf in $\mathfrak{N}_{d}$, then the object $\Xi_{3}^{-1}(G)$ will be a stable ACM bundle of rank $d$, which will automatically be Ulrich.

The advantage of our approach is that by using the category $\mathbf{T}_{Y}$ we are able to reduce all computations to the category $\mathrm{D}^{\mathrm{b}}\left(\mathbb{P}^{2}, \mathcal{B}_{0}\right)$, via the functor $\Xi_{3}$. Thus, the existence result needed goes back to Theorem 2.12.

Given $G \in \mathfrak{N}_{d}$, we want to study $\Xi_{3}^{-1}(G) \in \mathbf{T}_{Y}$. In order to show that it is an ACM bundle we want to see how the vanishings in Lemma 1.9 can be checked in $\mathrm{D}^{\mathrm{b}}\left(\mathbb{P}^{2}, \mathcal{B}_{0}\right)$.

LEMma 2.21. We have the following natural isomorphisms:

$$
\begin{aligned}
\operatorname{RHom}_{\mathrm{D}^{\mathrm{b}}(Y)}\left(\mathcal{O}_{Y}(2 H), F\right)[2] & \cong \operatorname{RHom}_{\mathrm{D}^{\mathrm{b}}\left(\mathbb{P}^{2}, \mathcal{B}_{0}\right)}\left(\Omega_{\mathbb{P}^{2}}(2 h) \otimes \mathcal{B}_{0}, \Xi_{3}(F)\right) \\
& \cong \operatorname{RHom}_{\mathrm{D}^{\mathrm{b}}\left(\mathbb{P}^{2}\right)}\left(\Omega_{\mathbb{P}^{2}}(2 h), \Xi_{3}(F)\right) \\
\operatorname{RHom}_{\mathrm{D}^{\mathrm{b}}(Y)}\left(\mathcal{O}_{Y}(-H), F\right) & \cong \operatorname{RHom}_{\mathrm{D}^{\mathrm{b}}\left(\mathbb{P}^{2}, \mathcal{B}_{0}\right)}\left(\mathcal{B}_{-1}, \Xi_{3}(F)\right) \\
& \cong \operatorname{RHom}_{\mathrm{D}^{\mathrm{b}}\left(\mathbb{P}^{2}\right)}\left(\mathcal{O}_{\mathbb{P}^{2}}, \operatorname{Forg}\left(\Xi_{3}\left(F \otimes_{\mathcal{B}_{0}} \mathcal{B}_{1}\right)\right)\right)
\end{aligned}
$$

for all $F \in \mathbf{T}_{Y}$.

Proof. For the first series of isomorphisms, we start with the following chain of natural isomorphisms, which follows directly from the definitions:

$$
\begin{aligned}
& \operatorname{RHom}_{\mathrm{D}^{\mathrm{b}}(Y)}\left(\mathcal{O}_{Y}(2 H), F\right) \cong \operatorname{RHom}_{\mathrm{D}^{\mathrm{b}}(Y)}\left(\mathcal{O}_{Y}(2 H), \Xi_{3}^{-1}\left(\Xi_{3}(F)\right)\right) \\
& \cong \operatorname{RHom}_{\mathrm{D}^{\mathrm{b}}(Y)}\left(\mathcal{O}_{Y}(2 H), \sigma_{*} \circ \mathbf{R}_{\mathcal{O}_{\widetilde{Y}}(-h)} \circ \Phi \circ \Xi_{3}(F)\right) \\
& \cong \operatorname{RHom}_{\mathrm{D}^{\mathrm{b}}\left(\mathbb{P}^{2}, \mathcal{B}_{0}\right)}\left(\Psi \circ \mathbf{L}_{\mathcal{O}_{\widetilde{Y}}(H)}\left(\mathcal{O}_{\widetilde{Y}}(2 H)\right), \Xi_{3}(F)\right) \text {. }
\end{aligned}
$$

By (1.1.1), $\mathbf{L}_{\mathcal{O}_{\widetilde{Y}}(H)} \mathcal{O}_{\widetilde{Y}}(2 H)$ is given by

$$
\mathbf{L}_{\mathcal{O}_{\widetilde{Y}}(H)} \mathcal{O}_{\widetilde{Y}}(2 H)=\operatorname{cone}\left(\mathcal{O}_{\widetilde{Y}}(H)^{\oplus 5} \stackrel{\text { ev }}{\longrightarrow} \mathcal{O}_{\widetilde{Y}}(2 H)\right)
$$

By definition of $\Psi$ (1.3.2), we have the following two exact triangles in $\mathrm{D}^{\mathrm{b}}\left(\mathbb{P}^{2}, \mathcal{B}_{0}\right)$ :

$$
\begin{aligned}
\mathcal{B}_{1}[1] & \rightarrow \mathcal{B}_{0}(h) \otimes q_{*} \mathcal{O}_{\widetilde{\mathbb{P}} 4}(H)[1] \rightarrow \Psi\left(\mathcal{O}_{\widetilde{Y}}(2 H)\right), \\
\mathcal{B}_{1} \otimes q_{*} \mathcal{O}_{\widetilde{\mathbb{P}}}(-H)[1] \rightarrow \mathcal{B}_{0}(h)[1] & \rightarrow \Psi\left(\mathcal{O}_{\widetilde{Y}}(H)\right) .
\end{aligned}
$$

Since $\Xi_{3}(F) \in\left\langle\mathcal{B}_{1}\right\rangle^{\perp}$ and $q_{*} \mathcal{O}_{\widetilde{\mathbb{P}}^{4}}(H)=\mathcal{O}_{\mathbb{P}^{2}}^{\oplus 2} \oplus \mathcal{O}_{\mathbb{P}^{2}}(h)$, we have

$$
\begin{aligned}
\operatorname{RHom}_{\mathrm{D}^{\mathrm{b}}\left(\mathbb{P}^{2}, \mathcal{B}_{0}\right)}\left(\Psi\left(\mathcal{O}_{\widetilde{Y}}(2 H)\right), \Xi_{3}(F)\right) & =\operatorname{RHom}_{\mathrm{D}^{\mathrm{b}}\left(\mathbb{P}^{2}, \mathcal{B}_{0}\right)}\left(\mathcal{B}_{0}(h) \otimes q_{*} \mathcal{O}_{\widetilde{\mathbb{P}}^{4}}(H)[1], \Xi_{3}(F)\right) \\
& =\operatorname{RHom}_{\mathrm{D}^{\mathrm{b}}\left(\mathbb{P}^{2}, \mathcal{B}_{0}\right)}\left(\mathcal{B}_{0} \otimes\left(\mathcal{O}_{\mathbb{P}^{2}}(h)^{\oplus 2} \oplus \mathcal{O}_{\mathbb{P}^{2}}(2 h)\right)[1], \Xi_{3}(F)\right),
\end{aligned}
$$

and since $q_{*} \mathcal{O}_{\widetilde{\mathbb{P}}^{4}}(-H)=0$,

$$
\operatorname{RHom}_{\mathrm{D}^{\mathrm{b}}\left(\mathbb{P}^{2}, \mathcal{B}_{0}\right)}\left(\Psi \mathcal{O}_{\widetilde{Y}}(H), \Xi_{3} F\right)=\operatorname{RHom}_{\mathrm{D}^{\mathrm{b}}\left(\mathbb{P}^{2}, \mathcal{B}_{0}\right)}\left(\mathcal{B}_{0}(h)[1], \Xi_{3}(F)\right) .
$$

Therefore, combining (2.5.3) and (2.5.4) we have

$$
\begin{aligned}
& \operatorname{RHom}_{\mathrm{D}^{\mathrm{b}}(Y)}\left(\mathcal{O}_{Y}(2 H), F\right) \cong \\
& \cong \operatorname{RHom}_{\mathrm{D}^{\mathrm{b}}\left(\mathbb{P}^{2}, \mathcal{B}_{0}\right)}\left(\mathcal{B}_{0} \otimes \operatorname{cone}\left(\mathcal{O}_{\mathbb{P}^{2}}(h)^{\oplus 5} \stackrel{\mathrm{ev}}{\rightarrow} \mathcal{O}_{\mathbb{P}^{2}}(h)^{\oplus 2} \oplus \mathcal{O}_{\mathbb{P}^{2}}(2 h)\right)[1], \Xi_{3}(F)\right) \\
& \cong \mathrm{RHom}_{\mathrm{D}^{\mathrm{b}}\left(\mathbb{P}^{2}, \mathcal{B}_{0}\right)}\left(\mathcal{B}_{0} \otimes \Omega_{\mathbb{P}^{2}}(2 h), \Xi_{3}(F)\right)[-2] \\
& \cong \operatorname{RHom}_{\mathrm{D}^{\mathrm{b}}\left(\mathbb{P}^{2}\right)}\left(\Omega_{\mathbb{P}^{2}}(2 h), \Xi_{3}(F)\right)[-2],
\end{aligned}
$$

where we have used cone $\left(\mathcal{O}_{\mathbb{P}^{2}}(h)^{\oplus 5} \stackrel{\text { ev }}{\longrightarrow} \mathcal{O}_{\mathbb{P}^{2}}(h)^{\oplus 2} \oplus \mathcal{O}_{\mathbb{P}^{2}}(2 h)\right) \cong \Omega_{\mathbb{P}^{2}}(2 h)[1]$, and the first series of isomorphisms follows. 


\section{ACM BUNDLES ON CUBIC THREEFOLDS}

It remains to prove the second series of isomorphisms of the lemma. We start with the following chain of natural isomorphisms, which follows directly from the definitions:

$$
\begin{aligned}
& \operatorname{RHom}_{\mathrm{D}^{\mathrm{b}}(Y)}\left(\mathcal{O}_{Y}(-H), F\right) \cong \operatorname{RHom}_{\mathrm{D}^{\mathrm{b}}(Y)}\left(\mathcal{O}_{Y}(-H), \Xi_{3}^{-1}\left(\Xi_{3}(F)\right)\right) \\
& \cong \operatorname{RHom}_{\mathrm{D}^{\mathrm{b}}(Y)}\left(\mathcal{O}_{Y}(-H), \sigma_{*} \circ \mathbf{R}_{\mathcal{O}_{\tilde{Y}}(-h)} \circ \Phi \circ \Xi_{3}(F)\right) \\
& \cong \operatorname{RHom}_{\mathrm{D}^{\mathrm{b}}\left(\mathbb{P}^{2}, \mathcal{B}_{0}\right)}\left(\Psi \circ \mathbf{L}_{\mathcal{O}_{\widetilde{Y}}(H)}\left(\mathcal{O}_{\widetilde{Y}}(-H)\right), \Xi_{3}(F)\right) \\
& \cong \operatorname{RHom}_{\mathrm{D}^{\mathrm{b}}\left(\mathbb{P}^{2}, \mathcal{B}_{0}\right)}\left(\Psi\left(\mathcal{O}_{\widetilde{Y}}(-H)\right), \Xi_{3}(F)\right) \\
& \cong \mathrm{RHom}_{\mathrm{D}^{\mathrm{b}}\left(\mathbb{P}^{2}, \mathcal{B}_{0}\right)}\left(\mathcal{B}_{-1}, \Xi_{3}(F)\right) \text {. }
\end{aligned}
$$

The last isomorphism is an easy computation, and the lemma follows.

Now we are ready to give a geometric interpretation of the objects of $\mathfrak{N}_{d}$ (recall (2.4.3)).

Proposition 2.22. If $d=2,3$ and $G$ is a general sheaf in $\mathfrak{N}_{d}$, then the object $\Xi_{3}^{-1}(G)$ is a stable ACM bundle of rank $d$.

Proof. Again the argument can be divided in a few parts.

Step 1: The object $\Xi_{3}^{-1} G$ is a coherent sheaf. By Example 2.10, the sheaf $F_{d}$ is in $\mathbf{T}_{Y}$ and $\Xi_{3}\left(F_{d}\right) \in \mathfrak{N}_{d}$. By semi-continuity, for $G \in \mathfrak{N}_{d}$ general, the object $\Xi_{3}^{-1}(G)$ has to be a sheaf.

Step 2: First vanishing. We want to show that $H^{i}\left(Y, \Xi_{3}^{-1}(G) \otimes \mathcal{O}_{Y}(-2 H)\right)=0$ for $i=1,2$. By Lemma 2.21, we need to prove that $\operatorname{Hom}_{\mathrm{D}^{\mathrm{b}}\left(\mathbb{P}^{2}\right)}^{0}\left(\Omega_{\mathbb{P}^{2}}(2 h), G\right)=0$.

Before that, by Example 2.11, we observe that

$$
\operatorname{Hom}_{\mathrm{D}^{\mathrm{b}}\left(\mathbb{P}^{2}, \mathcal{B}_{0}\right)}^{0}\left(\mathcal{B}_{0}, \Xi_{3}\left(\mathcal{I}_{l}\right)\right)=\operatorname{Hom}_{\mathbb{P}^{2}}^{0}\left(\mathcal{O}_{\mathbb{P}^{2}}, \mathcal{O}_{l} \oplus \mathcal{O}_{l}(-1)\right) \cong \mathbb{C}
$$

and all other Hom-groups are trivial. The extension of $d$ sheaves $\Xi_{3}\left(\mathcal{I}_{l}\right)$ (with different $l$ ) lies in $\mathfrak{M}_{d}^{s}$. By semicontinuity and induction on $d$, we have

$$
\operatorname{Hom}_{\mathrm{D}^{\mathrm{b}}\left(\mathbb{P}^{2}, \mathcal{B}_{0}\right)}^{0}\left(\mathcal{B}_{0}, G\right) \cong H^{0}\left(\mathbb{P}^{2}, G\right) \cong \mathbb{C}^{d}
$$

for $G$ general in $\mathfrak{M}_{d}$. Notice that here we are implicitly using that $\chi\left(\mathcal{B}_{0}, G\right)=d$. Indeed, this follows from (2.2.3).

Case 1: Rank $d=2$. Let $G$ be supported in $j_{C}: \mathbb{P}^{1} \hookrightarrow C \in\left|\mathcal{O}_{\mathbb{P}^{2}}(2)\right|$. Let $C$ be smooth and intersect $\Delta$ transversally. Note that $\left.\Omega_{\mathbb{P}^{2}}(2 h)\right|_{C} \cong i_{*}\left(\mathcal{O}_{\mathbb{P}^{1}}(1)^{\oplus 2}\right)$, so we have to show that $H^{0}\left(\mathbb{P}^{1}, G(-1)\right)=0$. By (2.5.6) and semi-continuity, there are only two possibilities for $G$ (as an $\mathcal{O}_{\mathbb{P}^{2} \text {-module): }}$

$$
\begin{aligned}
& G \cong j_{C_{*}}\left(\mathcal{O}_{\mathbb{P}^{1}}(1) \oplus \mathcal{O}_{\mathbb{P}^{1}}(-1)\right), \\
& G \cong \mathcal{O}_{C}^{\oplus 2} .
\end{aligned}
$$

If we are in situation (2.5.8), then the desired vanishing holds.

Assume now $G \cong j_{C_{*}}\left(\mathcal{O}_{\mathbb{P}^{1}}(1) \oplus \mathcal{O}_{\mathbb{P}^{1}}(-1)\right)$. Recall that $G=\psi_{*}\left(L \otimes E_{C, 0}^{\vee}\right)$ for some $L \in \operatorname{Pic}(\widehat{C})$. We use a method from [Beau02, Ili99]. The projective bundle $\mathbb{P}\left(L \otimes E_{C, 0}^{\vee}\right) \rightarrow \widehat{C}$ corresponds by definition to the conic bundle over $\widehat{C}$ induced by the conic fibration $\pi: \widetilde{Y} \rightarrow \mathbb{P}^{2}$. More precisely, $\pi^{-1}(C)$ is a conic bundle over $C$ with ten singular fibers $\pi^{-1}(C \cap \Delta)=\bigcup_{i=1}^{10} l_{i} \cup l_{i}^{\prime}$. The lines $l_{i}$ 


\section{Martí Lahoz, Emanuele Macrì and PaOlo Stellari}

and $l_{i}^{\prime}$ are $(-1)$-curves. Then, we have

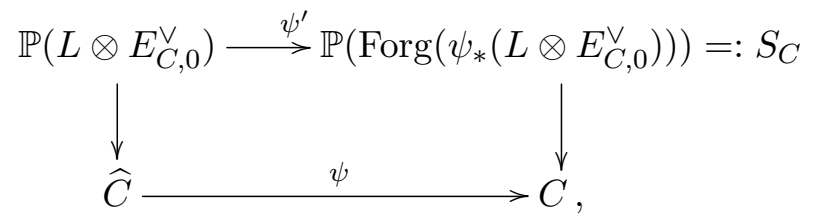

where the map $\psi^{\prime}$ factors through $\pi^{-1}(C) \rightarrow \mathbb{P}\left(\psi_{*}\left(L \otimes E_{C, 0}^{\vee}\right)\right)$, which corresponds to the blowdown of ten $(-1)$-curves, say $l_{i}$ for $i=1, \ldots 10$. The fact that $\operatorname{Forg}\left(\psi_{*}\left(L \otimes E_{C, 0}^{\vee}\right)\right)=j_{C_{*}}\left(\mathcal{O}_{\mathbb{P} 1}(1) \oplus\right.$ $\left.\mathcal{O}_{\mathbb{P}^{1}}(-1)\right)$ implies that $\mathbb{P}\left(\operatorname{Forg}\left(\psi_{*}\left(L \otimes E_{C, 0}^{\vee}\right)\right)\right)$ is isomorphic to the second Hirzebruch surface, so we have a section $c$ of $\pi^{-1}(C) \rightarrow C$ such that $c^{2}=-2$. Recall that the canonical bundle of $\tilde{Y}$ is $\mathcal{O}_{\widetilde{Y}}(-H-h)=\mathcal{O}_{\widetilde{Y}}\left(-D-S_{C}\right)$, where $D$ is the exceptional divisor. The adjunction formula gives $K_{S_{C}} \equiv-\left.D\right|_{S_{C}}$. By the adjunction formula $c^{2}=-2$ implies that $D \cdot c=0$. Hence, we can see $c$ as a rational curve in $Y$ of degree two. The space of conics in $Y$ is four-dimensional, but by Theorem $2.12, \mathfrak{M}_{2}$ has dimension five.

Case 2: Rank $d=3$. Let $G$ be supported in $C \in\left|\mathcal{O}_{\mathbb{P}^{2}}(3)\right|$. Let $C$ be smooth and intersect $\Delta$ transversally. Note that $\left.\Omega_{\mathbb{P}^{2}}(2 h)\right|_{C}=F$ is an Atiyah bundle of degree three, so we have to show that $H^{0}\left(C, G \otimes F^{\vee}\right)=0$. By (2.5.6) and semi-continuity, $G$ has only three possibilities (as $\mathcal{O}_{\mathbb{P}^{2}}$-module):

$$
\begin{aligned}
& G \cong \text { Atiyah bundle of degree three, } \\
& G \cong\left(j_{C}\right)_{*}\left(\mathcal{L}_{1} \oplus \mathcal{L}_{2}\right) \\
& G \cong\left(j_{C}\right)_{*}\left(\mathcal{L}_{0} \oplus \mathcal{L}_{3}\right)
\end{aligned}
$$

where $j_{C}$ denotes the embedding and the $\mathcal{L}_{i}$ are generic line bundles of degree $i$ on $C$.

If we are in situation (2.5.9), then the desired vanishing holds.

As before, assume for a contradiction that (2.5.10) holds. The fact that $\operatorname{Forg}\left(\psi_{*}\left(L \otimes E_{C, 0}^{\vee}\right)\right)=$ $\left(j_{C}\right)_{*}\left(\mathcal{L}_{1} \oplus \mathcal{L}_{2}\right)$ implies that $\mathbb{P}\left(\operatorname{Forg}\left(\psi_{*}\left(L \otimes E_{C, 0}^{\vee}\right)\right)\right) \rightarrow C$ has a section $c$ of $\pi^{-1}(C) \rightarrow C$ such that $c^{2}=-1$. Recall that the canonical bundle of $\tilde{Y}$ is $\mathcal{O}_{\widetilde{Y}}(-H-h)=\mathcal{O}_{\widetilde{Y}}\left(-D+h-S_{C}\right)$, where $D$ is the exceptional divisor. The adjunction formula gives $\left.K_{S_{C}} \equiv(-D+h)\right|_{S_{C}}$. By the adjunction formula $c^{2}=-1$ implies that $D \cdot c=h \cdot c-1=2$. Hence, we can see $c$ as an elliptic quintic curve in $Y$ meeting the projection line $l_{0}$ in two points. The space of elliptic quintic curves in $Y$ is ten-dimensional [MT01, Theorem 4.5], so the ones meeting $l_{0}$ in two points form an eight-dimensional family [MT01, Lemma 4.6], but $\mathfrak{M}_{3}$ has dimension ten (by Theorem 2.12).

It remains to consider the last case (2.5.11). The fact that $\operatorname{Forg}\left(\psi_{*}\left(L \otimes E_{C, 0}^{\vee}\right)\right) \cong\left(j_{C}\right)_{*}\left(\mathcal{L}_{0} \oplus \mathcal{L}_{3}\right)$ implies that $\mathbb{P}\left(\operatorname{Forg}\left(\psi_{*}\left(L \otimes E_{C, 0}^{\vee}\right)\right)\right) \rightarrow C$ has a section $c$ of $\pi^{-1}(C) \rightarrow C$ such that $c^{2}=-3$. Recall that the canonical bundle of $\widetilde{Y}$ is $\mathcal{O}_{\widetilde{Y}}(-H-h)=\mathcal{O}_{\widetilde{Y}}\left(-D+h-S_{C}\right)$, where $D$ is the exceptional divisor. The adjunction formula gives $\left.K_{S_{C}} \equiv(-D+h)\right|_{S_{C}}$. By the adjunction formula $c^{2}=-3$ implies that $D \cdot c=h \cdot c-3=0$. Hence, we can see $c$ as an elliptic curve in $Y$ of degree three (hence plane). The space of plane cubics in $Y$ is nine-dimensional, but by Theorem 2.12, $\mathfrak{M}_{3}$ has dimension ten.

Step 3: Second vanishing and stability. We want to show that $H^{1}\left(Y, \Xi_{3}^{-1}(G) \otimes \mathcal{O}_{Y}(H)\right)=0$. By the second part of Lemma 2.21, we need to prove that $H^{1}\left(\mathbb{P}^{2}, \operatorname{Forg}\left(G \otimes_{\mathcal{B}_{0}} \mathcal{B}_{1}\right)\right)=0$. Again we can argue as in Step 1 by semi-continuity and use that $G=\Xi_{3}\left(F_{d}\right)$ satisfies the vanishing. Thus 


\section{ACM BUNDLES ON CUBIC THREEFOLDS}

$\Xi_{3}^{-1}(G)$ is an ACM bundle. As observed in [CHGS12, Section 5], $\Xi_{3}^{-1}(G)$ is stable since there are no Ulrich bundles of rank one on a cubic threefold.

Note that the sheaves $\Xi_{3}^{-1}(G)$ in Proposition 2.22 are Ulrich. Since they lie in $\mathbf{T}_{Y}$, the same argument of [CHGS12, Lemma 2.4] shows that their restriction to a generic hyperplane section is again Ulrich.

To complete the non-emptiness statement of Theorem B, we should prove that there are stable Ulrich bundles for all ranks $r \geqslant 4$. For this we can use the same deformation argument as in the proof of [CHGS12, Theorem 5.7].

Remark 2.23. Note that we have also reproven that $\mathfrak{M}_{r}^{s U}$ is smooth of dimension $r^{2}+1$. Indeed, the computations $\operatorname{dim} \operatorname{Ext}^{1}(F, F)=r^{2}+1$ and $\operatorname{dim}_{\operatorname{Ext}^{2}}(F, F)=0$ have already been done in Step 1 of Theorem 2.12.

Remark 2.24. The proof above fails, for the case $d=1$, essentially only in Step 2; more precisely, the restriction $\left.\Omega_{\mathbb{P}^{2}}(2 h)\right|_{C}$ to a line $C \subset \mathbb{P}^{2}$ is not semistable.

\section{The $d=2$ case and the instanton bundles on cubic threefolds}

In this section we will describe explicitly the wall-crossing phenomena that link the space $\mathfrak{M}_{2}$ to the moduli space of semistable instanton sheaves on $Y$. This example, together with Section 2.2, should motivate our expectation that the geometry of the moduli spaces $\mathfrak{M}_{d}$ is tightly related to that of classical geometric objects associated with cubic threefolds.

The argument is a bit involved and thus we prefer to sketch it here for the convenience of the reader. First of all, we need to analyze how stability and semistability of special objects in $\mathrm{D}^{\mathrm{b}}\left(\mathbb{P}, \mathcal{B}_{0}\right)$ vary in the family of stability conditions described in Lemma 2.7 (see Section 3.1). This is conceptually rather standard but computationally a bit involved. Once this is settled, one can consider instanton sheaves $E$ and look at their images under the functor $\Xi_{3}$. It turns out that they are all stable $\mathcal{B}_{0}$-modules if $E$ is locally free (see Lemma 3.9). On the other hand, special attention has to be paid to instanton sheaves $E$ which are not locally free. The most delicate cases are when they are extensions of ideal sheaves of two lines, one of which is the line of projection $l_{0}$.

Having the toy model of $\mathfrak{M}_{1}$ in mind, it is rather clear that all this leads naturally to a wall-crossing phenomenon. This will be described in Theorem 3.10, where again we combine the classical description of the moduli space of semistable instanton sheaves [Dru00] and the machinery of (Bridgeland) stability conditions from Section 3.1.

As in Section 2.2, the approach follows closely the discussion in [MS12, Section 5], but since the corresponding numerical class is not primitive, we need some extra arguments.

\subsection{Stability}

We consider the stability function $Z_{m}$ (see Definition 2.6) and the (Bridgeland) stability condition $\sigma_{m}=\left(Z_{m}, \mathbf{A}\right)$ (see Lemma 2.7).

A (semi)stable $\mathcal{B}_{0}$-module $F \in \mathfrak{M}_{2}$ remains $\sigma_{m}$-(semi)stable for all $m>m_{0}=\frac{\sqrt{5}}{8}$. More precisely, we have the following lemma.

Lemma 3.1. Let $F \in \mathfrak{M}_{2}$. 


\section{Martí Lahoz, Emanuele Macrì and PaOlo Stellari}

(a) If $m>m_{0}=\frac{\sqrt{5}}{8}$, then $F$ is $\sigma_{m}$-stable or $F$ is the extension of two $\sigma_{m}$-stable coherent $\mathcal{B}_{0}$-modules of class $\left[\mathcal{B}_{1}\right]-\left[\mathcal{B}_{0}\right]$ (so is properly $\sigma_{m}$-semistable).

(b) If $F \in \mathfrak{N}_{2}$ and $F$ is stable, then $F$ is $\sigma_{m}$-stable for all $m>\frac{1}{4}$. If $F$ is properly semistable, then $F$ is the extension of two $\sigma_{m}$-stable coherent $\mathcal{B}_{0}$-modules of class $\left[\mathcal{B}_{1}\right]-\left[\mathcal{B}_{0}\right]$ for all $m>\frac{1}{4}$.

(c) Assume $m=m_{0}$. Then $F$ is $\sigma_{m}$-semistable and falls in one of the following cases:

(c.i) We have $F \in \mathfrak{N}_{2}$, and $F$ is $\sigma_{m_{0}}$-stable.

(c.ii) We have $F \in \mathfrak{N}_{2}$, and $F$ is properly $\sigma_{m_{0}}$-semistable and its $J H$-factors are two $\sigma_{m_{0}}$-stable coherent $\mathcal{B}_{0}$-modules of class $\left[\mathcal{B}_{1}\right]-\left[\mathcal{B}_{0}\right]$.

(c.iii) We have $F \in \mathfrak{M}_{2} \backslash \mathfrak{N}_{2}, F$ is properly $\sigma_{m_{0}}$-semistable, and its JH-factors are $\mathcal{B}_{0}[1], \mathcal{B}_{1}$, and a coherent $\mathcal{B}_{0}$-module of class of class $\left[\mathcal{B}_{1}\right]-\left[\mathcal{B}_{0}\right]$.

(c.iv) We have $F \in \mathfrak{M}_{2} \backslash \mathfrak{N}_{2}, F$ is properly $\sigma_{m_{0}}$-semistable, and its JH-factors are twice $\mathcal{B}_{0}[1]$ and twice $\mathcal{B}_{1}$.

Proof. Suppose that $0 \rightarrow A \rightarrow F \rightarrow B \rightarrow 0$ destabilizes $F$ in the stability condition $\sigma_{m}$ for $m>\frac{1}{4}$, where $A, B \in \mathbf{A}$ and $A$ is $\sigma_{m}$-stable. We have

$$
0 \rightarrow \mathcal{H}^{-1}(B) \rightarrow A \rightarrow F \rightarrow \mathcal{H}^{0}(B) \rightarrow 0
$$

and $\operatorname{Im}\left(Z_{m}([F])\right)=4 m$. Note that $J_{m}:=\operatorname{Im}\left(Z_{m}\right)$ is an additive function in $K\left(\mathbf{C o h}\left(\mathbb{P}^{2}, \mathcal{B}_{0}\right)\right)$ that takes values in $m \mathbb{Z}$. Moreover, by the main property of the stability function since $A, B \in \mathbf{A}$, we have $J_{m}(A), J_{m}(B) \geqslant 0$. Thus, $J_{m}(A)$ can only take values in $\{0, m, 2 m, 3 m, 4 m\}$. Note also that, since $F$ and $\mathcal{H}^{0}(B)$ are torsion, $\operatorname{rk}\left(\mathcal{H}^{-1}(B)\right)=\operatorname{rk}(A)$.

Let $\operatorname{ch}([A])=\left(r, c_{1}, \operatorname{ch}_{2}\right)$. Observe that $\operatorname{Re} Z_{m}(F)=0$, hence $A$ destabilizes if $\operatorname{Re} Z_{m}(A) \leqslant 0$. Since $A$ is $\sigma_{m}$-stable, we distinguish two cases: either $A$ is torsion or $A$ is torsion-free of rank $r=\operatorname{rk}(A)=\operatorname{rk}\left(\mathcal{H}^{-1}(B)\right)>0$.

If $A$ is torsion, then $\operatorname{rk}\left(\mathcal{H}^{-1}(B)\right)=0$ and since $\mathcal{H}^{-1}(B) \in \mathbf{F}$, we have $\mathcal{H}^{-1}(B)=0$. By [BMMS12, Lemma 2.13(ii)], we have that $c_{1}$ is even. As $F \in \mathfrak{M}_{2}$ is a semistable $\mathcal{B}_{0}$-module, $c_{1} \leqslant 2$. In that case, $A$ cannot be supported on points ( $F$ is locally free on its support), so we have $c_{1}=2$. In order to destabilize $F$ in the stability condition $\sigma_{m}$, we need

$$
\operatorname{Re}\left(Z_{m}([A])\right)=r m^{2}-\frac{9 r}{64}-\frac{c_{1}}{2}-\frac{\mathrm{ch}_{2}}{2} \leqslant 0,
$$

so $\operatorname{ch}_{2} \geqslant-2$. But $F \in \mathfrak{M}_{2}$ and since $c_{1}=2$, we have $\operatorname{ch}_{2} \leqslant-2$. Thus $\operatorname{ch}([A])=(0,2,-2)$ and $F$ is a properly $\sigma_{m}$-semistable object (for all $m>\frac{1}{4}$ ) whose JH-factors have class $\left[\mathcal{B}_{1}\right]-\left[\mathcal{B}_{0}\right]$.

Suppose now that $A \in \mathbf{T}$ is torsion-free, so all its HN-factors with respect to the slope stability have slope $\mu>-1$. Note that $J_{m}(A)>0$ since $A$ cannot be supported on points. Moreover, if $J_{m}(A)=4 m$, then $J_{m}(B)=0, \phi(B)=1$, and $B$ would not destabilize $F$. So $J_{m}(A) \in\{m, 2 m, 3 m\}$.

In order to $\sigma_{m}$-destabilize $F$, we need

$$
\operatorname{Re}\left(Z_{m}([A])\right)=r m^{2}-\frac{9 r}{64}-\frac{c_{1}}{2}-\frac{\mathrm{ch}_{2}}{2}=\frac{1}{r}\left(-\chi(A, A)+m^{2} r^{2}-\frac{1}{4}\left(r+c_{1}\right)^{2}\right) \leqslant 0 .
$$

Moreover, we can assume that $A$ is $\mu$-stable. Then, by Lemma 2.4 , we have $-1 \leqslant-\chi(A, A)$. Since $m \leqslant J_{m}(A) \leqslant 3 m$, we have $-\frac{9}{4} \leqslant-\frac{1}{4}\left(r+c_{1}\right)^{2}$. Thus, from (3.1.1) and the previous inequalities we deduce $m^{2} r^{2}-\frac{13}{4} \leqslant 0$. Since $m>\frac{1}{4}$ and $r \in 4 \mathbb{N}_{>0}$, this implies $r=4$.

Now we go through a case by case study depending on $J_{m}(A)$. 


\section{ACM BUNDLES ON CUBIC THREEFOLDS}

Case $J_{m}(A)=m$. In this case $c_{1}=-3$ and $r=4$, so (3.1.1) becomes $-\chi(A, A)+16 m^{2}-\frac{1}{4} \leqslant 0$. Since $m>\frac{1}{4}$ and $-\chi(A, A)=\operatorname{hom}^{1}(A, A)-1$, we have $\operatorname{hom}^{1}(A, A)-\frac{1}{4}<0$, so $A$ is rigid. Moreover, $\operatorname{ch}_{2}=\frac{5}{2}$, by $(2.2 .2)$. Hence, $A \cong \mathcal{B}_{1}$ and $[B]=\left[\mathcal{B}_{1}\right]-2\left[\mathcal{B}_{0}\right]$. Moreover, since $16 m^{2}-\frac{5}{4} \leqslant 0$, we have $m \leqslant \frac{\sqrt{5}}{8}$.

Case $J_{m}(A)=2 m$. Under this assumption $c_{1}=-2$, which is impossible by [BMMS12, Lemma $2.13($ ii)].

Case $J_{m}(A)=3 m$. Here $c_{1}=-1$ and $r=4$, so (3.1.1) becomes $-\chi(A, A)+16 m^{2}-\frac{9}{4} \leqslant 0$. Since $m>\frac{1}{4}$ and $-\chi(A, A)=\operatorname{hom}^{1}(A, A)-1$, we have $\operatorname{hom}^{1}(A, A)-\frac{9}{4}<0$, which implies $\operatorname{hom}^{1}(A, A) \leqslant 2$.

(a) If $A$ is rigid, then $\operatorname{ch}_{2}=\frac{3}{2}$, by (2.2.2). Hence $A \cong \mathcal{B}_{2}$, which is impossible since $0 \neq$ $\operatorname{hom}\left(\mathcal{B}_{2}, F\right)=h^{0}\left(\mathbb{P}^{2}, F(-h)\right)$ contradicts the Gieseker semistability of $F \in \mathfrak{M}_{2}$.

(b) If $\chi(A, A)=0$, then $\operatorname{ch}_{2}=1$, by (2.2.2). This implies that $[A] \notin \mathbb{Z}\left[\mathcal{B}_{-1}\right] \oplus \mathbb{Z}\left[\mathcal{B}_{0}\right] \oplus \mathbb{Z}\left[\mathcal{B}_{1}\right]$, which contradicts [BMMS12, Proposition 2.12].

(c) If $\chi(A, A)=-1$, then $\operatorname{ch}_{2}=\frac{1}{2}$. Hence $[A]=-\left[\mathcal{B}_{0}\right]+2\left[\mathcal{B}_{1}\right]$, so $B \cong \mathcal{B}_{0}[1]$. In particular, $\mathcal{H}^{0}(B)=0$. Moreover, $16 m^{2}-\frac{5}{4} \leqslant 0$, so $m \leqslant \frac{\sqrt{5}}{8}$.

Summarizing, if $F \in \mathfrak{N}_{2}$ is stable, then it is $\sigma_{m}$-stable for all $m>\frac{1}{4}$. If $F \in \mathfrak{N}_{2}$ is properly semistable, then its two JH-factors are $\sigma_{m}$-stable for all $m>\frac{1}{4}$. If $F \in \mathfrak{M}_{2} \backslash \mathfrak{N}_{2}$, since $\chi\left(\mathcal{B}_{1}, F\right)=0$ we have

$$
\operatorname{hom}\left(\mathcal{B}_{1}, F\right)=\operatorname{hom}^{1}\left(\mathcal{B}_{1}, F\right)=\operatorname{hom}^{1}\left(F, \mathcal{B}_{0}\right)=\operatorname{hom}\left(F, \mathcal{B}_{0}[1]\right),
$$

and $F$ admits a morphism from $\mathcal{B}_{1}$ and also has a morphism to $\mathcal{B}_{0}[1]$. Hence it could be $J_{m}(A) \in$ $\{m, 3 m\}$.

We study more precisely these two cases. If $J_{m}(A)=m$, then we claim that there exist the following exact sequences in A:

$$
\begin{array}{r}
0 \rightarrow \mathcal{B}_{1} \rightarrow F \rightarrow C \rightarrow 0, \quad \text { where }[C]=\left[\mathcal{B}_{1}\right]-2\left[\mathcal{B}_{0}\right], \\
0 \rightarrow C^{\prime} \rightarrow F \rightarrow \mathcal{B}_{0}[1] \rightarrow 0, \quad \text { where }\left[C^{\prime}\right]=2\left[\mathcal{B}_{1}\right]-\left[\mathcal{B}_{0}\right] .
\end{array}
$$

The second exact sequence is obtained from the first one using $\chi\left(\mathcal{B}_{1}, F\right)=0$, so $\operatorname{hom}_{\mathbf{A}}\left(F, \mathcal{B}_{0}[1]\right) \neq$ 0 . Indeed, it remains to prove that $F \rightarrow \mathcal{B}_{0}[1]$ needs to be surjective in $\mathbf{A}$. If not, let $L$ be the cokernel. Clearly $\mathcal{H}^{0}(L)=0$. Then $L=L^{\prime}[1]$, where $L^{\prime}$ is a torsion-free $\mathcal{B}_{0}$-module in $\mathbf{F}$. Let $T:=\operatorname{Im}_{\mathbf{A}}\left(F \rightarrow B_{0}[1]\right)$. Note that $\mathcal{H}^{0}(T)$ is a torsion sheaf. Then, if $L^{\prime} \neq 0, \mathcal{B}_{0} \rightarrow L^{\prime}$ needs to be injective and $\mathcal{H}^{-1}(T)=0$. Therefore $T \in \mathbf{T}$ and it is a quotient of $F$ as a $\mathcal{B}_{0}$-module. We know that $F$ is a Gieseker semistable $\mathcal{B}_{0}$-module and $c_{1}(T) \geqslant 2$. This implies $c_{1}\left(L^{\prime}\right) \geqslant-3$, which contradicts $L^{\prime} \in \mathbf{F}$, since by [BMMS12, Lemma 2.13(i)] $\operatorname{rk}\left(L^{\prime}\right) \geqslant 4$.

Equivalently, if we are in the case $J_{m}(A)=3 m$, then we claim that we get again the exact sequences (3.1.2). Indeed, now the first exact sequence is obtained from the second one by using $\chi\left(\mathcal{B}_{1}, F\right)=0$, so $\operatorname{hom}_{\mathbf{A}}\left(\mathcal{B}_{1}, F\right) \neq 0$. In that case we need to prove that $B_{1} \rightarrow F$ is injective in $\mathbf{A}$. If it is not, let $K$ be the kernel. Clearly $\mathcal{H}^{-1}(K)=0$. Then $K$ is a $\mathcal{B}_{0}$-module in $\mathbf{T}$. If $K \neq 0$, then $K \rightarrow \mathcal{B}_{1}$ needs to be injective. Hence $T:=\operatorname{Im}_{\mathbf{A}}\left(\mathcal{B}_{1} \rightarrow F\right) \in \mathbf{T}$ and it is a subobject of $F$ as a $\mathcal{B}_{0}$-module. We know that $F$ is a Gieseker semistable $\mathcal{B}_{0}$-module and $c_{1}(T) \leqslant 2$. This implies $c_{1}(K) \leqslant-5$, which contradicts the fact that $K \in \mathbf{T}$, since by [BMMS12, Lemma 2.13(i)] $\operatorname{rk}(K) \geqslant 4$.

In both cases we can summarize the situation in the following commutative diagram of exact 
sequences of $\sigma_{m_{0}}$-semistable objects in $\mathbf{A}$ :

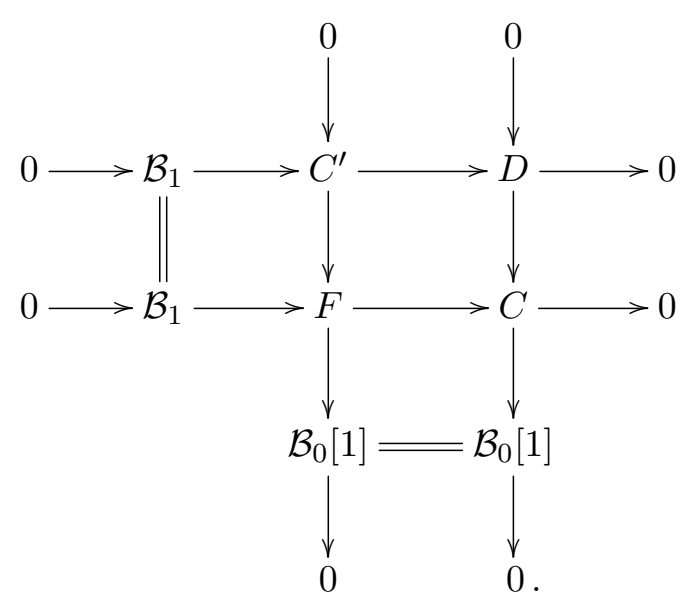

On the one hand, by the middle vertical exact sequence, we have $\mathcal{H}^{-1}\left(C^{\prime}\right)=0$. Hence, by the top horizontal exact sequence, $\mathcal{H}^{-1}(D)=0$ or $\mathcal{H}^{-1}(D)=\mathcal{B}_{1}$. Note that $\mathcal{H}^{-1}(D)=\mathcal{B}_{1} \notin \mathbf{F}$, so $D \in \operatorname{Coh}\left(\mathbb{P}^{2}, \mathcal{B}_{0}\right)$.

Summing up, when $m=m_{0}$, we have in those cases that $\mathcal{B}_{0}[1]$ and $\mathcal{B}_{1}$ are two JH-factors of $F$. Moreover, the remaining part $D$ of the JH-filtration is a $\sigma_{m_{0}}$-semistable $\mathcal{B}_{0}$-module, with class $\left[\mathcal{B}_{1}\right]-\left[\mathcal{B}_{0}\right]$.

When $C \in\left\langle\mathcal{B}_{1}\right\rangle^{\perp}$ (equivalently, $D \in \mathfrak{N}_{1}$ ), we fall into case (c.iii) and the JH-factors for $m=m_{0}$ are $\mathcal{B}_{0}[1], \mathcal{B}_{1}$, and $D$. When $C \notin\left\langle\mathcal{B}_{1}\right\rangle^{\perp}$ (equivalently, $D \in \mathfrak{M}_{1} \backslash \mathfrak{N}_{1}$ ), we fall into case (c.iv).

Let $w$ be the numerical class $2\left[\mathcal{B}_{1}\right]-2\left[\mathcal{B}_{0}\right]$. As a consequence of the previous lemma we get that $\mathfrak{M}_{2}$ embeds inside $\mathfrak{M}^{\sigma_{m}}\left(\mathbb{P}^{2}, \mathcal{B}_{0} ; w\right)$ when $m>m_{0}$. With the aim of proving the other inclusion, we need the following lemma, which adapts [MS12, Lemma 5.8] to our situation.

LemMa 3.2. Let $m_{1}>m_{0}=\frac{\sqrt{5}}{8}$ and let $G$ be a $\sigma_{m_{1}}$-(semi)stable object with numerical class $w$. Then $G$ is $\sigma_{m}$-(semi)stable for all $m>m_{0}$ and $\sigma_{m_{0}}$-semistable.

Proof. Assume, for a contradiction, that $G$ is not $\sigma_{m}$-semistable (respectively, $\sigma_{m}$-stable) for $m \geqslant m_{0}$. Then we have an exact sequence in $\mathbf{A}$

$$
0 \rightarrow A \rightarrow G \rightarrow B \rightarrow 0
$$

where $A \neq 0$ is $\sigma_{m}$-stable and $\operatorname{Re}\left(Z_{m}(A)\right)<0$ (respectively, $\operatorname{Re}\left(Z_{m}(A)\right) \leqslant 0$ ). Let $\operatorname{ch}(A)=$ $\left(r, c_{1}, \mathrm{ch}_{2}\right)$. The same argument as in [MS12, Lemma 5.8] shows that $r \neq 0$ and $\operatorname{Im}\left(Z_{m}(A)\right) \in$ $\{m, 2 m, 3 m\}$. But then, the same casuistry as in Lemma 3.1 shows that this can only happen when $m<m_{0}$ (respectively, $m<m_{0}$ or $m=m_{0}$ and $G \notin\left\langle\mathcal{B}_{1}\right\rangle^{\perp}$ ).

Now, let $G \in \mathfrak{M}^{\sigma_{m_{1}}}\left(\mathbb{P}^{2}, \mathcal{B}_{0} ; w\right)$ be a (semi)stable object for $m_{1} \geqslant m_{0}$. By Lemma 3.2, we have two possibilities: either $G$ is $\sigma_{m}$-(semi)stable for all $m \geqslant m_{0}$, or $m_{1}=m_{0}$ and $G$ either stabilizes or destabilizes for all $m>m_{0}$. We will see in Lemma 3.5 that it destabilizes or stabilizes depending on whether $\operatorname{hom}\left(\mathcal{B}_{1}, \mathcal{G}\right)$ is maximal in its S-equivalence class or not.

Lemma 3.3. Let $G \in \mathbf{A}$ be a $\sigma_{m}$-(semi)stable object for all $m \geqslant m_{0}$, with numerical class $w$. Then $G$ is a (semi)stable $\mathcal{B}_{0}$-module, pure of dimension one. 


\section{ACM BUNDLES ON CUBIC THREEFOLDS}

Proof. We argue as in [MS12, Lemma 5.9] to deduce that $G$ is a pure $\mathcal{B}_{0}$-module of dimension one. If $A$ is a stable $\mathcal{B}_{0}$-module that destabilizes $G$, then $\operatorname{Re} Z_{m}(A) \leqslant 0$ (respectively, $<0$ ), so $G$ would not be $\sigma_{m^{-}}$(semi)stable.

As a straightforward consequence of the previous lemmas, we get the following.

Corollary 3.4. Let $w=2\left[\mathcal{B}_{1}\right]-2\left[\mathcal{B}_{0}\right]$. Then $\mathfrak{M}_{2}=\mathfrak{M}^{\sigma_{m}}\left(\mathbb{P}^{2}, \mathcal{B}_{0} ; w\right)$ for all $m>m_{0}=\frac{\sqrt{5}}{8}$.

Finally, we study in general the S-equivalence classes in $\mathfrak{M}^{\sigma_{m_{0}}}\left(\mathbb{P}^{2}, \mathcal{B}_{0} ; w\right)$ which contain objects outside $\left\langle\mathcal{B}_{1}\right\rangle^{\perp}$. In particular, we will study the S-equivalence classes of the objects $F \in \mathfrak{M}_{2}$, which become $\sigma_{m_{0}}$-semistable with JH-factors as in cases (c.iii) and (c.iv) of Lemma 3.1. The following lemma will be useful in the next section to prove Theorem 3.10.

Lemma 3.5. Let $w=2\left[\mathcal{B}_{1}\right]-2\left[\mathcal{B}_{0}\right]$. An object $G$ in $\mathfrak{M}^{\sigma_{m_{0}}}\left(\mathbb{P}^{2}, \mathcal{B}_{0} ; w\right) \backslash\left\langle\mathcal{B}_{1}\right\rangle^{\perp}$ falls into one of the following cases:

(a) The object $G$ is in the $S$-equivalence class of $\mathcal{B}_{0}[1] \oplus \mathcal{B}_{1} \oplus \Xi_{3}\left(\mathcal{I}_{l}\right)$, with $l \neq l_{0}$. The indecomposable objects in this $S$-equivalence class in $\mathfrak{M}^{\sigma_{m}}\left(\mathbb{P}^{2}, \mathcal{B}_{0} ; w\right)$ are represented by:

(a.i) Gieseker semistable $\mathcal{B}_{0}$-modules in $\mathfrak{M}_{2} \backslash \mathfrak{N}_{2}$ that are parametrized by a $\mathbb{P}^{2}$;

(a.ii) Gieseker properly semistable $\mathcal{B}_{0}$-modules in $\mathfrak{M}_{2} \backslash \mathfrak{N}_{2}$ that are parametrized by a $\mathbb{P}^{1}$ contained in the $\mathbb{P}^{2}$ above; in the complement $\mathbb{P}^{1}$ inside $\mathbb{P}^{2}$, the $\mathcal{B}_{0}$-modules are Gieseker stable;

(a.iii) an extension of $\Xi_{3}\left(\mathcal{I}_{l_{0}}\right)$ and $\Xi_{3}\left(\mathcal{I}_{l}\right)$, which lies in $\left\langle\mathcal{B}_{1}\right\rangle^{\perp}$.

(b) The object $G$ is in the $S$-equivalence class of $\mathcal{B}_{0}^{\oplus 2}[1] \oplus \mathcal{B}_{1}^{\oplus 2}$. The indecomposable objects in this $S$-equivalence class in $\mathfrak{M}^{\sigma_{m_{0}}}\left(\mathbb{P}^{2}, \mathcal{B}_{0} ; w\right)$ are represented by:

(b.i) Gieseker properly semistable $\mathcal{B}_{0}$-modules $G \in \mathfrak{M}_{2} \backslash \mathfrak{N}_{2}$; they have $\operatorname{hom}\left(\mathcal{B}_{1}, G\right)=2$, their $S$-equivalence classes as $\mathcal{B}_{0}$-modules are parametrized by a $\mathbb{P}^{2}$, and each $S$-equivalence class is $\mathbb{C}^{2}$;

(b.ii) indecomposable extensions of $\Xi_{3}\left(\mathcal{I}_{l_{0}}\right)$ with itself, which are then in $\left\langle\mathcal{B}_{1}\right\rangle^{\perp}$;

(b.iii) objects $G$ such that $\operatorname{hom}\left(\mathcal{B}_{1}, G\right)=1$.

These are the only $S$-equivalence classes that contain $\sigma_{m_{0}}$-semistable objects that get properly destabilized for $m>m_{0}$ and $m<m_{0}$.

Proof. If $G \notin\left\langle\mathcal{B}_{1}\right\rangle^{\perp}$, then $\mathcal{B}_{1} \rightarrow G$ is necessarily an injection in $\mathbf{A}$. Indeed, let $T:=\operatorname{Im}_{\mathbf{A}}\left(\mathcal{B}_{1} \rightarrow\right.$ $F)$. Since $\mathcal{B}_{1}$ is $\sigma_{m_{0}}$-stable, with $\operatorname{Re}\left(Z_{m_{0}}\left(\mathcal{B}_{1}\right)\right)=0$, if $T \neq \mathcal{B}_{1}$, then $\operatorname{Re}\left(Z_{m_{0}}(T)\right)>0$. This contradicts the semistability of $G$. Thus, $\mathcal{B}_{1}$ is a JH-factor of $G$. Since $\chi\left(\mathcal{B}_{1}, G\right)=0$, we have $\operatorname{hom}\left(\mathcal{B}_{1}, G\right)=\operatorname{hom}\left(G, \mathcal{B}_{0}[1]\right)$. The same argument shows that $G \rightarrow \mathcal{B}_{0}[1]$ is necessarily a surjection in A. Thus, $\mathcal{B}_{0}[1]$ is another JH-factor of $G$.

Hence, as in the proof of Lemma 3.1, $G$ necessarily sits in the commutative diagram (3.1.3). Note that $D \in \mathbf{A}$, but unlike in Lemma $3.1, D$ is not necessarily in $\mathbf{C o h}\left(\mathbb{P}^{2}, \mathcal{B}_{0}\right)$, because we have not assumed that $G$ is in $\operatorname{Coh}\left(\mathbb{P}^{2}, \mathcal{B}_{0}\right)$.

If $D \in\left\langle\mathcal{B}_{1}\right\rangle^{\perp}$, then $D \cong \Xi_{3}\left(\mathcal{I}_{l}\right)$ for some line $l$, by [BMMS12, Theorem 4.1]. Note that $D \in \mathbf{C o h}\left(\mathbb{P}^{2}, \mathcal{B}_{0}\right)$ if and only if $l \neq l_{0}$ and then, we are in case (a). If $D \notin\left\langle\mathcal{B}_{1}\right\rangle^{\perp}$ or $l=l_{0}$, then $D$ is still properly $\sigma_{m_{0}}$-semistable, with JH-factors $\mathcal{B}_{0}[1]$ and $\mathcal{B}_{1}$ and we are in case (b).

Suppose that we are in case (a) and let $G$ be a representative in the S-equivalence class such that $\operatorname{hom}\left(\mathcal{B}_{1}, G\right) \neq 0$. Note that an element in $\operatorname{Hom}^{1}\left(\mathcal{B}_{0}[1], \mathcal{B}_{1}\right)$ corresponds to an element in the projective line $\mathbb{P}^{1}$ which is the exceptional locus of the map $\mathfrak{M}_{1} \rightarrow F(Y)$ described in Proposition 


\section{Martí Lahoz, Emanuele Macrì and PaOlo Stellari}

2.13. Taking the unique non-trivial extension of one of these $\mathcal{B}_{0}$-modules with $\Xi_{3}\left(\mathcal{I}_{l}\right)$, we obtain a $\mathbb{P}^{1}$ of properly semistable $\mathcal{B}_{0}$-modules (this is case (a.ii)).

Now we start with an element in $\operatorname{Hom}^{1}\left(\Xi_{3}\left(\mathcal{I}_{l}\right), \mathcal{B}_{1}\right)$. By Example 2.11, we have

$$
\operatorname{hom}^{1}\left(\Xi_{3}\left(\mathcal{I}_{l}\right), \mathcal{B}_{1}\right)=h^{1}\left(\mathbb{P}^{1}, \mathcal{O}_{\mathbb{P}^{1}}(-1) \oplus \mathcal{O}_{\mathbb{P}^{1}}(-2)\right)=1 \text {. }
$$

Let $C^{\prime} \in \operatorname{Hom}^{1}\left(\Xi_{3}\left(\mathcal{I}_{l}\right), \mathcal{B}_{1}\right)$. Clearly $C^{\prime} \in \mathbf{C o h}\left(\mathbb{P}^{2}, \mathcal{B}_{0}\right)$ since $\Xi_{3}\left(\mathcal{I}_{l}\right)$ and $\mathcal{B}_{1}$ are also elements of $\operatorname{Coh}\left(\mathbb{P}^{2}, \mathcal{B}_{0}\right)$.

Note that $\operatorname{hom}^{1}\left(\mathcal{B}_{0}[1], C^{\prime}\right)=3$, because

$$
0 \rightarrow \operatorname{Hom}\left(\mathcal{B}_{0}, \mathcal{B}_{1}\right) \rightarrow \operatorname{Hom}^{1}\left(\mathcal{B}_{0}[1], C^{\prime}\right) \rightarrow \operatorname{Hom}\left(\mathcal{B}_{0}, \Xi_{3}\left(\mathcal{I}_{l}\right)\right) \rightarrow 0 .
$$

Let $G \in \operatorname{Hom}^{1}\left(\mathcal{B}_{0}[1], C^{\prime}\right)$. We want to see that $G$ is a $\mathcal{B}_{0}$-module. We have

$$
0 \rightarrow \mathcal{H}^{-1}(G) \rightarrow \mathcal{B}_{0} \rightarrow C^{\prime} \rightarrow \mathcal{H}^{0}(G) \rightarrow 0 .
$$

Since $\mathcal{B}_{0}$ is torsion-free and $\operatorname{rk}\left(C^{\prime}\right)=\operatorname{rk}\left(\mathcal{B}_{0}\right)$, either $\mathcal{B}_{0} \rightarrow \mathcal{C}^{\prime}$ is zero, or $\mathcal{H}^{-1}(G)=0$. Hence, the non-trivial extensions between $\mathcal{B}_{0}[1]$ and $C^{\prime}$ are $\mathcal{B}_{0}$-modules and they are parametrized by a $\mathbb{P}^{2}$. When the first extension is trivial, that is, $C^{\prime}=\mathcal{B}_{1} \oplus \Xi_{3}\left(\mathcal{I}_{l}\right)$, we recover the previous case.

Finally, we want to see that these extensions $G$ are Gieseker semistable $\mathcal{B}_{0}$-modules. Since $G$ is $\sigma_{m_{0}}$-semistable, up to choosing $\varepsilon$ small enough, $G$ is $\sigma_{m}$-semistable for all $m \in\left(m_{0}, m_{0}+\varepsilon\right)$. Indeed, if not, by [Bri08, Proposition 9.3], the HN-factors of $G$ in the stability condition $\sigma_{m}$, for $m \in\left(m_{0}, m_{0}+\varepsilon\right)$, would survive in the stability condition $\sigma_{m_{0}}$. This would contradict the $\sigma_{m_{0}}$-semistability of $G$. Since we have seen that $\mathfrak{M}_{2}=\mathfrak{M}^{\sigma_{m}}\left(\mathbb{P}^{2}, \mathcal{B}_{0} ; w\right)$ for all $m>m_{0}$, we get that $G \in \mathfrak{M}_{2}$ and thus case (a.i). If $G$ is properly semistable, then $G$ is the extension of two stable $\mathcal{B}_{0}$-modules, $G_{1}$ and $G_{2}$. Since $\operatorname{hom}\left(\mathcal{B}_{1}, G\right) \neq 0$, we can suppose that $G_{1} \in \mathfrak{N}_{1}$ and $G_{2} \in \mathfrak{M}_{1} \backslash \mathfrak{N}_{1}$, and we are in the aforementioned $\mathbb{P}^{1}$.

Now, suppose that we are in case (a) and let $G$ be a representative in the S-equivalence class in $\left\langle\mathcal{B}_{1}\right\rangle^{\perp}$. Since $\operatorname{hom}^{1}\left(\mathcal{B}_{1}, \Xi_{3}\left(\mathcal{I}_{l}\right)\right)=0$, we need to start with an element in $\operatorname{Hom}^{1}\left(\mathcal{B}_{1}, \mathcal{B}_{0}[1]\right)$. By [BMMS12, Exercise 2.11], the only non-trivial extension in $\operatorname{Hom}^{1}\left(\mathcal{B}_{1}, \mathcal{B}_{0}[1]\right)$ is $\Xi_{3}\left(\mathcal{I}_{l_{0}}\right)$ and we get case (a.iii). Thus we conclude the analysis of case (a).

Suppose that we are in case (b) and let $G$ be a representative in the S-equivalence class such that $\operatorname{hom}\left(\mathcal{B}_{1}, G\right)=2$. By the same argument as before, an extension $C$ of $\mathcal{B}_{1}$ with itself needs to be a subobject of $G$ in $\mathbf{A}$ while an extension $C^{\prime}$ of $\mathcal{B}_{0}[1]$ with itself is a quotient of $G$ in A. Note that necessarily $C=\mathcal{B}_{1}^{\oplus 2}$ and $C^{\prime}=\mathcal{B}_{0}^{\oplus 2}[1]$. Hence we consider an element in $G \in \operatorname{Hom}^{1}\left(\mathcal{B}_{0}^{\oplus 2}[1], \mathcal{B}_{1}^{\oplus 2}\right)$.

Equivalently we can construct $G$ as the extension of two sheaves $G_{1}$ and $G_{2}$ in the exceptional locus of the map $\mathfrak{M}_{1} \rightarrow F(Y)$ described in Proposition 2.13. Each of them is parametrized by a $\mathbb{P}^{1}$. But since the roles of $G_{1}$ and $G_{2}$ are symmetric, we obtain that the S-equivalence classes of the $G$ as objects in $\mathfrak{M}_{2}$ are parametrized by $\mathbb{P}^{1} \times \mathbb{P}^{1}$ quotiented by the natural involution. Thus, the S-equivalence classes of the objects $G$ are parametrized by a $\mathbb{P}^{2}$ and we obtain case (b.i). Note that $\operatorname{Ext}^{1}\left(G_{1}, G_{2}\right) \cong \mathbb{C}^{2}$.

Let $G$ be in $\left\langle\mathcal{B}_{1}\right\rangle^{\perp}$ and suppose that we are in case (b). Again, $G$ is obtained from an element in $\operatorname{Hom}^{1}\left(\mathcal{B}_{1}^{\oplus 2}, \mathcal{B}_{0}^{\oplus 2}[1]\right)$. Equivalently, we can construct $G$ as the extension of the two unique nontrivial extensions in $\operatorname{Hom}^{1}\left(\mathcal{B}_{1}, \mathcal{B}_{0}[1]\right)$. Each of them is $\Xi_{3}\left(\mathcal{I}_{l_{0}}\right)$ and $\operatorname{Ext}^{1}\left(\Xi_{3}\left(\mathcal{I}_{l_{0}}\right), \Xi_{3}\left(\mathcal{I}_{l_{0}}\right)\right) \cong \mathbb{C}^{2}$. This is case (b.ii)

The remaining indecomposable objects $G$ in case (b) have $\operatorname{hom}\left(\mathcal{B}_{1}, G\right)=1$ (as in (b.iii)) and the last statement of the lemma follows from the fact that these are the only S-equivalence 


\section{ACM BUNDLES ON CUBIC THREEFOLDS}

classes that contain the objects $G$ such that $\operatorname{hom}\left(\mathcal{B}_{1}, G\right) \neq 0$ and the objects $G^{\prime}$ such that $\operatorname{hom}\left(G^{\prime}, \mathcal{B}_{1}\right) \neq 0$.

Remark 3.6. Notice that the S-equivalence classes in cases (a) and (b) contain the $\mathcal{B}_{0}$-modules in cases (c.iii) and (c.iv) of Lemma 3.1, respectively. Moreover, from Lemma 3.5 and [Bri08, Proposition 9.3], it follows that a $\sigma_{m_{0}}$-semistable object $G$ remains semistable for $\sigma_{m}$ with $m>$ $m_{0}$ if and only if $\operatorname{hom}\left(\mathcal{B}_{1}, G\right)$ is maximal in its S-equivalence class. This happens in cases (a.i) and (b.i) of the previous lemma. Under these circumstances, two objects $G_{1}$ and $G_{2}$ in the same S-equivalence class in $\sigma_{m_{0}}$ belong to different S-equivalence classes in $\sigma_{m}$ for $m>m_{0}$. For this, one uses that $G_{1}$ and $G_{2}$ are Gieseker (semi)stable and invokes Corollary 3.4. On the other hand, $G$ remains semistable for $\sigma_{m}$ with $m<m_{0}$ if $\operatorname{hom}\left(\mathcal{B}_{1}, G\right)=0$. This happens in cases (a.iii) and (b.ii) of Lemma 3.5. It is clear that if $G$ is as in case (b.iii), then $G$ is not $\sigma_{m}$-semistable for $m>m_{0}$ or $\frac{1}{4}<m<m_{0}$.

\subsection{Instanton sheaves}

Now we want to give a geometric interpretation of $\mathfrak{M}^{\sigma_{m}}\left(\mathbb{P}^{2}, \mathcal{B}_{0} ; w\right)$ for $m \leqslant m_{0}$. The appropriate objects are the instanton sheaves.

Definition 3.7. We say that $E \in \mathbf{C o h}(Y)$ is an instanton sheaf if $E$ is a Gieseker semistable sheaf of rank two and Chern classes $c_{1}(E)=0$ and $c_{2}(E)=2$. When $E$ is locally free, we call it an instanton bundle.

An instanton sheaf according to the definition above would be called an instanton sheaf of charge two in the existing literature. In general, an instanton bundle of charge $s \geqslant 2$ is a locally free sheaf $E$ of rank two with Chern classes $c_{1}(E)=0$ and $c_{2}(E)=s$, and such that $H^{1}(Y, E(-1))=0$ (see, for example, [Kuz04, Definition 2.4]). It is easy to show that if the charge is minimal (that is, $c_{2}(E)=2$ ), then the condition $H^{1}(Y, E(-1))=0$ is automatically satisfied (see [Kuz12, Corollary 3.3]).

Remark 3.8. By [Dru00, Theorem 3.5], each semistable instanton sheaf falls under one of the following cases:

(1) The sheaf $E$ is stable and locally free.

(2) The sheaf $E$ is stable but not locally free. In this case, $E$ is obtained by the construction in Example 2.10. In fact, these are the only stable instanton sheaves that are not locally free.

(3) The sheaf $E$ is properly semistable. In this situation, $E$ is extension of two ideal sheaves of lines in $Y$.

Moreover, given a stable instanton bundle $E$, the bundle $E(1)$ is globally generated [Dru00, Theorem 2.4], so $E$ is an Ulrich bundle. Indeed, $E$ is associated with a non-degenerate smooth elliptic quintic $C$ via the Serre construction (see [Dru00, Corollary 2.6] and compare it with Lemma 2.19).

The following will be crucial in our analysis.

Lemma 3.9. Let $E$ be a stable instanton bundle. Then $\Xi_{3}(E)$ is a stable $\mathcal{B}_{0}$-module.

Proof. Let $F$ be a stable instanton bundle of minimal charge. By [Dru00, Corollary 2.6] a stable Ulrich bundle $F$ of rank two is associated with a non-degenerate smooth elliptic quintic $C$ via the Serre construction

$$
0 \rightarrow \mathcal{O}_{Y}(-H) \rightarrow F \rightarrow \mathcal{I}_{C}(H) \rightarrow 0
$$




\section{Martí Lahoz, Emanuele Macrì and PaOlo Stellari}

Note that $\Psi\left(\sigma^{*} \mathcal{O}_{Y}(-H)\right)=\mathcal{B}_{-1}$ and $\Psi\left(\sigma^{*} \mathcal{O}_{Y}(H)\right)=\mathcal{B}_{2}[1]$. Applying the functor $\Psi \circ \sigma^{*}$ to the short exact sequence

$$
0 \rightarrow \mathcal{I}_{C}(H) \rightarrow \mathcal{O}_{Y}(H) \rightarrow \mathcal{O}_{C}(H) \rightarrow 0
$$

we get

$$
0 \rightarrow \mathcal{H}^{-1}\left(\Psi\left(\sigma^{*} \mathcal{I}_{C}(H)\right)\right) \rightarrow \mathcal{B}_{2} \stackrel{f}{\rightarrow} \Psi\left(\sigma^{*} \mathcal{O}_{C}(H)\right) \rightarrow \mathcal{H}^{0}\left(\Psi\left(\sigma^{*} \mathcal{I}_{C}(H)\right)\right) \rightarrow 0 .
$$

On the other hand, from (3.2.1) we obtain

$$
0 \rightarrow \mathcal{H}^{-1}\left(\Psi\left(\sigma^{*} F\right)\right) \rightarrow \mathcal{H}^{-1}\left(\Psi\left(\sigma^{*} \mathcal{I}_{C}(H)\right)\right) \stackrel{g}{\rightarrow} \mathcal{B}_{-1} \rightarrow \mathcal{H}^{0}\left(\Psi\left(\sigma^{*} F\right)\right) \rightarrow \mathcal{H}^{0}\left(\Psi\left(\sigma^{*} \mathcal{I}_{C}(H)\right)\right) \rightarrow 0 .
$$

Observe that $\mathcal{H}^{-1}\left(\Psi\left(\sigma^{*} \mathcal{I}_{C}(H)\right)\right) \subseteq \mathcal{B}_{2}$ is a non-trivial torsion-free sheaf of rank four. Hence, the map $g$ is either injective or zero.

Step 1: Assume that the associated elliptic quintic $C$ does not intersect $l_{0}$. Since $C \cap l_{0}=\emptyset$, we have $\Psi\left(\sigma^{*} \mathcal{O}_{C}(H)\right)=F_{\widetilde{C}}[1]$, where $F_{\widetilde{C}}$ is a rank two torsion-free bundle supported on the irreducible curve $\widetilde{C}=\pi\left(\sigma^{-1}(C)\right) \subset \mathbb{P}^{2}$. Hence, (3.2.2) becomes

$$
0 \rightarrow \mathcal{H}^{-1}\left(\Psi\left(\sigma^{*} \mathcal{I}_{C}(H)\right)\right) \rightarrow \mathcal{B}_{2} \stackrel{f}{\rightarrow} F_{\widetilde{C}} \rightarrow \mathcal{H}^{0}\left(\Psi\left(\sigma^{*} \mathcal{I}_{C}(H)\right)\right) \rightarrow 0 .
$$

On the one hand, note that $f$ could be surjective, be zero, or have cokernel supported on points. Indeed, by [BMMS12, Lemma 2.13(ii)], the image of $f$ is supported on points, trivial, or a rank two torsion-free subsheaf of $F_{\widetilde{C}}$. As $F_{\widetilde{C}}$ is torsion-free, the first possibility cannot be realized. Thus $f$ has to be as we claimed above.

Now we observe that $g$ in (3.2.3) is injective. Assume, by contradiction, that $g$ is zero. Hence, we have the following exact sequence:

$$
0 \rightarrow \mathcal{B}_{-1} \rightarrow \mathcal{H}^{0}\left(\Psi\left(\sigma^{*} F\right)\right) \rightarrow \mathcal{H}^{0}\left(\Psi\left(\sigma^{*} \mathcal{I}_{C}(H)\right)\right) \rightarrow 0
$$

If $\mathcal{H}^{0}\left(\Psi\left(\sigma^{*} \mathcal{I}_{C}(H)\right)\right)$ is supported at most in dimension zero, then we have

$$
0 \neq \operatorname{Hom}^{2}\left(\mathcal{B}_{1}, \mathcal{B}_{-1}\right) \hookrightarrow \operatorname{Hom}^{2}\left(\mathcal{B}_{1}, \mathcal{H}^{0}(\Psi(F))\right)
$$

Note that we have an exact triangle

$$
\mathcal{H}^{-1}\left(\Psi\left(\sigma^{*} F\right)\right)[1] \rightarrow \Psi\left(\sigma^{*} F\right) \rightarrow \mathcal{H}^{0}\left(\Psi\left(\sigma^{*} F\right)\right),
$$

so this would imply $\operatorname{Hom}^{2}\left(\mathcal{B}_{1}, \Psi\left(\sigma^{*} F\right)\right) \neq 0$. But $F \in \mathbf{T}_{Y}$, so $\Psi\left(\sigma^{*} F\right) \in\left\langle\mathcal{B}_{1}\right\rangle^{\perp}$ and we get a contradiction. If $f$ and $g$ are zero, then $\mathcal{H}^{-1}\left(\Psi\left(\sigma^{*} F\right)\right)=\mathcal{B}_{2}$ and we get a contradiction because $\operatorname{Hom}^{0}\left(\mathcal{B}_{1}, \mathcal{H}^{-1}\left(\Psi\left(\sigma^{*} F\right)\right)\right) \neq 0$. The case when $f$ is surjective and $g$ is trivial can be excluded by a similar argument as we would have $\mathcal{B}_{-1} \cong \mathcal{H}^{0}\left(\Psi\left(\sigma^{*} F\right)\right)$.

Therefore, $g$ is injective and $\Psi\left(\sigma^{*} F\right)$ is a torsion sheaf with class $2\left[\mathcal{B}_{1}\right]-2\left[\mathcal{B}_{0}\right]$.

Step 2: Assume that the associated elliptic quintic $C$ intersects $l_{0}$ transversally in a point. Since $C \cap l_{0}=\{p\}$, we have $\Psi\left(\sigma^{*} \mathcal{O}_{C}(H)\right)=\Psi\left(\mathcal{O}_{C^{\prime} \cup \gamma}(H)\right)$, where by abuse of notation we denote by $C^{\prime}$ the strict transform of $C$ and $\gamma \subset D$ is the line $\sigma^{-1}(p)$. Hence, (3.2.2) becomes

$$
0 \rightarrow \mathcal{H}^{-1}\left(\Psi\left(\sigma^{*} \mathcal{I}_{C}(H)\right)\right) \rightarrow \mathcal{B}_{2} \stackrel{f}{\rightarrow} \Psi\left(\mathcal{O}_{C^{\prime} \cup \gamma}(H)\right) \rightarrow \mathcal{H}^{0}\left(\Psi\left(\sigma^{*} \mathcal{I}_{C}(H)\right)\right) \rightarrow 0 .
$$

To characterize $\Psi\left(\mathcal{O}_{C^{\prime} \cup \gamma}(H)\right)$ better, consider the exact sequence on $\mathbf{C o h}(\widetilde{Y})$

$$
0 \rightarrow \mathcal{I}_{p, C^{\prime}}(H) \rightarrow \mathcal{O}_{C^{\prime} \cup \gamma}(H) \rightarrow \mathcal{O}_{\gamma}(H) \rightarrow 0
$$




\section{ACM BUNDLES ON CUBIC THREEFOLDS}

On the one hand, we need to compute $\Psi\left(\mathcal{O}_{\gamma}(H)\right)$. As $\gamma \subset D$ it makes sense to consider the ideal sheaf $\mathcal{I}_{\gamma, D}$ which is actually equal to $\mathcal{I}_{\sigma^{-1}(p), \sigma^{-1}\left(l_{0}\right)}=\sigma^{*} \mathcal{I}_{p, l_{0}}=\sigma^{*}\left(\mathcal{O}_{l_{0}}(-H)\right)=\mathcal{O}_{D}(-H)$. Now, tensoring the exact sequence

$$
0 \rightarrow \mathcal{O}_{D}(-H) \rightarrow \mathcal{O}_{D} \rightarrow \mathcal{O}_{\gamma} \rightarrow 0
$$

by $D=H-h$, we have

$$
0 \rightarrow \mathcal{O}_{D}(-h) \rightarrow \mathcal{O}_{D}(D) \rightarrow \mathcal{O}_{\gamma}(H-h) \rightarrow 0
$$

By [BMMS12, Exercise 2.11] and applying the functor $\Psi$, it provides the exact triangle

$$
\mathcal{B}_{-1}[1] \rightarrow \mathcal{B}_{0}[1] \rightarrow \Psi\left(\mathcal{O}_{\gamma}(H-h)\right) \text {. }
$$

By construction, we know that $\Psi\left(\mathcal{O}_{\gamma}(H-h)\right)$ is a torsion sheaf in degree -1 and we have the following exact sequence:

$$
0 \rightarrow \mathcal{B}_{-1} \rightarrow \mathcal{B}_{0} \rightarrow \mathcal{H}^{-1}\left(\Psi\left(\mathcal{O}_{\gamma}(H-h)\right)\right) \rightarrow 0 .
$$

By definition $\Psi\left(F \otimes \mathcal{O}_{\widetilde{Y}}(m h)\right)=\pi_{*}\left(F \otimes \mathcal{O}_{\widetilde{Y}}(m h) \otimes \mathcal{E} \otimes \mathcal{O}_{\widetilde{Y}}(h)\right)[1]=\Psi(F) \otimes \mathcal{O}_{\widetilde{Y}}(m h)$. Hence, if we tensor (3.2.6) by $\mathcal{O}_{\widetilde{Y}}(h)$ and we apply $\Psi$ again, we get that $\Psi\left(\mathcal{O}_{\gamma}(H)\right)$ is a torsion sheaf in degree -1 and we get the following exact sequence:

$$
0 \rightarrow \mathcal{B}_{1} \rightarrow \mathcal{B}_{2} \rightarrow \mathcal{H}^{-1}\left(\Psi\left(\mathcal{O}_{\gamma}(H)\right)\right) \rightarrow 0 .
$$

Note that $\operatorname{Hom}\left(\mathcal{B}_{2}, \mathcal{H}^{-1}\left(\Psi\left(\mathcal{O}_{\gamma}(H)\right)\right)\right) \cong \mathbb{C}$.

Using the discussion above and (3.2.5), we get the commutative diagram

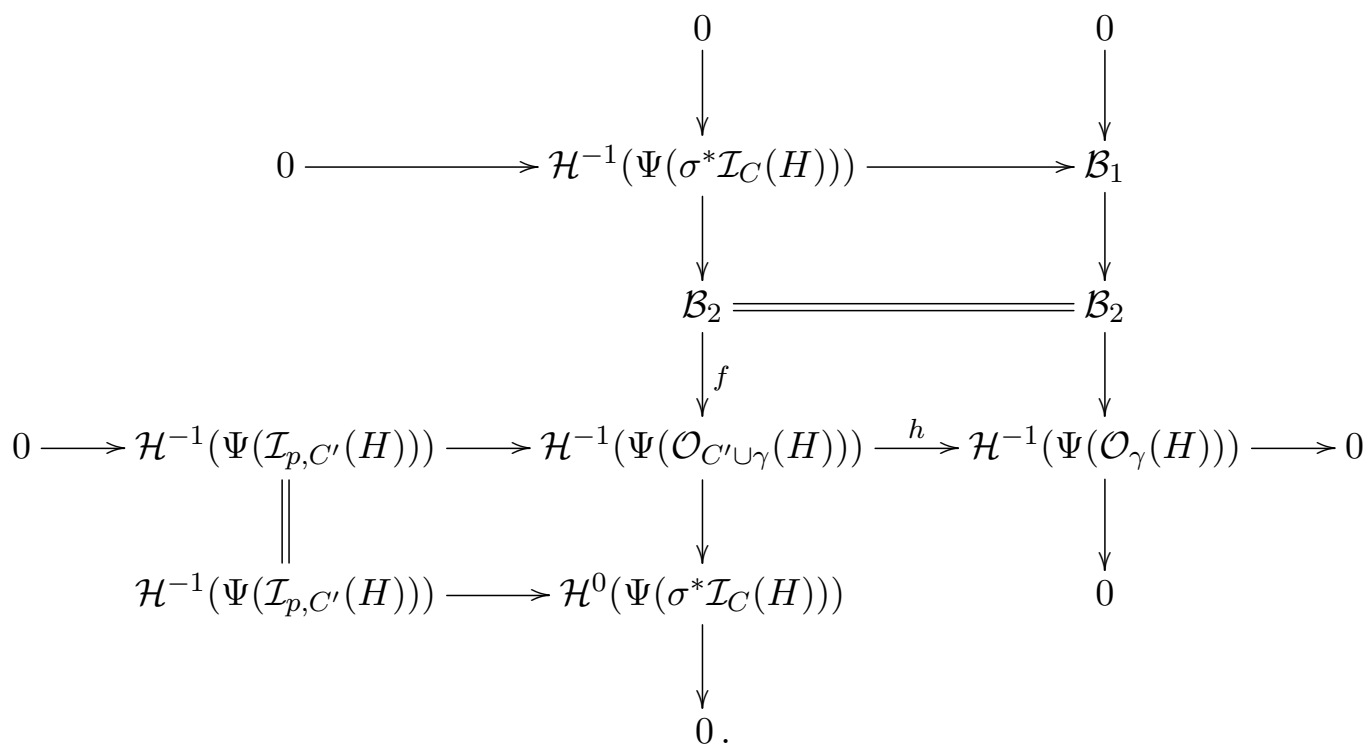

Furthermore, we have $\Psi\left(\mathcal{I}_{p, C^{\prime}}(H)\right) \cong F_{\widetilde{C}^{\prime}}[1]$, where $F_{\widetilde{C^{\prime}}}$ is a rank two torsion-free sheaf supported on $\widetilde{C^{\prime}}=\pi\left(C^{\prime}\right) \subset \mathbb{P}^{2}$ which is irreducible. Indeed, by definition,

$$
\Psi\left(\mathcal{I}_{p, C^{\prime}}(H)\right)=\pi_{*}\left(\mathcal{I}_{p, C^{\prime}}(H) \otimes \mathcal{E} \otimes \mathcal{O}_{\widetilde{Y}}(h)\right)[1] .
$$

Since the fibers of $\pi$ restricted to $C^{\prime}$ are only points or empty, we have

$$
\Psi\left(\mathcal{I}_{p, C^{\prime}}(H)\right)=R^{0} \pi_{*}\left(\mathcal{I}_{p, C^{\prime}}(H) \otimes \mathcal{E} \otimes \mathcal{O}_{\widetilde{Y}}(h)\right)[1],
$$

where $R^{0} \pi_{*}\left(\mathcal{I}_{p, C^{\prime}}(H) \otimes \mathcal{E} \otimes \mathcal{O}_{\widetilde{Y}}(h)\right)$ is a sheaf supported on $\widetilde{C^{\prime}}=\pi\left(C^{\prime}\right)$. Observe that $C^{\prime} \subset \widetilde{Y}$ is a quartic, so the image $\pi\left(C^{\prime}\right)$ is a line, a conic, or a quartic in $\mathbb{P}^{2}$. The first two possibilities are 


\section{Martí Lahoz, Emanuele Macrì and PaOlo Stellari}

not realized and this means that $C^{\prime} \rightarrow \widetilde{C^{\prime}}$ is birational. Hence, by base chance, $R^{0} \pi_{*}\left(\mathcal{I}_{p, C^{\prime}}(H) \otimes\right.$ $\mathcal{E} \otimes \mathcal{O}_{\widetilde{Y}}(h)$ ) has rank on $\widetilde{C^{\prime}}$ equal to the rank of $\mathcal{I}_{p, C^{\prime}}(H) \otimes \mathcal{E} \otimes \mathcal{O}_{\widetilde{Y}}(h)$ on $C^{\prime}$, which is two.

We claim that $g$ in (3.2.3) is injective. Assume, by contradiction, that $g$ is zero. Hence, we have the following exact sequence:

$$
0 \rightarrow \mathcal{B}_{-1} \rightarrow \mathcal{H}^{0}\left(\Psi\left(\sigma^{*} F\right)\right) \rightarrow \mathcal{H}^{0}\left(\Psi\left(\sigma^{*} \mathcal{I}_{C}(H)\right)\right) \rightarrow 0,
$$

and we have two cases depending on the behavior morphism $f^{\prime}:=h \circ f: \mathcal{B}_{2} \rightarrow \mathcal{H}^{-1}\left(\Psi\left(\mathcal{O}_{\gamma}(H)\right)\right)$.

Case (a.1). If the map $f^{\prime}$ is non-zero, then (3.2.8) yields the sequence

$$
0 \rightarrow \mathcal{H}^{-1}\left(\Psi\left(\sigma^{*} \mathcal{I}_{C}(H)\right)\right) \rightarrow \mathcal{B}_{1} \stackrel{f_{1}}{\rightarrow} F_{\widetilde{C}^{\prime}} \rightarrow \mathcal{H}^{0}\left(\Psi\left(\sigma^{*} \mathcal{I}_{C}(H)\right)\right) \rightarrow 0
$$

where $f_{1}$ could be zero, be surjective, or have cokernel supported on points (see Step 1). If coker $f_{1}=\mathcal{H}^{0}\left(\Psi\left(\sigma^{*} \mathcal{I}_{C}(H)\right)\right)$ is supported at most in dimension zero, then we have

$$
0 \neq \operatorname{Hom}^{2}\left(\mathcal{B}_{1}, \mathcal{B}_{-1}\right) \hookrightarrow \operatorname{Hom}^{2}\left(\mathcal{B}_{1}, \mathcal{H}^{0}\left(\Psi\left(\sigma^{*} F\right)\right)\right) .
$$

So we get a contradiction with $\Psi\left(\sigma^{*} F\right) \in\left\langle\mathcal{B}_{1}\right\rangle^{\perp}$. Assume that $f_{1}$ and $g$ are both zero. Then $\mathcal{H}^{-1}\left(\Psi\left(\sigma^{*} F\right)\right)=\mathcal{B}_{1}$ and we get a contradiction as $\operatorname{Hom}^{0}\left(\mathcal{B}_{1}, \mathcal{H}^{-1}\left(\Psi\left(\sigma^{*} F\right)\right)\right) \neq 0$. Hence, $f_{1}$ is surjective and then, as in Step $1, g$ is injective.

Case (b.1). On the other hand, the map $f^{\prime}$ could be zero, in which case $f$ would factor through $\mathcal{B}_{2} \rightarrow \mathcal{H}^{-1}\left(\Psi\left(\mathcal{I}_{p, C^{\prime}}(H)\right)\right)$. In this case, we get a sequence

$$
0 \rightarrow \mathcal{H}^{-1}\left(\Psi\left(\sigma^{*} \mathcal{I}_{C}(H)\right)\right) \rightarrow \mathcal{B}_{2} \stackrel{f_{2}}{\rightarrow} F_{\widetilde{C^{\prime}}} \rightarrow K \rightarrow 0
$$

where $f_{2}$ could be zero, be surjective, or have cokernel supported on points (see again Step 1). Moreover, we have

$$
0 \rightarrow K \rightarrow \mathcal{H}^{0}\left(\Psi\left(\sigma^{*} \mathcal{I}_{C}(H)\right)\right) \rightarrow \mathcal{H}^{-1}\left(\Psi\left(\mathcal{O}_{\gamma}(H)\right)\right) \rightarrow 0
$$

If $K$ is supported at most in dimension zero, then

$$
\operatorname{Ext}^{1}\left(\mathcal{B}_{1}, \mathcal{H}^{0}\left(\Psi\left(\sigma^{*} \mathcal{I}_{C}(H)\right)\right)\right)=\operatorname{Ext}^{1}\left(\mathcal{B}_{1}, \mathcal{H}^{-1}\left(\Psi\left(\mathcal{O}_{\gamma}(H)\right)\right)\right)=0,
$$

by (3.2.9) and (3.2.7). So, again we have

$$
0 \neq \operatorname{Hom}^{2}\left(\mathcal{B}_{1}, \mathcal{B}_{-1}\right) \hookrightarrow \operatorname{Hom}^{2}\left(\mathcal{B}_{1}, \mathcal{H}^{0}\left(\Psi\left(\sigma^{*} F\right)\right)\right),
$$

contradicting the fact that $\Psi\left(\sigma^{*} F\right) \in\left\langle\mathcal{B}_{1}\right\rangle^{\perp}$. If $f_{2}$ and $g$ are zero, then $\mathcal{H}^{-1}\left(\Psi\left(\sigma^{*} F\right)\right)=\mathcal{B}_{2}$ and we get a contradiction because $\operatorname{Hom}^{0}\left(\mathcal{B}_{1}, \mathcal{H}^{-1}\left(\Psi\left(\sigma^{*} F\right)\right)\right) \neq 0$. As in the previous step, $f$ cannot be surjective whenever $g$ is trivial.

Therefore, $g$ is injective and $\Psi\left(\sigma^{*} F\right)$ is a torsion sheaf with class $2\left[\mathcal{B}_{1}\right]-2\left[\mathcal{B}_{0}\right]$.

Step 3: Assume that the associated elliptic quintic $C$ intersects $l_{0}$ with multiplicity $m$ in a point. Since $C \cap l_{0}=\{p\}$ with multiplicity $m>1$, with the notation of Step 2, we have $\Psi\left(\sigma^{*} \mathcal{O}_{C}(H)\right)=$ $\Psi\left(\mathcal{O}_{C^{\prime} \cup m \gamma}(H)\right)$. Note that we have the following exact sequence on $\mathbf{C o h}(\widetilde{Y})$ :

$$
0 \rightarrow \mathcal{I}_{m p, C^{\prime}}(H) \rightarrow \mathcal{O}_{C^{\prime} \cup m \gamma}(H) \rightarrow \mathcal{O}_{m \gamma}(H) \rightarrow 0 .
$$

Moreover, we have the exact sequence

$$
0 \rightarrow \mathcal{O}_{(m-1) \gamma}(H) \rightarrow \mathcal{O}_{m \gamma}(H) \rightarrow \mathcal{O}_{\gamma}(H) \rightarrow 0,
$$

so $\Psi\left(\mathcal{O}_{m \gamma}(H)\right)$ is a successive extension of $\Psi\left(\mathcal{O}_{\gamma}(H)\right)$. 


\section{ACM BUNDLES ON CUBIC THREEFOLDS}

Then, we can also distinguish between two cases, depending on whether the morphism

$$
\mathcal{B}_{2} \rightarrow \mathcal{H}^{-1}\left(\Psi\left(\mathcal{O}_{m \gamma}(H)\right)\right)
$$

arising from (3.2.10) and (3.2.2) is non-zero (case (a.2)), or $f$ factors through the morphism $\mathcal{B}_{2} \rightarrow \mathcal{H}^{-1}\left(\Psi\left(\mathcal{I}_{p, C^{\prime}}(H)\right)\right)$ as in (3.2.2) (case (b.2)).

If we are in case (b.2), exactly the same arguments as in case (b.1) show that $\Psi\left(\sigma^{*} F\right)$ is a torsion sheaf with class $2\left[\mathcal{B}_{1}\right]-2\left[\mathcal{B}_{0}\right]$. So we can suppose that we are in case (a.2) and we have the following diagram:

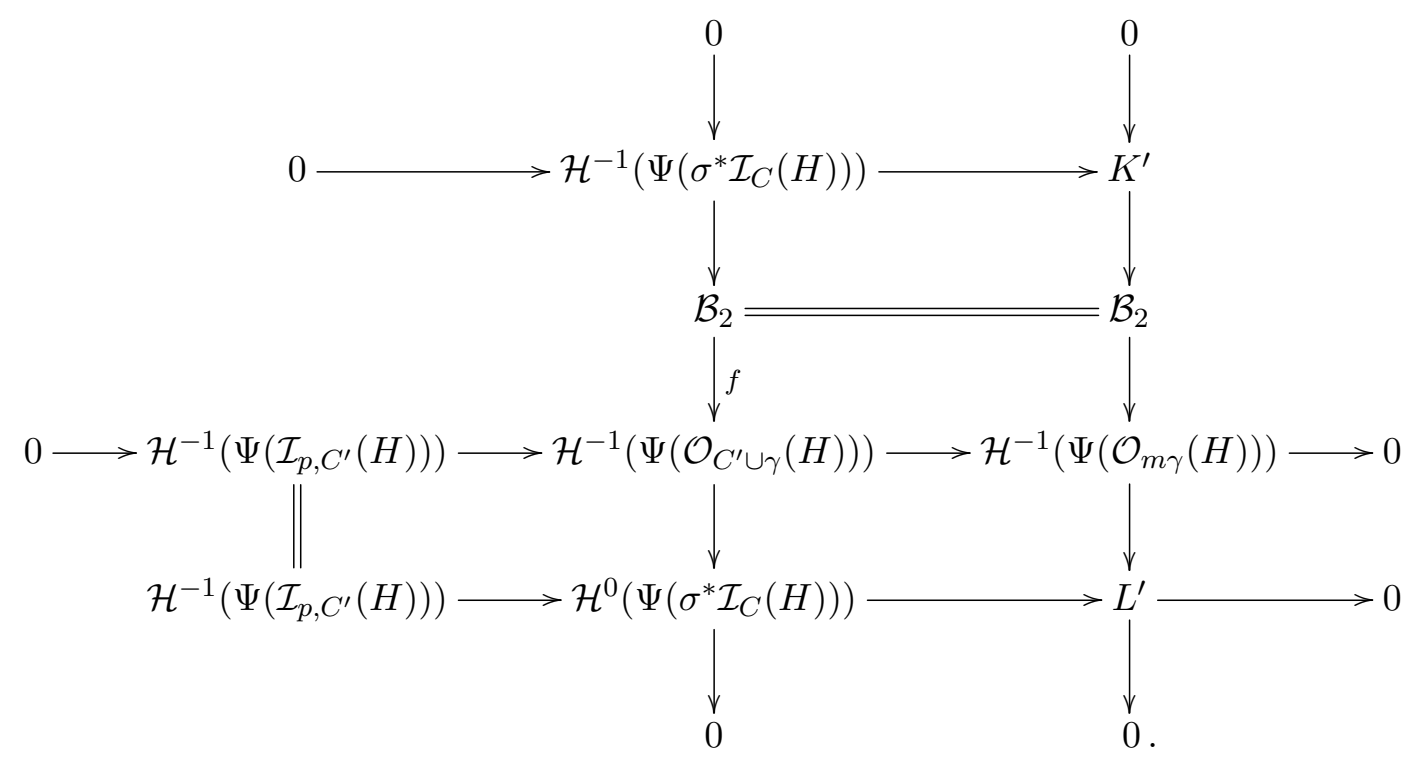

By the horseshoe lemma we have the following exact sequence:

$$
0 \rightarrow \mathcal{B}_{1}^{\oplus m} \rightarrow \mathcal{B}_{2}^{\oplus m} \rightarrow \mathcal{H}^{-1}\left(\Psi\left(\mathcal{O}_{m \gamma}(H)\right)\right) \rightarrow 0 .
$$

So, we have $K^{\prime} \cong \mathcal{B}_{1}$ and $L^{\prime} \cong \mathcal{H}^{-1}\left(\Psi\left(\mathcal{O}_{(m-1) \gamma}(H)\right)\right)$ and we get the following exact sequence:

$$
0 \rightarrow \mathcal{H}^{-1}\left(\Psi\left(\sigma^{*} \mathcal{I}_{C}(H)\right)\right) \rightarrow \mathcal{B}_{1} \stackrel{f_{3}}{\rightarrow} F_{\widetilde{C^{\prime}}} \rightarrow \mathcal{H}^{0}\left(\Psi\left(\sigma^{*} \mathcal{I}_{C}(H)\right)\right) \rightarrow \mathcal{H}^{-1}\left(\Psi\left(\mathcal{O}_{(m-1) \gamma}(H)\right)\right) \rightarrow 0,
$$

where $f_{3}$ could be zero, be surjective, or have cokernel supported on points (see the argument in Step 1).

We claim that $g$ in (3.2.3) is injective. Running the same machinery as in the previous steps, we assume, by contradiction, that $g$ is zero. Hence, we have the following exact sequence:

$$
0 \rightarrow \mathcal{B}_{-1} \rightarrow \mathcal{H}^{0}\left(\Psi\left(\sigma^{*} F\right)\right) \rightarrow \mathcal{H}^{0}\left(\Psi\left(\sigma^{*} \mathcal{I}_{C}(H)\right)\right) \rightarrow 0
$$

If coker $f_{3}$ is supported at most in dimension zero,

$$
\operatorname{Ext}^{1}\left(\mathcal{B}_{1}, \mathcal{H}^{0}\left(\Psi\left(\sigma^{*} \mathcal{I}_{C}(H)\right)\right)=\operatorname{Ext}^{1}\left(\mathcal{B}_{1}, \mathcal{H}^{0}\left(\Psi\left(\mathcal{O}_{m \gamma}(H)\right)\right)\right)=0\right.
$$

So, we have $0 \neq \operatorname{Hom}^{2}\left(\mathcal{B}_{1}, \mathcal{B}_{-1}\right) \hookrightarrow \operatorname{Hom}^{2}\left(\mathcal{B}_{1}, \mathcal{H}^{0}\left(\Psi\left(\sigma^{*} F\right)\right)\right)$, contradicting $\Psi\left(\sigma^{*} F\right) \in\left\langle\mathcal{B}_{1}\right\rangle^{\perp}$. Thus, it remains to deal with the case when $f_{3}$ and $g$ are zero. But then $\mathcal{H}^{-1}\left(\Psi\left(\sigma^{*} F\right)\right)=\mathcal{B}_{1}$ and we get a contradiction because $\operatorname{Hom}^{0}\left(\mathcal{B}_{1}, \mathcal{H}^{-1}\left(\Psi\left(\sigma^{*} F\right)\right)\right) \neq 0$.

If $f$ factors through $\mathcal{B}_{2} \rightarrow \mathcal{H}^{-1}\left(\Psi\left(\mathcal{I}_{p, C^{\prime}}(H)\right)\right)$ and $K$ is supported at most in dimension zero, then

$$
\operatorname{Ext}^{1}\left(\mathcal{B}_{1}, \mathcal{H}^{0}\left(\Psi\left(\sigma^{*} \mathcal{I}_{C}(H)\right)\right)\right)=\operatorname{Ext}^{1}\left(\mathcal{B}_{1}, \mathcal{H}^{-1}\left(\Psi\left(\mathcal{O}_{\gamma}(H)\right)\right)\right)=0
$$




\section{Martí Lahoz, Emanuele Macrì and PaOlo Stellari}

by (3.2.9) and (3.2.7). So, again we have $0 \neq \operatorname{Hom}^{2}\left(\mathcal{B}_{1}, \mathcal{B}_{-1}\right) \hookrightarrow \operatorname{Hom}^{2}\left(\mathcal{B}_{1}, \mathcal{H}^{0}\left(\Psi\left(\sigma^{*} F\right)\right)\right)$ and we get a contradiction with $\Psi\left(\sigma^{*} F\right) \in\left\langle\mathcal{B}_{1}\right\rangle^{\perp}$. As in the previous steps, if $f_{2}$ and $g$ are zero, then $\mathcal{H}^{-1}\left(\Psi\left(\sigma^{*} F\right)\right)=\mathcal{B}_{2}$ and we get a contradiction because $\operatorname{Hom}^{0}\left(\mathcal{B}_{1}, \mathcal{H}^{-1}\left(\Psi\left(\sigma^{*} F\right)\right)\right) \neq 0$. Therefore, $\Psi\left(\sigma^{*} F\right)$ is a torsion sheaf with class $2\left[\mathcal{B}_{1}\right]-2\left[\mathcal{B}_{0}\right]$.

Step 4: Assume that the associated elliptic quintic $C$ intersects $l_{0}$ in $s$ distinct points (possibly with multiplicity). Since $C \cap l_{0}=\left\{p_{1}, \ldots, p_{s}\right\}$, with the notation of Steps 2 and 3 , we have $\Psi\left(\sigma^{*} \mathcal{O}_{C}(H)\right)=\Psi\left(\mathcal{O}_{C^{\prime} \cup m_{1} \gamma_{1} \cup \ldots \cup m_{s} \gamma_{s}}(H)\right)$. Note that we have the following exact sequence in $\operatorname{Coh}(\widetilde{Y})$ :

$$
0 \rightarrow \mathcal{I}_{m_{1} p_{1} \cup \ldots \cup m_{s} p_{s}, C^{\prime}}(H) \rightarrow \mathcal{O}_{C^{\prime} \cup m_{1} \gamma_{1} \cup \ldots \cup m_{s} \gamma_{s}}(H) \rightarrow \bigoplus_{i=1}^{s} \mathcal{O}_{m_{i} \gamma_{i}}(H) \rightarrow 0,
$$

since the lines $\gamma_{i}$ are disjoint. Then, we can reduce to the previous steps.

From Step 1-4, we get that if $F$ is a stable Ulrich bundle of rank two, then $\Psi\left(\sigma^{*} F\right)$ is a torsion sheaf with class $2\left[\mathcal{B}_{1}\right]-2\left[\mathcal{B}_{0}\right]$.

Step 5: We can now show that $\Psi\left(\sigma^{*} F\right)$ is stable. Suppose it is not, and let $E \hookrightarrow \Psi\left(\sigma^{*} F\right)$ be a destabilizing $\mathcal{B}_{0}$-module. Then, $\operatorname{ch}(E)=(0,2, z)$ for some $z>-2$. Since

$$
\operatorname{Hom}\left(\mathcal{B}_{1}, E\right) \hookrightarrow \operatorname{Hom}\left(\mathcal{B}_{1}, \Psi\left(\sigma^{*} F\right)\right)=0,
$$

we have $\chi\left(\mathcal{B}_{1}, E\right) \leqslant 0$. By (2.2.3), we have $z=-2$ and $E \in\left\langle\mathcal{B}_{1}\right\rangle^{\perp}$. By [BMMS12, Theorem 4.1], we get $E \cong \Xi_{3}\left(\mathcal{I}_{l}\right)$ for some line $l \in F(Y) \backslash\left\{l_{0}\right\}$ and $\mathcal{I}_{l} \hookrightarrow F$ contradicts the stability of $F$.

Denote by $\mathfrak{M}_{Y}^{\text {inst }}$ the moduli space of semistable instanton sheaves. By [Dru00, Theorem 4.8], $\mathfrak{M}_{Y}^{\text {inst }}$ is isomorphic to the blow-up $f: \mathfrak{M}_{Y}^{\text {inst }} \rightarrow J(Y)$ of the intermediate Jacobian $J(Y)$ of $Y$ along (a translate of) $-F(Y)$. Here $F(Y)$ is the Fano surface of lines in $Y$. Recall that the AbelJacobi map establishes an isomorphism $\operatorname{Alb}(F(Y)) \stackrel{\sim}{\rightarrow} J(Y)$. Moreover, the Albanese morphism provides an embedding $F(Y) \hookrightarrow \operatorname{Alb}(F(Y))$ defined after picking a special point, in our case $l_{0}$ (see [CG72]). Recall that the closure of the locus of stable non-locally-free instanton sheaves (case (2) in Remark 3.8) forms the exceptional divisor of $f$. Stable non-locally-free instanton sheaves are associated with a smooth conic inside $Y$ via the Serre construction and they are sent to the residual line of the conic.

We denote by $\overline{F(Y)}$ the strict transform of $F(Y)$ under $f$. Since we have chosen $l_{0}$ general (that is, such that for any other line $l$ meeting $l_{0}$, the plane containing them intersects the cubic in three distinct lines), $F(Y) \cap(-F(Y))$ is the Abel-Prym curve $C_{l_{0}} \subset J(Y)$ consisting of all lines inside $Y$ that intersect $l_{0}$ (see, for example, [LN13, Section 5$]$ ). Note that $\overline{F(Y)}$ parametrizes properly semistable instanton sheaves that fall under case (3) in Remark 3.8 and are extensions of $\mathcal{I}_{l_{0}}$ and $\mathcal{I}_{l}$, for $l$ a line in $Y$ (possibly equal to $l_{0}$ ). Indeed, semistable instanton sheaves under case (3) have second Chern class $c_{2}(E)=l+l_{0}$.

Therefore, $\overline{F(Y)}$ intersects the divisor contracted by $f$, in the locus where $E$ is semistable, and it is the extension of $\mathcal{I}_{l_{0}}$ and $\mathcal{I}_{l}$ with $l \cap l_{0} \neq \emptyset$. From the point of view of the conics, this corresponds to the case when the conic over $l$ degenerates to $l_{0} \cup l^{\prime}$, where $l, l^{\prime}, l_{0}$ are coplanar and in general position.

Theorem 3.10. The moduli space $\mathfrak{M}_{2}$ is the blow-up of $\mathfrak{M}_{Y}^{\text {inst }}$ along $\overline{F(Y)}$. 


\section{ACM BUNDLES ON CUBIC THREEFOLDS}

Proof. Let $E$ be a semistable instanton sheaf. We claim that if $\Xi_{3}(E) \in \mathbf{C o h}\left(\mathbb{P}^{2}, \mathcal{B}_{0}\right)$, then $\Xi_{3}(E) \in \mathfrak{M}_{2}$ (that is, it is semistable) and, by Lemma 3.1, $\Xi_{3}(E) \in \mathfrak{M}^{\sigma_{m}}\left(\mathbb{P}^{2}, \mathcal{B}_{0} ; w\right)$ for all $m>\frac{1}{4}$. Combining Remark 3.8 and [BMMS12, Example 2.4, Example 2.11, Step 5 in Proposition 3.3], we can distinguish three cases where $\Xi_{3}(E) \in \mathbf{C o h}\left(\mathbb{P}^{2}, \mathcal{B}_{0}\right)$.

One possibility is that $E$ is a stable instanton bundle. In this case, $\Xi_{3}(E) \in \mathfrak{M}_{2}$ follows from Lemma 3.9.

Another possibility is that $E$ is a stable instanton sheaf which is not locally free. In that case $E$ can be associated with a smooth conic via the Serre construction. If the conic does not intersect the line of projection, then $\Xi_{3}(E) \in \mathfrak{M}_{2}$ follows from Example 2.10. If the conic intersects the line of projection in one point or two points (even tangentially), then the same computations as in Steps 2, 3, and 4 of the proof of Lemma 3.9 show again that $\Xi_{3}(E) \in \mathbf{C o h}\left(\mathbb{P}^{2}, \mathcal{B}_{0}\right)$. By Step 5 of the proof of Lemma $3.9, \Xi_{3}(E)$ is stable, so in $\mathfrak{M}_{2}$.

Finally, the last possibility is that $E$ is a properly semistable sheaf and the two JH-factors are $\mathcal{I}_{l}$ and $\mathcal{I}_{l^{\prime}}$, where we may have $l=l^{\prime}$, but in any case $l, l^{\prime} \neq l_{0}$. Then $\Xi_{3}(E) \in \mathfrak{M}_{2}$ follows from a direct computation based on the fact that $\Xi_{3}\left(\mathcal{I}_{l}\right)$ and $\Xi_{3}\left(\mathcal{I}_{l^{\prime}}\right)$ are in $\mathfrak{M}_{1}^{s}$ (see Lemma 2.14).

Hence, by [BMMS12, Exercise 2.11], the only cases in which $\Xi_{3}(E) \notin \mathbf{C o h}\left(\mathbb{P}^{2}, \mathcal{B}_{0}\right)$ appear when $E$ is a properly semistable sheaf and $\mathcal{I}_{l_{0}}$ is a JH-factor. Indeed, this is the only case where $\Xi_{3}(E) \notin \mathfrak{M}_{2}$ and we need to push our analysis a bit further.

When $\mathcal{I}_{l_{0}}$ and $\mathcal{I}_{l}$ with $l \neq l_{0}$ are the JH-factors of $E$, $\operatorname{hom}\left(\Xi_{3}(E), \mathcal{B}_{1}\right)=1$. Hence, the HN-filtration of $\Xi_{3}(E)$ for $m>m_{0}=\frac{\sqrt{5}}{8}$ is

$$
\mathcal{B}_{0}[1] \subset C[1] \subset \Xi_{3}(E),
$$

where $0 \rightarrow C[1] \rightarrow \Xi_{3}(E) \rightarrow \mathcal{B}_{1} \rightarrow 0$ and $0 \rightarrow \mathcal{B}_{0}[1] \rightarrow C[1] \rightarrow \Xi_{3}\left(\mathcal{I}_{l}\right) \rightarrow 0$ are exact sequences in the abelian category $\mathbf{A}$ which is the heart of the bounded $t$-structure in the stability condition in Lemma 2.7. If the two JH-factors of $E$ are both isomorphic to $\mathcal{I}_{l_{0}}$, then $\operatorname{hom}\left(\Xi_{3}(E), \mathcal{B}_{1}\right)=2$. Hence, the HN-filtration of $\Xi_{3}(E)$ for $m>m_{0}$ is

$$
\mathcal{B}_{0}[1] \subset \mathcal{B}_{0}^{\oplus 2}[1] \subset C[1] \subset \Xi_{3}(E),
$$

where $0 \rightarrow \mathcal{B}_{0}^{\oplus 2}[1] \rightarrow C[1] \rightarrow \mathcal{B}_{1} \rightarrow 0$ and $0 \rightarrow C[1] \rightarrow \Xi_{3}(E) \rightarrow \mathcal{B}_{1} \rightarrow 0$ are exact sequences in the abelian category $\mathbf{A}$.

In both cases, this means that $\Xi_{3}(E)$ is $\sigma_{m_{0}}$-semistable. As a consequence, up to choosing $\varepsilon$ small enough, $\Xi_{3}(E)$ is $\sigma_{m}$-semistable for all $m \in\left(m_{0}-\varepsilon, m_{0}\right)$. Indeed, if not, by [Bri08, Proposition 9.3], the HN-factors of $\Xi_{3}(E)$ in the stability condition $\sigma_{m}$, for $m \in\left(m_{0}-\varepsilon, m_{0}\right)$, would survive in the stability condition $\sigma_{m_{0}}$. This would contradict the $\sigma_{m_{0}}$-semistability of $\Xi_{3}(E)$.

Since the quotient $\Xi_{3}(E) \rightarrow \mathcal{B}_{1} \sigma_{m}$-destabilizes $\Xi_{3}(E)$, for $m>m_{0}$, we have

$$
\begin{array}{ll}
\Xi_{3}(E) \notin \mathfrak{M}^{\sigma_{m}}\left(\mathbb{P}^{2}, \mathcal{B}_{0} ; w\right) & \text { for } m>m_{0}:=\frac{\sqrt{5}}{8}, \\
\Xi_{3}(E) \in \mathfrak{M}^{\sigma_{m}}\left(\mathbb{P}^{2}, \mathcal{B}_{0} ; w\right) & \text { for } m \in\left(m_{0}-\varepsilon, m_{0}\right] \text { and } \varepsilon>0 \text { small enough, }
\end{array}
$$

where $w:=2\left[\mathcal{B}_{1}\right]-2\left[\mathcal{B}_{0}\right]$.

We claim that $\mathfrak{M}_{Y}^{\text {inst }}=\mathfrak{M}^{\sigma_{m}}\left(\mathbb{P}^{2}, \mathcal{B}_{0} ; w\right)$ for all $m \in\left(m_{0}-\varepsilon, m_{0}\right]$ and $\varepsilon>0$ sufficiently small. More precisely, we need to show that, for $m \in\left(m_{0}-\varepsilon, m_{0}\right)$, the objects $\Xi_{3}(E)$ are the only $\sigma_{m}$-semistable objects in $\mathbf{A}$ with class $w$, when $E$ is a semistable instanton sheaf. First observe that, if $G \in \mathbf{A}$ is a $\sigma_{m}$-semistable object, for some $m \in\left(m_{0}-\varepsilon, m_{0}\right)$ and with class $w$, then 


\section{Martí Lahoz, Emanuele Macrì and PaOlo Stellari}

$G \in\left\langle\mathcal{B}_{1}\right\rangle^{\perp}$. Indeed, if $\operatorname{hom}\left(\mathcal{B}_{1}, G\right) \neq 0$, then the image $T:=\operatorname{Im}_{\mathbf{A}}\left(\mathcal{B}_{1} \rightarrow G\right)$ destabilizes $G$, for $m \in\left(m_{0}-\varepsilon, m_{0}\right)$ and $\varepsilon>0$ small enough, since $\operatorname{Re}\left(Z_{m_{0}}(T)\right)>0$.

By [Bri08, Proposition 9.3], up to replacing $\varepsilon$, we can assume that all such objects $G$ are $\sigma_{m_{0}}{ }^{-}$ semistable. By Lemma 3.2, we have two possibilities: either $G$ is $\sigma_{m}$-semistable for all $m \geqslant m_{0}$, or $G$ is properly $\sigma_{m_{0}}$-semistable and destabilizes for all $m>m_{0}$. In the first case, $G$ is a (semi)stable element of $\mathfrak{N}_{2}$ by Lemma 3.3 and the discussion above. Thus, by the proof of Proposition 2.22, $\Xi_{3}^{-1}(G)$ is either a balanced ACM bundle of rank two (that is, an instanton bundle) or as in case (2) of Remark 3.8.

If $G$ destabilizes for all $m>m_{0}$, then $G$ needs to be in cases (a) and (b) of Lemma 3.5. Since $G \in\left\langle\mathcal{B}_{1}\right\rangle^{\perp}$, Lemma 3.5 tell us that $G \cong \Xi_{3}(E)$, where $E$ is a properly semistable sheaf with $\mathcal{I}_{l_{0}}$ as a $\mathrm{JH}$-factor.

Having proved this, we are ready to show that $\mathfrak{M}_{2}$ is the blow-up of $\mathfrak{M}_{Y}^{\text {inst }}$ along $\overline{F(Y)}$. In view of Corollary 3.4, one has to study the objects $F \in \mathfrak{M}^{\sigma_{m}}\left(\mathbb{P}^{2}, \mathcal{B}_{0} ; w\right)=\mathfrak{M}_{2}$ for all $m>m_{0}$ and $w=2\left[\mathcal{B}_{1}\right]-2\left[\mathcal{B}_{0}\right]$, which become $\sigma_{m_{0}}$-semistable with JH-factors as in cases (c.iii) and (c.iv) of Lemma 3.1. Indeed, by Lemma 3.1, these are the only objects that could be contracted. By Remark 3.6, the ones falling in case (a.i) of Lemma 3.5 get contracted to the S-equivalence classes of the instanton sheaves which are extensions of $\mathcal{I}_{l_{0}}$ and $\mathcal{I}_{l}\left(l \neq l_{0}\right)$. For the same reason, the ones in case (b.i) of Lemma 3.5 are contracted to the S-equivalence class of the instanton sheaves which are extensions of $\mathcal{I}_{l_{0}}$ with itself. Moreover, again by Lemma 3.5, each contracted fiber is $\mathbb{P}^{2}$ and the birational map $\mathfrak{M}_{2} \rightarrow \mathfrak{M}_{Y}^{\text {inst }}$ is a well-defined morphism. $\overline{F(Y)}$.

Applying [Luo93, Theorem 2], we conclude that $\mathfrak{M}_{2}$ is isomorphic to the blow-up of $\mathfrak{M}_{Y}^{\text {inst }}$ at

As a corollary of the previous proof we get the following result, which is of interest in itself.

Corollary 3.11. Let $w=2\left[\mathcal{B}_{1}\right]-2\left[\mathcal{B}_{0}\right]$ and $m_{0}=\frac{\sqrt{5}}{8}$. Then $\mathfrak{M}_{Y}^{\text {inst }}=\mathfrak{M}^{\sigma_{m}}\left(\mathbb{P}^{2}, \mathcal{B}_{0} ; w\right)$, for all $m \in\left(m_{0}-\varepsilon, m_{0}\right]$ and $\varepsilon>0$ sufficiently small.

\section{ACKNOWLEDGEMENTS}

It is a pleasure to thank Nick Addington, Asher Auel, Marcello Bernardara, Robin Hartshorne, Daniel Huybrechts, Nathan Ilten, Sukhendu Mehrotra, Nicolas Perrin, Antonio Rapagnetta, Pawel Sosna, and Olivier Wittenberg for very useful conversations and comments. We are also very grateful to the referee for pointing out several inaccuracies and a mistake in the statement of an early version of Theorem A. Parts of this paper were written while the three authors were visiting the University of Utah, the University of Bonn, the Ohio State University, and the University of Barcelona. The warm hospitality from these institutions is gratefully acknowledged.

\section{REFERENCES}

AGV08 D. Abramovich, T. Graber, and A. Vistoli, Gromov-Witten theory of Deligne-Mumford stacks, Amer. J. Math. 130 (2008), 1337-1398. http://dx.doi.org/10.1353/ajm.0.0017

Arr07 E. Arrondo, A Home-Made Hartshorne-Serre Correspondence, Rev. Mat. Complut. 20 (2007), 423-443. http://dx.doi.org/10.5209/rev_REMA.2007.v20.n2.16502

AG99 E. Arrondo and B. Graña, Vector bundles on $G(1,4)$ without intermediate cohomology, J. Algebra 214 (1999), 128-142. http://dx.doi.org/10.1006/jabr.1998.7700 


\section{ACM BUNDLES ON CUBIC THREEFOLDS}

AM09 E. Arrondo and C. Madonna, Curves and vector bundles on quartic threefolds, J. Korean Math. Soc. 46 (2009), 589-607. http://dx.doi.org/10.4134/JKMS. 2009.46.3.589

BM11 A. Bayer and E. Macrì, The space of stability conditions on the local projective plane, Duke Math. J. 160 (2011), 263-322. http://dx.doi.org/10.1215/00127094-1444249

BM13 L MMP for moduli of sheaves on K3s via wall-crossing: nef and movable cones, Lagrangian fibrations, Invent. Math. 198 (2014), 505-590.

BM14 - Projectivity and birational geometry of Bridgeland moduli spaces, J. Amer. Math. Soc. 27 (2014), 707-752. http://dx.doi.org/10.1090/S0894-0347-2014-00790-6

Beau77 A. Beauville, Variétés de Prym et jacobiennes intermédiaires, Ann. Sci. Éc. Norm. Supér. 10 (1977), 309-391.

Beau81_, Sous-variétés spéciales des variétés de Prym, Compos. Math. 45 (1981), 357-383.

Beau02 Vector bundles on the cubic threefold, Symposium in Honor of C.H. Clemens (Salt Lake City, 2000), 71-86, Contemp. Math., vol. 312, Amer. Math. Soc., Providence, 2002.

BB13 M. Bernardara and M. Bolognesi, Derived categories and rationality of conic bundles, Compos. Math. 149 (2013), 1789-1817. http://dx.doi.org/10.1112/S0010437X13007392

BMMS12 M. Bernardara, E. Macrì, S. Mehrotra, and P. Stellari, A categorical invariant for cubic threefolds, Adv. Math. 229 (2012), 770-803. http://dx.doi.org/10.1016/j.aim.2011.10.007

BBR08 I. Biswas, J. Biswas, and G.V. Ravindra, On some moduli spaces of stable vector bundles on cubic and quartic threefolds, J. Pure Appl. Algebra 212 (2008), 2298-2306. http://dx.doi. org $/ 10.1016 / j \cdot j$ paa.2008.03.029

BF09 M.C. Brambilla and D. Faenzi, Moduli spaces of arithmetically Cohen-Macaulay bundles on Fano manifolds of the principal series, Boll. Unione Mat. Ital. 2 (2009), 71-91.

Bri07 T. Bridgeland, Stability conditions on triangulated categories, Ann. Math. 166 (2007), 317-346. http://dx.doi.org/10.4007/annals.2007.166.317

Bri08_ Stability conditions on K3 surfaces, Duke Math. J. 141 (2008), 241-291. http://dx. doi.org/10.1215/S0012-7094-08-14122-5

Cad07 C. Cadman, Using stacks to impose tangency conditions on curves, Amer. J. Math. 129 (2007), 405-427. http://dx.doi.org/10.1353/ajm.2007.0007

Căl00 A. Căldăraru, Derived categories of twisted sheaves on Calabi-Yau manifolds, PhD-Thesis, Cornell University (2000), available at http://www.math.wisc.edu/ andreic/publications/ ThesisSingleSpaced.pdf

CF09 L. Chiantini and D. Faenzi, Rank 2 arithmetically Cohen-Macaulay bundles on a general quintic surface, Math. Nachr. 282 (2009), 1691-1708. http://dx.doi.org/10.1002/mana.200610825

CG72 C.H. Clemens and P. Griffiths, The intermediate Jacobian of the cubic threefold, Ann. Math. 95 (1972), 281-356. http://dx.doi.org/10.2307/1970801

CH11 M. Casanellas and R. Hartshorne, ACM bundles on cubic surfaces, J. Eur. Math. Soc. 13 (2011), 709-731. http://dx.doi.org/10.4171/JEMS/265

CHGS12 M. Casanellas, R. Hartshorne, F. Gleiss, and F.-O. Schreyer, Stable Ulrich bundles, Int. J. Math. 23 (2012) 1250083-1250133. http://dx.doi.org/10.1142/S0129167X12500838

CM05 L. Chiantini and C. K. Madonna, ACM bundles on general hypersurfaces in $\mathbb{P}^{5}$ of low degree, Collect. Math. 56 (2005), 85-96.

Dru00 S. Druel, Espace des modules des faisceaux de rang 2 semi-stables de classes de Chern $c_{1}=0$, $c_{2}=2$ et $c_{3}=0$ sur la cubique de $\mathbb{P}^{4}$, Int Math. Res. Not. 2000, 985-1004.

Eis80 D. Eisenbud, Homological algebra on a complete intersection, with an application to group representations, Trans. Amer. Math. Soc. 260 (1980), 35-64. http://dx.doi.org/10.1090/ S0002-9947-1980-0570778-7

HUB91 J. Herzog, B. Ulrich, and J. Backelin, Linear maximal Cohen-Macaulay modules over strict complete intersections, J. Pure Appl. Algebra 71 (1991), 187-202. http://dx.doi.org/10. 1016/0022-4049(91) 90147-T 


\section{Martí Lahoz, Emanuele Macrì and PaOlo Stellari}

Huy06 D. Huybrechts, Fourier-Mukai transforms in algebraic geometry, Oxford Math. Monogr., Oxford Univ. Press, New York, 2006. http://dx.doi.org/10.1093/acprof: oso/9780199296866.001.0001

HL10 D. Huybrechts and M. Lehn, The geometry of moduli spaces of sheaves, second edition, Cambridge Math. Lib., Cambridge Univ. Press, Cambridge, 2010. http://dx.doi.org/10.1017/ CB09780511711985

Ili99 A. Iliev, Minimal sections of conic bundles, Boll. Unione Mat. Ital. 8 (1999), 401-428.

IM00 A. Iliev and D. Markushevich, The Abel-Jacobi map for a cubic threefold and periods of Fano threefolds of degree 14, Doc. Math. 5 (2000), 23-47.

KLS06 D. Kaledin, M. Lehn, and C. Sorger, Singular symplectic moduli spaces, Invent. Math. 164 (2006), 591-614. http://dx.doi.org/10.1007/s00222-005-0484-6

KM09 A. Kuznetsov and D. Markushevich, Symplectic structures on moduli spaces of sheaves via the Atiyah class, J. Geom. Phys. 59 (2009), 843-860. http://dx.doi.org/10.1016/j.geomphys . 2009.03 .008

KS08 M. Kontsevich and Y. Soibelman, Stability structures, motivic Donaldson-Thomas invariants and cluster transformations, arXiv:0811.2435.

Kuz04 A. Kuznetsov, Derived category of a cubic threefold and the variety $V_{14}$, Proc. Steklov Inst. Math. 246 (2004), 171-194.

Kuz07 A. Kuznetsov, Homological projective duality, Publ. Math. Inst. Hautes Études Sci. 105 (2007), 157-220. http://dx.doi.org/10.1007/s10240-007-0006-8

Kuz08 A. Kuznetsov, Derived categories of quadric fibrations and intersections of quadrics, Adv. Math. 218 (2008), 1340-1369. http://dx.doi.org/10.1016/j.aim.2008.03.007

Kuz10 A. Kuznetsov, Derived categories of cubic fourfolds, in Cohomological and geometric approaches to rationality problems, 219-243, Progr. Math. 282, Birkhäuser Boston, Boston, 2010. http: //dx.doi.org/10.1007/978-0-8176-4934-0_9

Kuz12 A. Kuznetsov, Instanton bundles on Fano threefolds, Cent. Eur. J. Math. 10 (2012), 1198-1231. http://dx.doi .org/10.2478/s11533-012-0055-1

LN13 M. Lahoz and J.C. Naranjo, Theta-duality on Prym varieties and a Torelli theorem, Trans. Amer. Math. Soc. 365 (2013), no. 10, 5051-5069. http://dx.doi.org/10.1090/ S0002-9947-2013-05675-9

Lie06 M. Lieblich, Moduli of complexes on a proper morphism, J. Alg. Geom. 15 (2006), 175-206. http://dx.doi.org/10.1090/S1056-3911-05-00418-2

Lie07 M. Lieblich, Moduli of twisted sheaves, Duke Math. J. 138 (2007), 23-118. http://dx.doi. org/10.1215/S0012-7094-07-13812-2

Luo93 Z. Luo, Factorization of birational morphisms of regular schemes, Math. Z. 212 (1993), 505509. http://dx.doi.org/10.1007/BF02571670

MS12 E. Macrì and P. Stellari, Fano varieties of cubic fourfolds containing a plane, Math. Ann. 354 (2012), 1147-1176. http://dx.doi.org/10.1007/s00208-011-0776-7

Mad00 C. Madonna, Rank-two vector bundles on general quartic hypersurfaces in $\mathbb{P}^{4}$, Rev. Mat. Complut. 13 (2000), 287-301. http://dx.doi.org/10.5209/rev_REMA.2000.v13.n2.17073

Mad05 C. Madonna, Rank 4 vector bundles on the quintic threefold, Cent. Eur. J. Math. 3 (2005), 404-411. http://dx.doi.org/10.2478/BF02475915

Mil80 J.S. Milne, Étale Cohomology, Princeton Math. Ser. 33, Princeton Univ. Press (1980).

Mir10 R.M. Miró-Roig and J. Pons-Llopis, $N$-dimensional Fano varieties of wild representation type, arXiv:1011. 3704.

MT01 D. Markushevich and A. S. Tikhomirov, The Abel-Jacobi map of a moduli component of vector bundles on the cubic threefold, J. Alg. Geom. 10 (2001), 37-62. 


\section{ACM BUNDLES ON CUBIC THREEFOLDS}

Orl93 D. Orlov, Projective bundles, monoidal transformations, and derived categories of coherent sheaves, Russian Acad. Sci. Izv. Math. 41 (1993), 133-141. http://dx.doi.org/10.1070/ IM1993v041n01ABEH002182

Orl09 D. Orlov, Derived categories of coherent sheaves and triangulated categories of singularities in Algebra, arithmetic, and geometry: in honor of Yu.I. Manin, Vol. II, 503-531, Progr. Math., 270, Birkhäuser Boston, Inc., Boston (2009). http://dx.doi.org/10.1007/ 978-0-8176-4747-6_16

PLT09 J. Pons-Llopis and F. Tonini, ACM bundles on Del Pezzo surfaces, Matematiche 64 (2009), $177-211$.

Sim94 C. Simpson, Moduli of representations of the fundamental group of a smooth projective variety I, Publ. Math. Inst. Hautes Études Sci. 79 (1994), 47-129. http://dx.doi.org/10.1007/ BF02698887

Tod08 Y. Toda, Moduli stacks and invariants of semistable objects on K3 surfaces, Adv. Math. 217, 2736-2781 (2008). http://dx.doi.org/10.1016/j.aim.2007.11.010

Tod13 Y. Toda, Stability conditions and extremal contractions, Math. Ann. 357 (2013), 631-685. http://dx.doi.org/10.1007/s00208-013-0915-4

Yos90 Y. Yoshino, Cohen-Macaulay modules over Cohen-Macaulay rings, London Math. Soc. Lecture Note Ser. 146, Cambridge Univ. Press, Cambridge, 1990. http://dx.doi.org/10.1017/ CB09780511600685

Martí Lahoz marti.lahoz@imj-prg.fr

Institut de Mathématiques de Jussieu - Paris Rive Gauche (UMR 7586), Université Pierre

Diderot / Université Pierre et Marie Curie, 5 rue Thomas-Mann, 75205 Paris cedex 13, France

Emanuele Macrì e.macri@neu.edu

Department of Mathematics, The Ohio State University, 231 W 18th Avenue, Columbus, OH 43210, USA

Current address: Department of Mathematics, Northeastern University, 360 Huntington Avenue, Boston, MA 02115, USA

Paolo Stellari paolo.stellari@unimi.it

Dipartimento di Matematica "F. Enriques", Università degli Studi di Milano, Via Cesare Saldini 50, 20133 Milano, Italy 